The Texas Medical Center Library

DigitalCommons@TMC

The University of Texas MD Anderson Cancer Center UTHealth Graduate School of Biomedical Sciences Dissertations and Theses (Open Access)
The University of Texas MD Anderson Cancer

Center UTHealth Graduate School of

Biomedical Sciences

$12-2020$

\title{
COMBINED INHIBITION OF DDR1 AND CDK4/6 INDUCES \\ SYNERGISTIC EFFECTS IN ER-POSITIVE, HER2-NEGATIVE BREAST CANCER WITH PIK3CA/AKT1 MUTATIONS
}

maryam shariati

maryam shariati

Follow this and additional works at: https://digitalcommons.library.tmc.edu/utgsbs_dissertations

Part of the Translational Medical Research Commons

\section{Recommended Citation}

shariati, maryam and shariati, maryam, "COMBINED INHIBITION OF DDR1 AND CDK4/6 INDUCES

SYNERGISTIC EFFECTS IN ER-POSITIVE, HER2-NEGATIVE BREAST CANCER WITH PIK3CA/AKT1

MUTATIONS" (2020). The University of Texas MD Anderson Cancer Center UTHealth Graduate School of Biomedical Sciences Dissertations and Theses (Open Access). 1084.

https://digitalcommons.library.tmc.edu/utgsbs_dissertations/1084

This Dissertation (PhD) is brought to you for free and open access by the The University of Texas MD Anderson

Cancer Center UTHealth Graduate School of Biomedical

Sciences at DigitalCommons@TMC. It has been accepted for inclusion in The University of Texas MD Anderson Cancer Center UTHealth Graduate School of Biomedical Sciences Dissertations and Theses (Open Access) by an authorized administrator of DigitalCommons@TMC. For more information, please contact digitalcommons@library.tmc.edu.

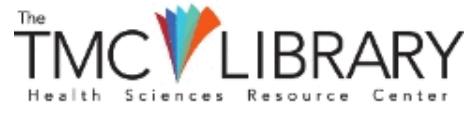




\section{COMBINED INHIBITION OF DDR1 AND CDK4/6 INDUCES SYNERGISTIC EFFECTS IN ER-POSITIVE, HER2-NEGATIVE BREAST CANCER WITH \\ PIK3CA/AKT1 MUTATIONS}

by

Maryam Shariati, M.S.

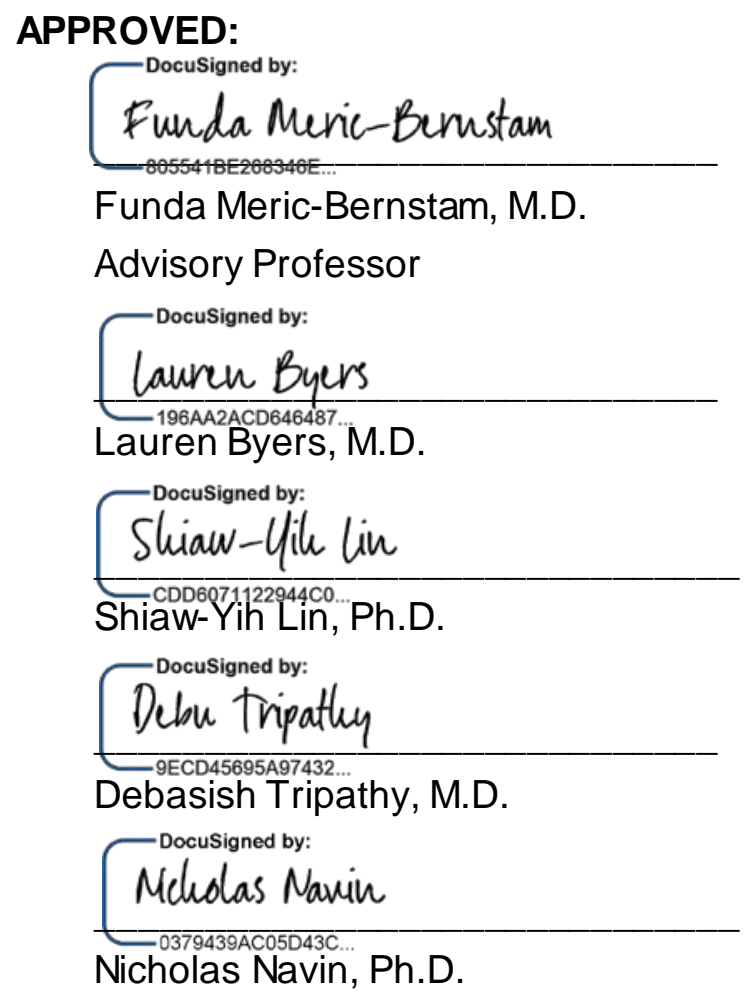

\section{APPROVED:}

Dean, The University of Texas

MD Anderson Cancer Center UTHealth Graduate School of Biomedical Sciences 


\title{
COMBINED INHIBITION OF DDR1 AND CDK4/6 INDUCES SYNERGISTIC EFFECTS IN ER-POSITIVE, HER2-NEGATIVE BREAST CANCER WITH \\ PIK3CA/AKT1 MUTATIONS
}

\author{
A \\ DISSERTATION \\ Presented to the Faculty of \\ The University of Texas \\ MD Anderson Cancer Center UTHealth \\ Graduate School of Biomedical Sciences \\ in Partial Fulfillment \\ of the Requirements \\ for the Degree of \\ DOCTOR OF PHILOSOPHY
}

by

Maryam Shariati, M.S.

Houston, Texas

December 2020 


\section{DEDICATION}

I am honored to dedicate this dissertation to my beloved parents, Mohammad Hassan Shariati and Nasrin Shirmohammadi, and my loving husband, Abdollah Orangi, whom I am blessed to have in my life.

To my kind and caring father who recently lost his life to lung cancer and whom I missed beyond comprehension. He was a knowledgeable and respectful person who lived a simple, yet prosperous life and taught me how to live with dignity. Sadly, I won't be able to share my achievement with him, but he left prideful and impactful fingerprints that enable me to carry on his legacy forever.

To my affectionate and exceptionally devoted mother whose love for me knows no bounds. An intelligent person who inspires me and ingrained within me the undeniable importance and value of hard work.

To my brilliant husband for his endless support and encouragement. For always standing by me and motivating me throughout the challenging times of completing this work. 


\section{ACKNOWLEDGMENTS}

It is with immense gratitude that I express the deepest appreciation to my advisor, Dr. Funda Meric-Bernstam, for giving me the opportunity to pursue this remarkable research under her impeccable instructions. She played a critical role in propelling my development by dedicating time, patience, and efforts to carrying out this project. Her persistent support, brilliant guidance, tremendous enthusiasm and knowledge have been essential to my achievement.

I extend my sincere gratefulness and acknowledgments to the members of my advisory committee: Dr. Lauren Byers, Dr. Shiaw-Yih Lin, Dr. Nicholas Navin and Dr. Debu Tripathy for their time, insightful suggestions, and interest in serving on my committee.

I would like to thank the talented members of Meric's lab for sharing their ideas and assistance during the time that I had the pleasure to work with them. In addition a thank you to Susanna Brisendine for her excellent administrative assistance.

I thank Visual Art at the University of Texas, MD Anderson Cancer Center for assistance with the illustrations in Figure 3, Figure 5, and, Figure 6. 


\title{
COMBINED INHIBITION OF DDR1 AND CDK4/6 INDUCES SYNERGISTIC EFFECTS IN ER-POSITIVE, HER2-NEGATIVE BREAST CANCER WITH PIK3CA/AKT1 MUTATIONS
}

\author{
Maryam Shariati, M.S. \\ Advisory Professor: Funda Meric-Bernstam, M.D.
}

Molecular alterations in the phosphatidylinositol 3-kinase (PI3K)/ serine/threonine protein kinase B (AKT) signaling pathway occur frequently in estrogen receptor-positive (ER-positive) breast tumors. Patients with ER-positive, human epidermal growth factor receptor 2-negative (HER2-negative) metastatic breast cancer are often treated with cyclin-dependent kinase (CDK4/6) inhibitors such as palbociclib in combination with endocrine therapy. Although this is a very effective regimen, disease progression ultimately occurs in most patients. Further, the modulators of palbociclib sensitivity remain unclear. The purpose of this study was to identify synthetic lethality partners that can enhance palbociclib's antitumor efficacy in ER-positive breast cancer with PI3K pathway alterations.

Using ER-positive isogenic breast cancer cell lines, we demonstrated that the efficacy of palbociclib is attenuated in the presence of PIK3CA or AKT1 mutations. Using a shRNA library screen targeting cancer-related human kinases, we identified that downregulation of discoidin domain receptor $1(D D R 1)$, a tyrosine kinase active in various cancers, is synthetically lethal with palbociclib. DDR1 knockdown by shRNA markedly reduced the growth of PIK3CA and AKT1 mutant and wild-type (WT) ER-positive cell lines in vitro. In addition, DDR1 pharmacological inhibitor, 7rh 
benzamide, decreased cell growth and inhibited cell cycle progression in all cell lines, while selectively enhancing the sensitivity of PIK3CA and AKT1 mutant cells to palbociclib. Combined treatment of palbociclib and 7rh further induced cell cycle arrest, decreasing PLK1, cyclin E2, and RB phosphorylation in PIK3CA and AKT1 mutant cell lines. In vivo, 7rh significantly enhanced the antitumor efficacy of palbociclib. Similarly, reverse phase protein array (RPPA) analysis of the xenografts revealed a greater PLK1 reduction in a combined 7rh and palbociclib treatment group. Our data indicate that DDR1 inhibition can augment the cell cycle suppressive effect of palbociclib and could be an effective targeted therapy for ERpositive, HER2-negative breast cancers with PI3K pathway activation. 


\section{TABLE OF CONTENTS}

APPROVAL PAGE

TITLE PAGE

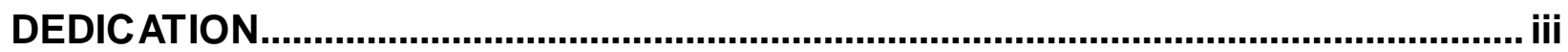

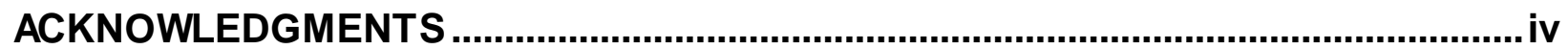

ABSTRACT

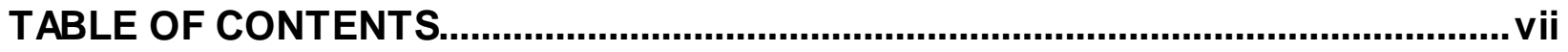

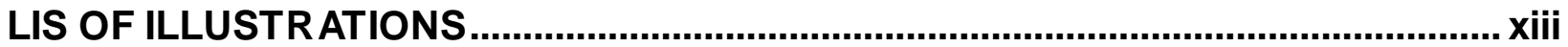

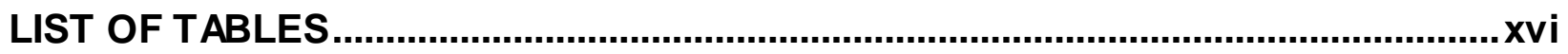

CHAPTER 1: INTRODUCTION

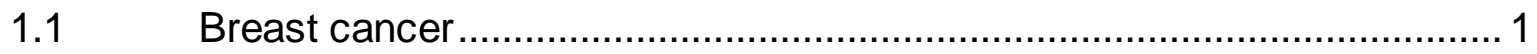

1.1.1 Breast cancer epidemiology ......................................................... 1

1.1.2 Histological classifications of breast cancer ........................................ 1

1.1.3 Molecular classifications of breast cancer ............................................ 3

1.2 Current therapies for ER-positive, HER2-negative advanced breast cancer 5

1.2.1 Role of ER and estrogen in ER-positive breast cancer ........................ 5

1.2.2 Selective estrogen receptor modulators ........................................... 6

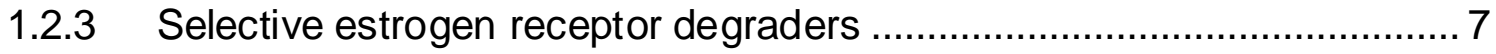

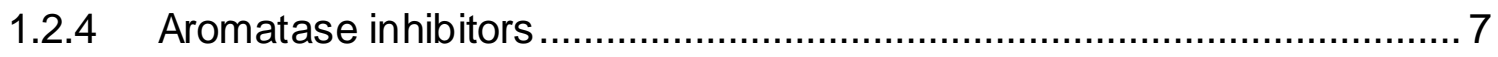

1.3 The $\mathrm{PI}$ IKK/AKT/mTOR pathway in breast cancer ................................ 9

1.3.1 Biological function of $\mathrm{PISK} / \mathrm{AKT}$ /mTOR pathway .................................. 9

1.3.2 Role of PI3K/AKT/mTOR signaling in carcinogenesis......................... 13 
1.3.3 Targeting PI3K and AKT1 in ER-positive breast cancer ...................... 16

1.4 Cell cycle in breast cancer ......................................................... 17

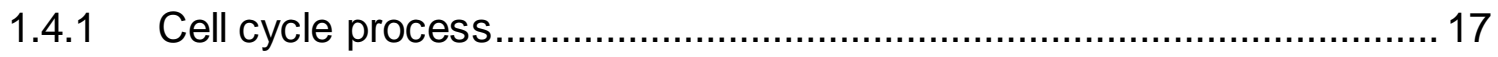

1.4.2 The regulation of cell cycle in breast cancer .................................... 18

1.4.3 Role of CDK 4/6 inhibitors in breast cancer ...................................... 19

1.5 Approved CDK 4/6 inhibitors for ER-positive, HER2-negative breast

cancer treatment

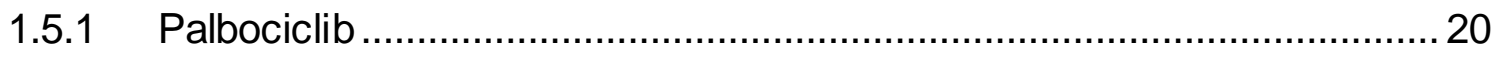

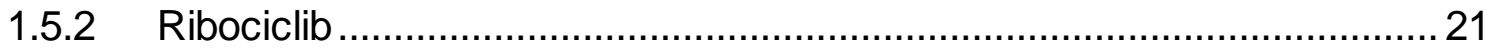

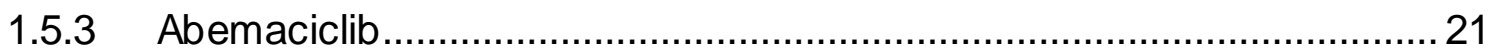

1.5.4 Mechanism of resistance to CDK 4/6 inhibitors in breast cancer .......... 23

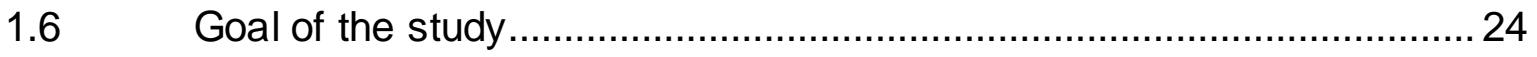

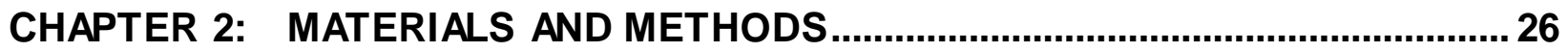

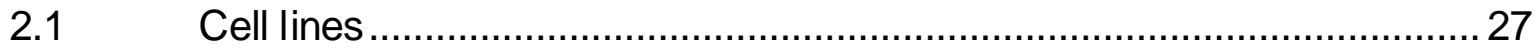

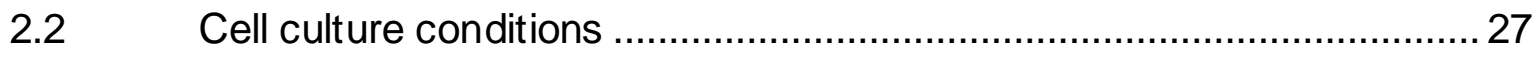

2.3 Drug cytotoxicity and proliferation assay ....................................... 28

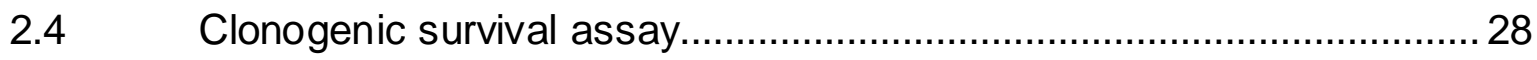

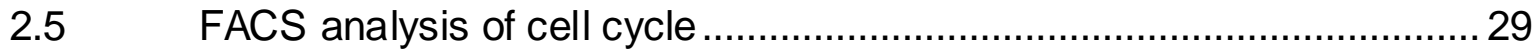

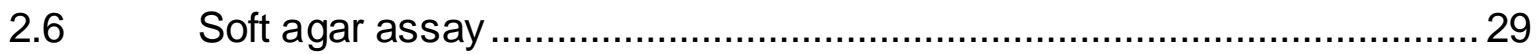

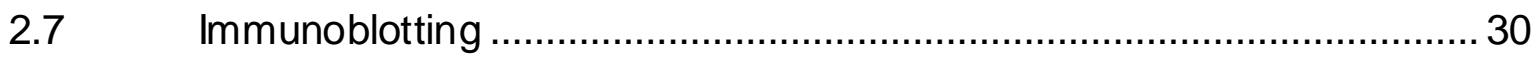

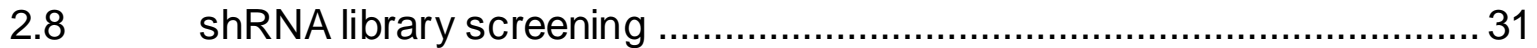

2.9 shRNA gene silencing and plasmid overexpression ........................... 34

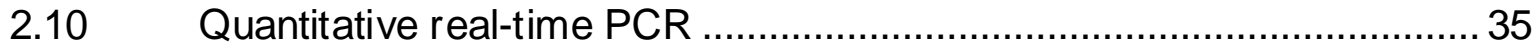


2.11 Reverse phase protein array (RPPA) analysis ................................. 36

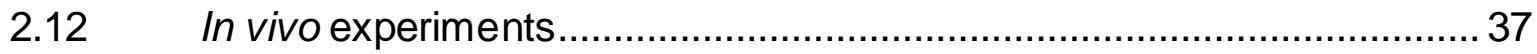

2.13 Immunohistochemical staining of human xenografts .......................... 38

2.14 Immunohistochemical scoring and imaging ........................................39

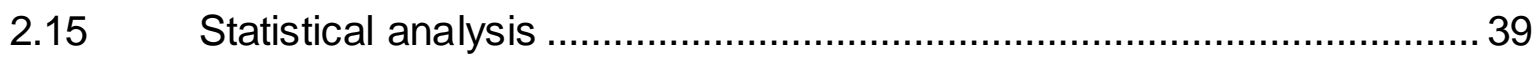

CHAPTER 3: PIK3CA AND AKT1 MUTATIONS ENHANCE BREAST CANCER

CELL GROWTH AND REDUCE PALBOCICLIB SENSITIVITY IN VITRO ................... 41

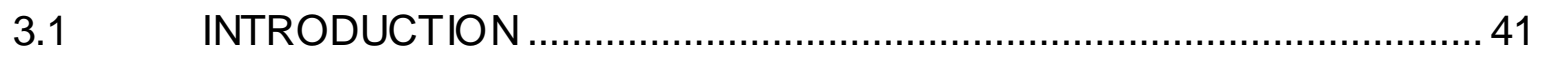

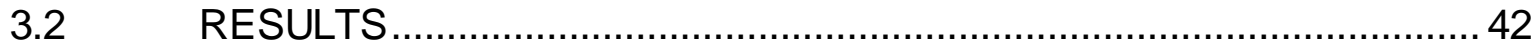

3.2.1 Validation and authentication of MCF7 isogenic cell lines ................... 42

3.2.2 Growth characterizations and effects of PIK3CA and AKT1 mutations in

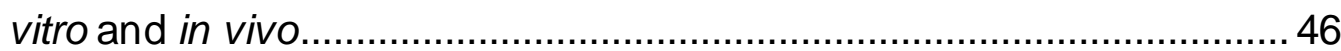

3.2.3 PIK3CA and AKT1 mutations reduce the sensitivity of palbociclib in vitro 49

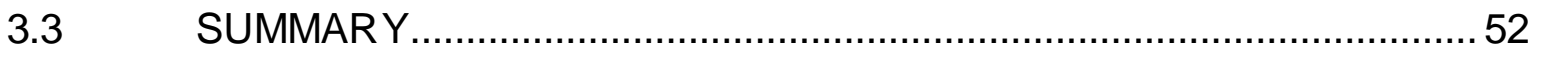

CHAPTER 4: SYNTHETIC LETHALITY SCREENING IDENTIFIED DDR1 AS SYNTHETIC LETHAL PARTNER OF PALBOCICLIB IN PIK3CA AND AKT1 MUTANT

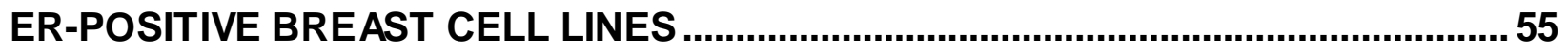

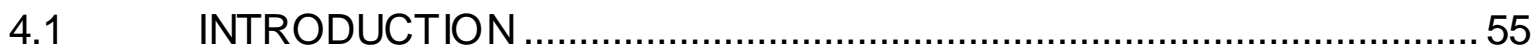

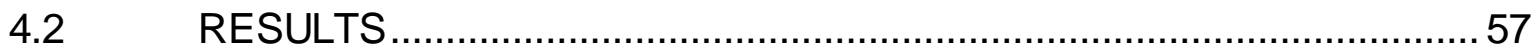

4.2.1 Using a pooled shRNA library and dropout selection screening to identify potential synthetic lethal genes in response to palbociclib........5 57 
4.2.2 Synthetic lethality screening identifies $D D R 1$ as a synthetic lethal partner of palbociclib in PIK3CA and AKT1 mutant ER-positive breast cancer cell lines 64

4.2.3 Correlation between DDR1 expression and ER status in a cohort of breast cancer patients

4.3 SUMMARY 69

CHAPTER 5: DDR1 SUPPRESSION INHIBITED PROLIFERATION AND

SELECTIVELY SENSITIZED PIK3CANAKT1 MUTANT CELLS TO PALBOCICLIB

TREATMENT 70

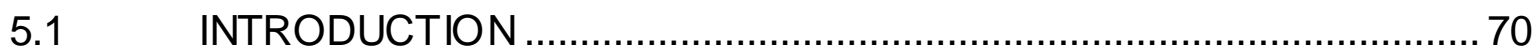

5.1.1 Structure, activation, and function of DDR1 ….................................. 70

5.1.2 DDR1 as a target in breast cancer ................................................ 73

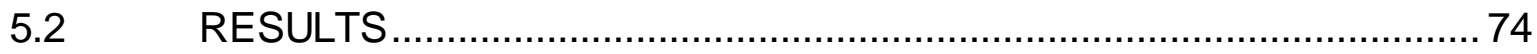

5.2.1 Silencing DDR1 with different targeted shRNAs, and validating knockdown efficiency

5.2.2 DDR1 suppression inhibits proliferation and selectively sensitizes PIK3CAVAKT1 mutant cells to palbociclib treatment 76

5.3 SUMMARY 80

CHAPTER 6: DDR1 INHIBITION WITH 7RH SENSITIZED PIK3CA AND AKT1 MUT ANT CELLS TO PALBOCICLIB TREATMENT BY INDUCING CELL CYCLE ARREST $\quad 82$

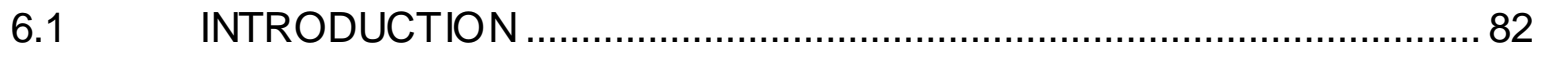

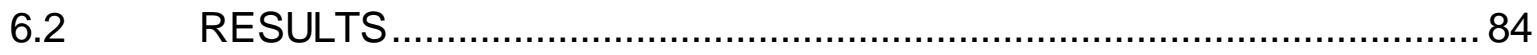


6.2.1 DDR1 and CDK4/6 co-inhibition induces a synergistic response and anti-proliferation effect in PIK3CA and AKT1 mutant cells

6.2.2 DDR1 inhibition enhances palbociclib-induced cell cycle arrest of PIK3CA and AKT1 mutant cells

6.2.3 DDR1 inhibition enhances palbociclib-induced cell cycle arrest of ERpositive breast cancer cell lines with PIK3CA mutations.

6.3 SUMMARY

CHAPTER 7: PHENOTYPIC CHANGES FOLLOWING DIFFERENTIAL DDR1

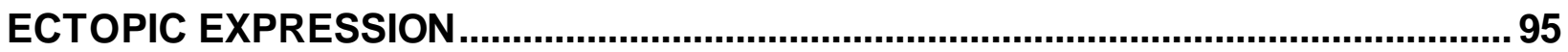

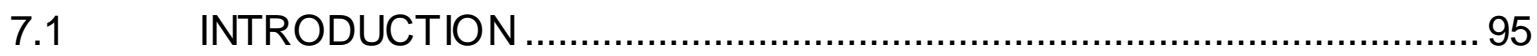

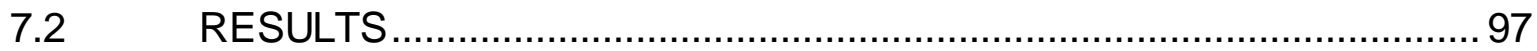

7.2.1 Phenotypic changes following ectopic expression of DDR1 in MCF10A benign breast epithelial cells

7.2.2 Functional impact of reported DDR1 mutations in MCF10A benign breast epithelial cells 100

7.2.3 Sensitivity to DDR1 inhibitor 7rh in a panel of breast cancer cell lines with different ER statuses and PI3K pathway mutations

7.3 SUMMARY 104

CHAPTER 8: CHAPTER 8: DDR1 INHIBITOR 7RH INHIBITED THE GROWTH OF PIK3CA XENOGRAFT TUMORS AND SYNERGIZED WITH PALBOCICLIB IN VIVO 106

8.1 INTRODUCTION 106

8.2 RESULTS 107 
8.2.1 DDR1 inhibitor 7rh inhibits the growth of PIK3CA xenograft tumors and

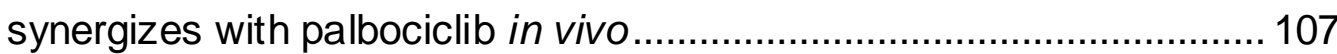

8.2.2 DDR1 inhibition induces cell cycle arrest and synergizes with palbociclib to suppress tumor growth in vivo 109

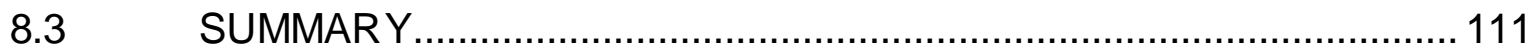

CHAPTER 9: CHAPTER 9: DISCUSSION AND FUTURE DIRECTIONS ................. 113

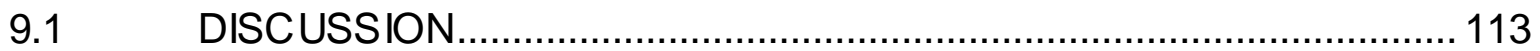

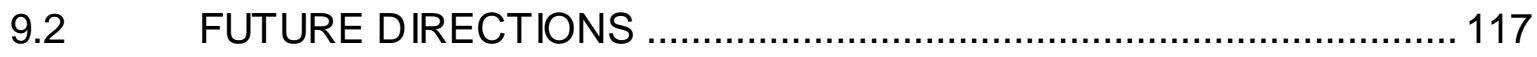

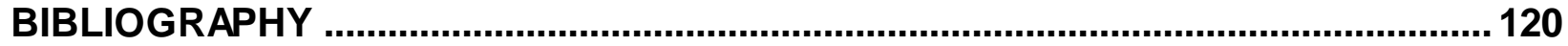

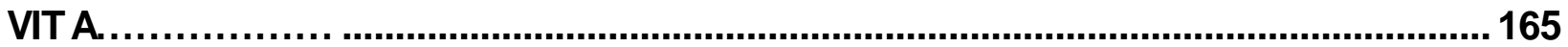




\section{LIST OF ILLUSTRATIONS}

Figure 1. Histological classification of breast cancer. .............................................. 2

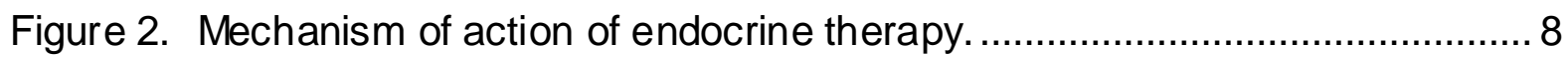

Figure 3. Representation of PI3K/AKT/mTOR signaling pathway. ........................ 11

Figure 4. Structural domains of PIK3CA and AKT1 with hotspot mutations............. 14

Figure 5. Function of CDK4/6 in cell cycle progression. ........................................ 19

Figure 6. CDK4/6 inhibitors and the upstream mitogenic forces inducing cyclin D1

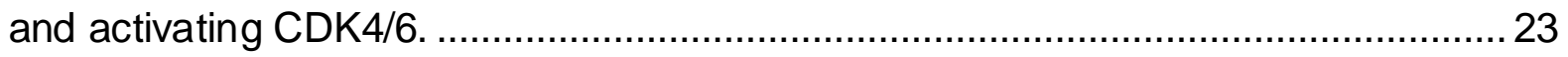

Figure 7. MOI and viral integration calculated based on Poisson distribution........... 33

Figure 8. Validation of PIK3CA and AKT1 alterations in MCF7 isogenic cell lines... 45

Figure 9. PIK3CA and AKT1 mutations confer growth advantage in vitro and in vivo.

Figure 10. PI3K pathway signaling and colony formation ability in PIK3CA and AKT1 mutations. 48

Figure 11. PIK3CA and AKT1 mutations show less sensitivity to palbociclib............50

Figure 12. PIK3CA and AKT1 mutations modulate RB phosphorylation and cell cycle

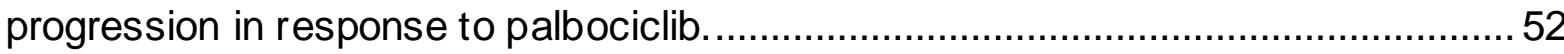

Figure 13. Transduction of MCF7 cell lines with lentiviral shRNA library and negative selection screening 60

Figure 14. shRNA library screening identifies DDR1 as a candidate synthetic lethal partner for palbociclib in both PIK3CA and AKT1 mutant cell lines 65 Figure 15. The correlation between DDR1 expression and ER status, in a cohort of breast cancer patients using TCGA and METABRIC dataset. 
Figure 16. The expression of DDR1 in different breast cancer subtypes and ERpositive group with PIK3CAAKT1 mutations using TCGA and MRTABRIC dataset.

Figure 17. Structural domains of five different DDR1 isoforms.

Figure 18. DDR1 protein and mRNA level in shRNA-induced DDR1 silencing cell lines.

Figure 19. Silencing DDR1 sensitizes PIK3CA and AKT1 mutant cells to palbociclib

treatment. 79

Figure 20. DDR1 and CDK4/6 co-inhibition induces more sensitivity and antiproliferation effect in PIK3CA and AKT1 mutant cells.

Figure 21. DDR1 inhibition enhances palbociclib-induced cell cycle arrest of PIK3CA and AKT1 mutant cells

Figure 22. The effect of DDR1 and CDK4/6 co-inhibition in ER-positive PIK3CAmutated breast cancer cell lines.

Figure 23. Mutation profile and frequency plot for DDR1 transcript.

Figure 24. Phenotypic changes following ectopic expression of DDR1 in MCF10A benign breast epithelial cells.

Figure 25. Functional impact of reported DDR1 mutations in MCF10A benign breast epithelial cells. 102

Figure 26. Sensitivity to DDR1 inhibitor 7rh in a panel of breast cancer cell lines with different ER statuses and PI3K pathway mutations. 103 Figure 27. Dual inhibition of DDR1 and CDK4/6 decreases the tumor growth of PIK3CA-mutated cells in vivo 109 
Figure 28. DDR1 inhibition induces cell cycle arrest and synergizes with palbociclib

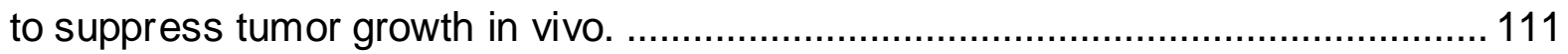




\section{LIST OF TABLES}

Table 1. Molecular classification of breast cancer.

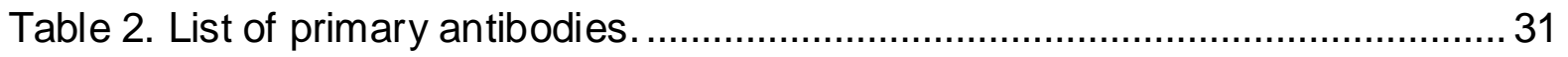

Table 3. DNA fingerprinting of the isogenic MCF7 cell lines. ................................ 43

Table 4. List of the candidate genes that confer a selective disadvantage in the

presence of palbociclib.

Table 5. List of the selected potential synthetic lethal genes for palbociclib treatment.

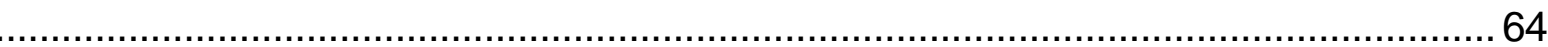

Table 6. FDA-approved non-selective oral DDR1 and DDR2 inhibitors................... 83

Table 7. Reported selective and non-selective DDR1 and DDR2 inhibitors. ............ 84 


\section{CHAPTER 1: INTRODUCTION}

\subsection{Breast cancer}

\subsubsection{Breast cancer epidemiology}

Breast cancer represents $14 \%$ of all new cancer cases in the Unites States, and approximately 1 in 8 women will develop invasive breast cancer over the course of her lifetime [1]. It is the leading diagnosed cancer type representing $29 \%$ of all cases, and is the second-most-common cause of cancer death in women [2]. Recent advances in health care and medical science have accelerated our ability to detect the disease in its early stages and have decreased the rate of progression into a metastatic state. Nonetheless, the increasing incidence of breast cancer indicates the urgent need for developing more advanced treatments and therapeutic approaches that can address the molecular mechanisms underlying the disease's aggressiveness, so as to reduce symptom burden and mortality in patients with breast cancer.

\subsubsection{Histological classifications of breast cancer}

Breast cancer is a heterogeneous disease classified into different types based on the specific cells affected, tumor morphology, aggressiveness, and the intrinsic molecular expression [3]. Traditionally, breast cancer is divided into ductal carcinomas in situ that originate in the milk ducts, and lobular carcinomas in situ that form in the milk gland (Figure 1) [4,5]. Both ductal and lobular carcinomas can be non-invasive, meaning the cancer cells have not spread into surrounding tissue, or invasive, meaning the disease cells have disseminated into nearby tissues or distant 
organs [6]. Invasive ductal carcinoma is the most frequent type (40\%-75\% of cases [4].

All breast carcinomas are histologically graded according to the Nottingham histological grading system which combines tumor size $(\mathrm{T})$, lymph node invasion $(\mathrm{N})$, and spread into distant metastatic sites (M) [7, 8]. In this system, numbers are used for each stage to describe the size and location of the tumor (0-3), the degree of lymph node invasion (0-3), and presence of distant metastasis (0-1). The breast cancer stages are determined by combining TNM classification along with their numerical grades, where stage 0 indicates a non-invasive tumor and stages $>0$ correspond to invasive cancer.

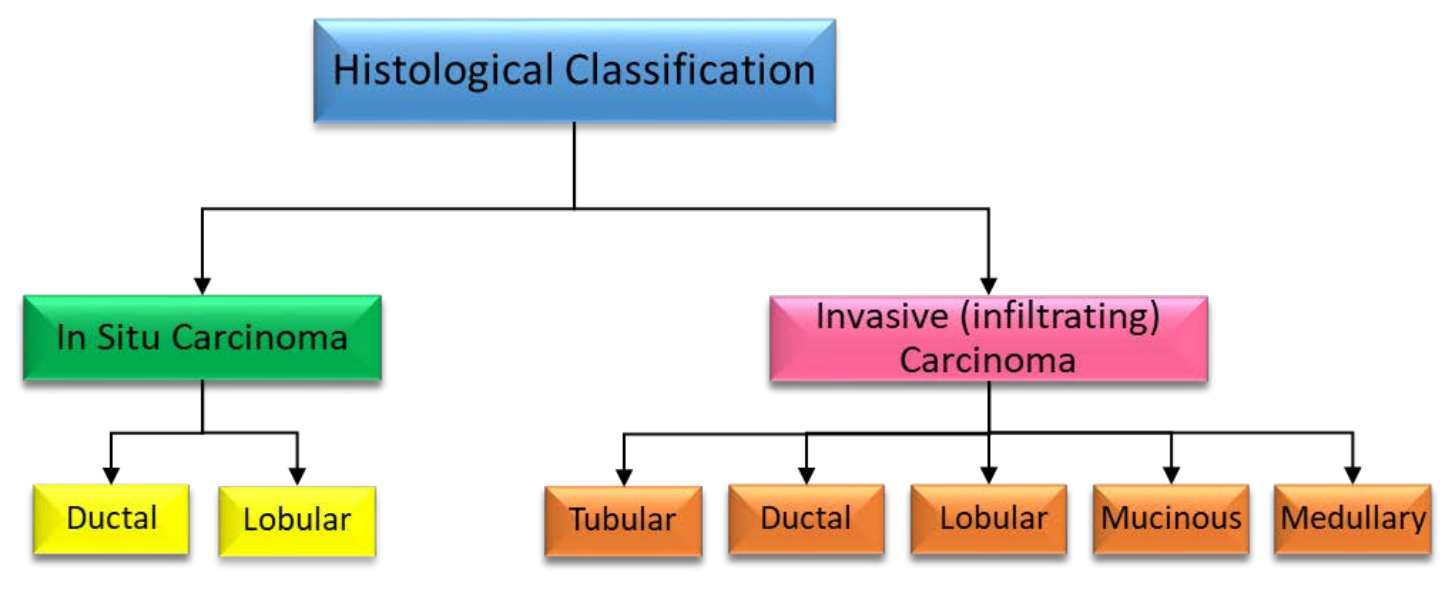

Figure 1. Histological classification of breast cancer.Figure showing categorization of breast cancer based on architectural features and growth patterns. Breast cancer is classified into in situ carcinoma and invasive (infiltrating) carcinoma. 


\subsubsection{Molecular classifications of breast cancer}

Over the past decades, gene expression profiling and next-generation sequencing have been used to clarify breast cancer classification and to advance predictive prognosis and gnomically-informed therapeutic approaches [9]. In breast cancer research, the focus is usually on the molecular profile characteristics, which can guide in planning treatment and developing new targeted therapies.

At the molecular level breast cancers are classified into different subtypes based on the expression of hormonal receptors, including estrogen receptor (ER), progesterone receptor (PR), and human epidermal growth factor receptor 2 (HER2) (Table 1) [10]. ER and PR, which are expressed in approximately $75 \%$ of breast cancers, are nuclear receptors that stimulate breast epithelium growth [11]. Luminal tumors that start in the luminal lining of the mammary ducts are mostly ER-positive and are categorized as luminal A or B. Luminal A tumors are low-grade, ER/PRpositive, HER2-negative with a low Ki67 score, whereas luminal B tumors are highgrade, ER-positive, PR-positive/negative, HER2-positive/negative with a high Ki67 score [12]. Most luminal A tumors are low-grade and are therefore associated with better survival rates. The clinical management of these groups includes endocrine therapy through ER antagonism (tamoxifen, fulvestrant) and estrogen production suppression (aromatase inhibitors) [13, 14]. 


\begin{tabular}{|c|c|c|c|c|c|c|}
\hline $\begin{array}{l}\text { Molecular } \\
\text { Subtype }\end{array}$ & $\begin{array}{l}\text { ER/PR/HER2 } \\
\text { Status }\end{array}$ & $\begin{array}{l}\text { Proliferation } \\
\text { Index (Ki67) }\end{array}$ & $\begin{array}{l}\text { Histological } \\
\text { Grade }\end{array}$ & Prognosis & Prevalence & $\begin{array}{l}\text { Treatment } \\
\text { Vulnerability }\end{array}$ \\
\hline & $\mathrm{ER}+$ & & & & & \\
\hline Luminal A & $\begin{array}{l}\text { PR+ } \\
\text { HER2- }\end{array}$ & Low & Low & Good & 50-60\% & Endocrine therapy \\
\hline Luminal B & $\begin{array}{l}\text { ER+ } \\
\text { PR+/- } \\
\text { HER2+/- }\end{array}$ & High & $\begin{array}{l}\text { Intermediate } \\
\text { High }\end{array}$ & Intermediate & 15-20\% & $\begin{array}{l}\text { Endocrine therapy/ } \\
\text { Cytotoxic } \\
\text { chemotherapy }\end{array}$ \\
\hline $\begin{array}{l}\text { HER2- } \\
\text { enriched }\end{array}$ & $\begin{array}{l}\text { ER- } \\
\text { PR- } \\
\text { HER2+ }\end{array}$ & High & High & Poor & 10-15\% & $\begin{array}{l}\text { HER2 blockade } \\
\text { (Trastuzumab) }\end{array}$ \\
\hline Basal-like & $\begin{array}{l}\text { ER- } \\
\text { PR- } \\
\text { HER2- }\end{array}$ & High & High & Poor & 15\% & $\begin{array}{l}\text { Cytotoxic } \\
\text { chemotherapy }\end{array}$ \\
\hline Claudin-low & $\begin{array}{l}\text { ER- } \\
\text { PR- } \\
\text { HER2- }\end{array}$ & Low & $\begin{array}{l}\text { Intermediate } \\
\text { High }\end{array}$ & Poor & 10\% & $\begin{array}{l}\text { Cytotoxic } \\
\text { chemotherapy }\end{array}$ \\
\hline Normal-like & $\begin{array}{l}\text { ER+ } \\
\text { PR+/- } \\
\text { HER2- }\end{array}$ & Low & Low/High & Intermediate & 7.8\% & Undetermined \\
\hline
\end{tabular}

Table 1. Molecular classification of breast cancer.Table showing different subtypes of breast cancer and their characteristics based on hormonal receptors expression.

The hormone receptor-negative (ER/PR-negative) breast cancers with HER2 amplification comprise the HER2 subtype. HER2-positive tumors have aggressive clinical behavior and poor prognosis, but are responsive to HER-2-targeted treatments [15]. On the other hand, approximately $15 \%$ of breast tumors are basallike triple-negative breast cancers (TNBC) that do not express ER, PR, or HER2 and are often associated with an aggressive phenotype with poor clinical outcomes [16, 17].

Another breast cancer subtype is claudin-low tumors, which are triplenegative (ER/PR/HER2-negative) with an intermediate survival rate that lies between those of the basal-like and luminal groups. A hallmark of claudin-low tumors is the low expression of luminal and cell adhesion markers including claudin 
3,4 , and 7 and E-cadherin [18]. Tumors of this subtype are enriched in epithelialmesenchymal transition markers and cancer stem cell-like signatures [19]. The normal-like breast cancer subtype accounts for $7.8 \%$ of all breast cancer and shares a similar immunohistochemical status to luminal A group (ER-positive, PRpositive/negative, HER2-negative) [20]. The subtype shows a varying gene expression profile that includes adipose and stromal genes as well as basal epithelial genes [21].

Comprehensive analysis of breast cancer mutational landscape by using next-generation sequencing technologies has provided further information and expanded the molecular classification. Such next-generation sequencing studies have identified several mutated driver genes in breast cancer including TP53, PIK3CA, PTEN, FGFR1, RB1, MYC and GATA3 [22, 23]. The most common mutations are PIK3CA, TP53, and GATA3, which have distinct pathological and clinical impacts that are differentially expressed across different subtypes [24]. PIK3CA mutations are dominant in luminal A and luminal B groups while TP53 alterations are more prevalent in basal-like breast cancers [11]. The HER2-positive, ER/PR-negative breast tumors are associated with mitogen-activated protein kinase (MAPK) pathway overactivation and epidermal growth factor receptor (EGFR)/HER2 signaling signature [25].

\subsection{Current therapies for ER-positive, HER2-negative advanced breast cancer}

\subsubsection{Role of ER and estrogen in ER-positive breast cancer}

The ER-positive, HER2-negative luminal breast cancer is the mostcommon subtype in women of all racial and ethnic groups and is highly heterogeneous [2]. ER 
is a transcription factor that regulates the expression of various genes involved in cell division and plays an important role in the growth and development of mammary glands [26]. ER $\alpha$ and ER $\beta$ members of the nuclear receptor family are the two types of mammalian ERs that have been identified with ERa being the predominant regulator in ER-positive breast cancer, conversely, the role of ER $\beta$ is not well known $[27,28]$. Upon binding of $17 \beta$-estradiol (E2) to ER, the receptor is activated and binds to specific DNA sequences known as the estrogen response element to drive the transcription of target genes $[29,30]$. Various transcription factors, coactivators and genes regulate the genomic function of ER, such as estrogen receptor 1 (ESR1), GATA3, and FoxA1 [31, 32].

Advanced breast cancer, which includes metastatic and locally advanced tumors, has a poor prognosis, with the 5 years survival rate of approximately $26.9 \%$ [33]. Most metastatic breast cancer is HR-positive (75 \%) and HER2-negative (70\%) [34]. Endocrine therapy is usually the first-line treatment option for these patients and includes selective estrogen receptor modulators (SERMs), selective estrogen receptor degraders (SERDs), and aromatase inhibitors (Als) (Figure 2) [35].

\subsubsection{Selective estrogen receptor modulators}

Similar to estrogen, SERMs act as ligands binding to ER but do not induce conformational change or activation of the receptor; thus, they function as agonists or antagonists to block the expression of ER-induced genes [36-38]. In randomized clinical trials, SERMs were well tolerated with good efficacy and safety [39]. Tamoxifen, a nonsteroidal compound, was the first SERM approved by the US Food and Drug Administration (FDA) for the treatment of ERa breast cancer [40], and is 
routinely used to treat all stages of ER-positive breast cancer. Phase III trials of tamoxifen have shown a $31 \%$ to $69 \%$ reduction in breast cancer incidence $[41,42]$. However, despite its clinical efficacy, long-term exposure to tamoxifen was found to increase the risk for developing endometrial cancer $[43,44]$. Moreover, expanded exposure to SERMs is associated with therapeutic resistance due to multiple causes, such as loss of ER expression and acquired ESR1 mutations [45-47]

\subsubsection{Selective estrogen receptor degraders}

In contrast to SERMs, SERDs bind to ERs and function by inhibiting the receptor dimerization, thereby inducing its proteasome-dependent degradation and ultimately abolishing the ER pathway activity [48]. Fulvestrant is a SERD with an antagonist effect that was initially approved as a single-agent, first-line therapy in postmenopausal patients with HR-positive advanced breast cancer $[49,50]$. Preclinical data showed that patients with resistance to tamoxifen respond to fulvestrant treatment and that fulvestrant induced growth inhibition in tamoxifenresistant tumors $[51,52]$.

\subsubsection{Aromatase inhibitors}

Aromatase inhibitors (Als) are anti-estrogenic drugs that block the activity of the aromatase enzyme, which converts androgen to estrogen; by doing so, theses inhibitors reduce the level of free estrogen that stimulates breast cancer growth [53]. The two classes of Als approved for clinical use include type I steroidals (eg. exemestane) and type II nonsteroidals (eg. letrozole) [54]. Genetic polymorphisms in the aromatase gene, low-molecular weight cyclin E overexpression, or increased 
P44/42 MAPK may be responsible for Al therapy resistance in some patients with HR-positive, HER2-negative advanced breast cancer [55-57].

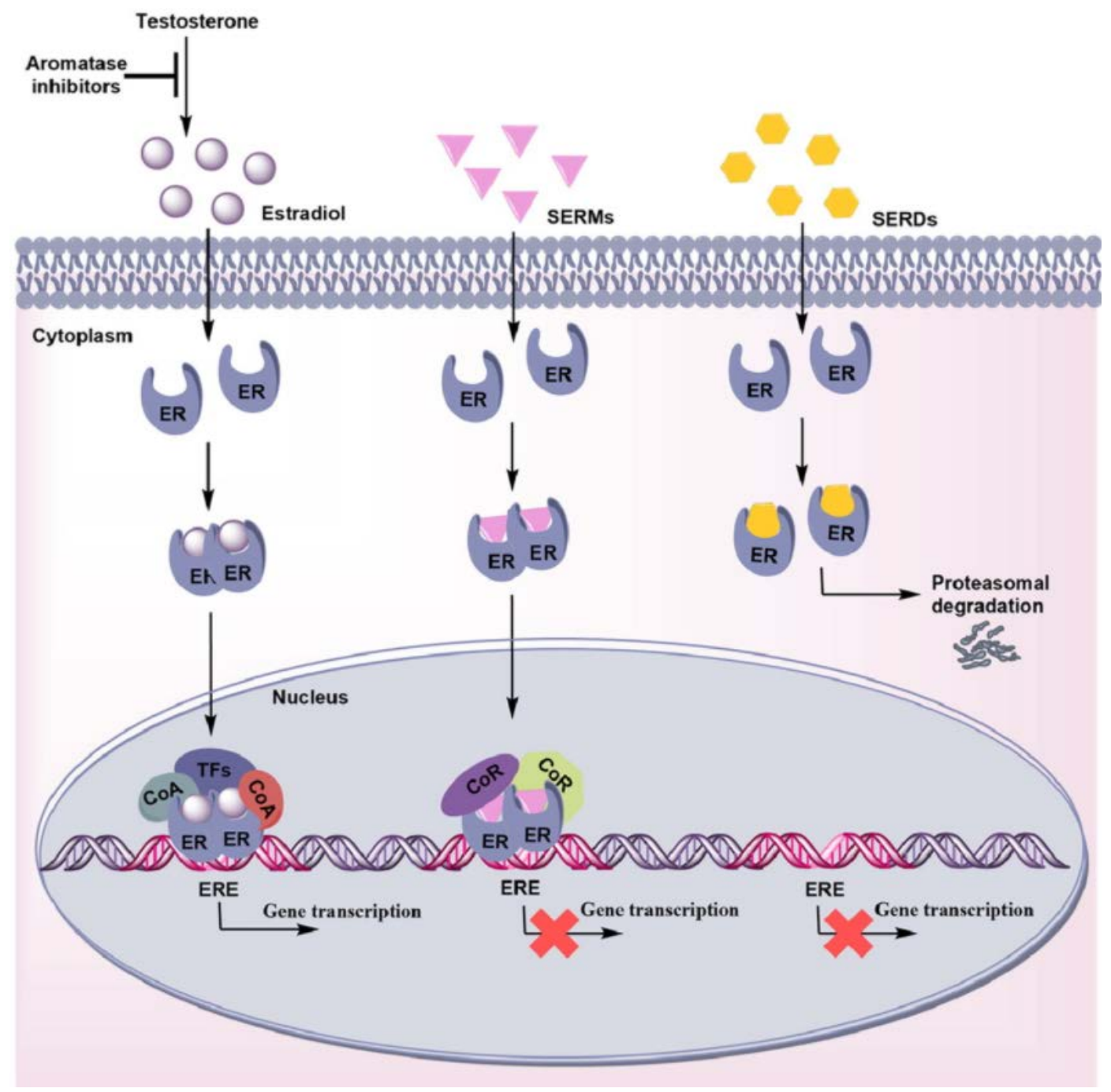

Figure 2. Mechanism of action of endocrine therapy. The effects of endocrine therapies (SERMs, SERDs and aromatase inhibitors) on the estrogen receptor pathway. SERMs prevent ER signaling by binding to ER and causing an inactive complex, SERDs prevent ER signaling by causing degradation of ER and aromatase inhibitors prevent ER signaling by inhibiting synthesis of estradiol (estrogen). (Adopted with permission from Patel, H.K. and T. Bihani, Selective estrogen receptor 
modulators (SERMs) and selective estrogen receptor degraders (SERDs) in cancer treatment. Pharmacology \& Therapeutics, 2018).

\subsection{The PI3KIAKT/mTOR pathway in breast cancer}

\subsubsection{Biological function of PI3KIAKT/mTOR pathway}

Breast cancer has a high degree of heterogeneity [58], with diverse clinical and histological varieties. Understanding the molecular signaling responsible for breast tumor heterogeneity is necessary for developing targeted therapeutic approaches. Phosphatidylinositol 3-kinases (PI3Ks) are a conserved family of lipid kinases divided into three classes based on their structures and specific lipid substrates. Class I PI3Ks are heterodimeric molecules that are further divided into class IA and class IB. Class IA comprises a p110 catalytic subunit containing three isoforms of $p 110 \alpha, p 110 \beta$ and $p 110 \delta$ and a p85 regulatory subunit with five variants of p85a, p55a, p50a, p85 $\beta, p 55 y$. Class IB PI3Ks are heterodimers containing a p110y catalytic subunit and a p101 regulatory subunit [59]. The physiological role of class II and III PI3Ks in signal transduction remains unclear.

In response to extracellular growth signals, receptors tyrosine kinase (RTK) binds to PI3K directly or indirectly through insulin receptor substrate (IRS). PI3K activation, which can also be induced by $\mathrm{G}$ protein-coupled receptor, recruits class I PI3K to plasma membrane, relieving p85 inhibition of p110 and resulting in phosphorylation of phosphatidylinositol (Ptdlns) 4,5-bisphosphate (PIP2) to generate Ptdlns (3,4,5)-triphosphate (PIP3) [59]. This triggers translocation of AKT, a serine/threonine protein kinase $\mathrm{B}$, to the membrane, where it binds to PIP3 through its pleckstrin homology $(\mathrm{PH})$ domain, allowing phosphoinositide-dependent kinase 1 
to access and phosphorylate AKT at Thr308. Full activation of AKT requires phosphorylation of Ser473, which can be catalyzed by PDK2, mechanistic target of rapamycin (mTOR) complex 2 (mTORC2), or DNA-dependent protein kinase (Figure 3).

Once phosphorylated, activated AKT translocates to various intracellular locations where it phosphorylates and modulates the function of numerous substrates, many of which are involved in cancer initiation and progression [60]. AKT activity is negatively regulated through dephosphorylation by the tumor suppressors phosphatase and tensin homolog (PTEN) and pleckstrin homology domain leucinerich-protein phosphatase (PHLPP) [61]. PTEN dephosphorylates the PIP3 component of PI3K back to PIP2, and PHLPP removes the phosphate group from AKT at the Ser473 residue [62].

Activated AKT phosphorylates a variety of protein substrates involved in survival and cellular growth. mTOR, the main downstream target of PI3K/AKT signaling, is a key regulator of cell metabolism that once activated phosphorylates ribosomal protein S6 kinase (p70S6K) and elF4E binding-protein-1 (4E-BP1) to promote protein synthesis. Tuberous sclerosis complex 1 and 2 (TSC1 and TSC2) tumor suppressors which are negative regulators of mTOR/S6K pathway, are phosphorylated by AKT, resulting in their inhibition $[63,64]$. In addition, AKT phosphorylates proline rich AKT substrate of $40 \mathrm{kDa}$ (PRAS40) at Thr246 and yesassociated protein (YAP) at Ser127 to induce their interactions with 14-3-3 which mediates nuclear export $[65,66]$. 
AKT exerts its effect on cell cycle progression by phosphorylating and inhibiting cyclin dependent kinase inhibitors p21 and p27, which function as G1 checkpoints to arrest cell cycle [64]. AKT regulates apoptosis through inhibition of BAD, BCL-2-like protein 11, caspase 9, and forkhead box O1 (FoxO1) [67-70]. It also phosphorylates mouse double-minute 2 homolog, allowing its entry into the nucleus, which results in p53 degradation [71-73] (Figure 3)

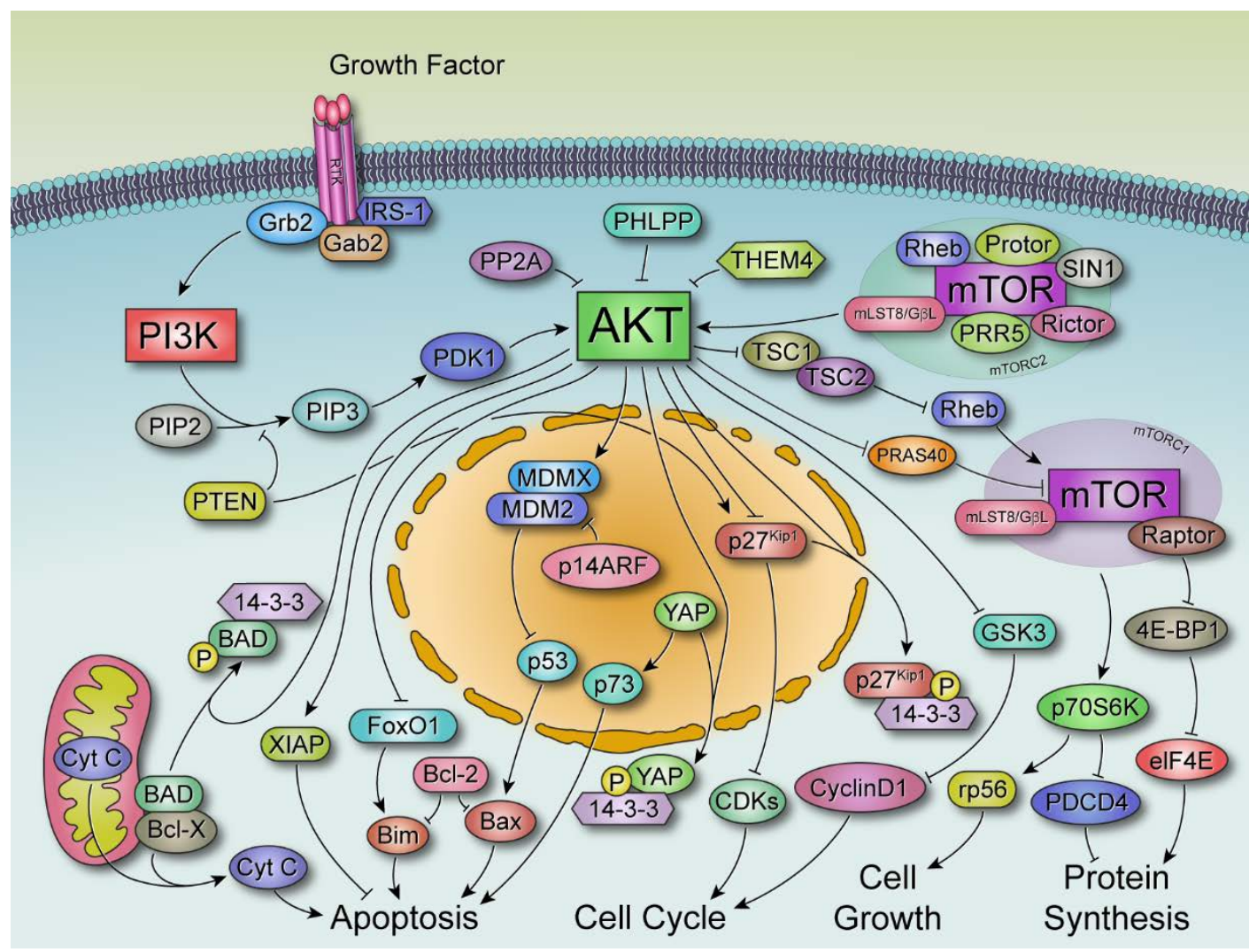

\section{Figure 3. Representation of PI3KIAKT/mTOR signaling pathway.}

Shown are the key molecular targets involved in AKT signaling. Upon growth factor binding to receptor tyrosine kinase (RTK), phosphoinositol bisphosphate (PIP2) is converted to phosphoinositol trisphosphate (PIP3), which recruits AKT and phosphoinositide-dependent kinase-1 (PDK1) to the plasma membrane by binding the $\mathrm{PH}$ domain to membrane lipids. PDK1 and mammalian target of rapamycin 
complex 2 (mTORC2) phosphorylate AKT, resulting in full activation of AKT. Phosphatase and tensin homolog (PTEN) and PH domain leucine-rich-protein phosphatase (PHLPP) negatively regulate AKT through dephosphorylation of PIP3 and AKT (serine-473 residue) respectively. Once activated AKT phosphorylates and inhibits the proline-rich AKT substrate of $40 \mathrm{kDa}$ (PRAS40) and tuberous sclerosis complex 1 and 2 (TSC1 and TSC2) to promote protein synthesis. AKT blocks apoptosis through inhibition of BCL-2-antagonist of cell death (BAD), BCL-2-like protein 11 (BIM), and forkhead box protein 01 (FoxO1). It also phosphorylates MDM2, resulting in p53 degradation. AKT prevents cell cycle arrest and cyclin D1 degradation by phosphorylating and inhibiting cyclin dependent kinase inhibitor p27, and glycogen synthase kinase $3 \beta$ (GSK3 $\beta$ ). (Adopted with permission from Shariati, $M$. and F. Meric-Bernstam, Targeting AKT for cancer therapy. Expert Opinion on Investigational Drugs, 2019. 28(11): p. 977-988.)

Mitogen signals such as insulin-like growth factor 1 (IGF1), activate the MAPK/extracellular-signal-regulated kinase (ERK) pathway, resulting in TSC1/TSC2 complex inhibition, thus stimulating mTOR complex 1 (mTORC1) [74]. In addition, the Wnt pathway, a major regulator of cell growth, is involved in mTORC1 activation through glycogen synthase kinase (GSK)-3 $\beta$ phosphorylation and subsequent TSC2 inhibition. The main negative regulator of the pathway is PTEN lipid phosphatase which converts PIP3 to PIP2 by removing the 3' phosphate from PIP3. The PISK/AKT pathway negatively controls PTEN at the transcriptional level through regulation of transcription factor nuclear factor-kB (NF-kB) and tumor necrosis factor a (TNFa), which in turn repress PTEN expression.

The pathway is also controlled by protein phosphatase $2 \mathrm{~A}$ and phosphatase PHLPP, which dephosphorylate AKT at Thr308 and Ser473, respectively. mTORC1 triggers the most prominent negative feedback regulation of PI3K signaling. During chronic insulin-mediated PI3K stimulation, mTORC1 and S6K activation can lead to 
downregulation of IRS-1 adopter protein through transcriptional regulation and proteasomal degradation [74]. In another negative feedback control mechanism, S6K1 is able to phosphorylate IRS-1 at multiple serine residues, preventing its binding to RTKs and initiation of PI3K signaling.

\subsubsection{Role of PI3KIAKT/mTOR signaling in carcinogenesis}

The PI3K/AKT/mTOR signaling network is an important pathway that integrates extracellular and environmental stimuli translating them into intracellular signals that drive cellular functions such as cell growth, proliferation, motility, survival, and vesicular trafficking. Aberrant activation of PI3K signaling contributes to most cancer hallmarks, including increased cell cycle progression, metabolism, survival, and motility. Molecular alterations of the major nodes of this signaling axis have been found in numerous tumor types.

PI3K activity is regulated by growth factor receptors; therefore, any modulation in RTKs upstream of PI3K can lead to increases in the pathway activity. Mutation activation of EGFR as an activator of PI3K is one of the major alterations in the carcinogenesis of non-small cell lung cancer (NSCLC). EGFR gene amplification and overexpression is observed frequently in glioblastoma, although it is not very common in other cancer types. HER2 is another member of the EGFR family that has been overexpressed and amplified in invasive breast and gastric cancer and, less frequently, in other tumor types, such as lung, colon, biliary, ovarian, and salivary cancer. 
The PIK3CA gene encoding the $p 110 \alpha$ catalytic subunit of PI3K is often mutated in many human cancer types, including breast, endometrial, colorectal, and ovarian tumors. These mutations cluster predominantly in three regions of helical (E542K and E545K) and catalytic (H1047R) domains known as "hot spots" (Figure 4A). The PTEN tumor suppressor gene that antagonizes PI3K signaling is the second-most-frequently mutated gene in human cancer. PTEN loss or mutation have been identified in both spontaneous and heritable cancers, including glioblastoma and endometrial sarcoma. Amplification and activating somatic mutation in the PH domain (E17K) of AKT1 (Figure 4B) have been reported in breast, colorectal, pancreatic, and ovarian cancers [75].

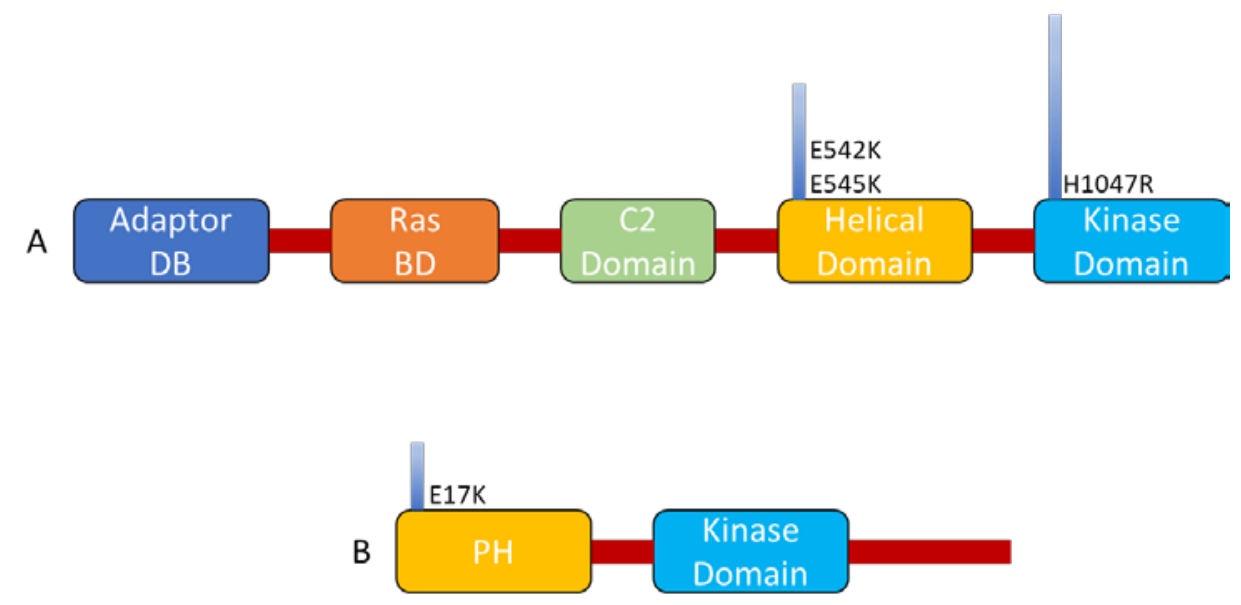

Figure 4. Structural domains of PIK3CA and AKT1 with hotspot mutations. A) PIK3CA protein domain. B) AKT1 protein domain. PH: plekstrin-homology domain. The most common mutations are listed. The heights of the vertical bars indicate the mutation frequency.

The pathway affects different aspects of tumorigenicity including angiogenesis, cell cycle progression, and metastasis. PI3K/AKT/mTOR signaling 
promotes tumor angiogenesis by shifting the homeostatic balance towards proangiogenic factors. Through induction of nitric oxide synthase and inhibition of GSK-3 $\beta$ and FOXO proteins, this signaling pathway increases hypoxia-inducible

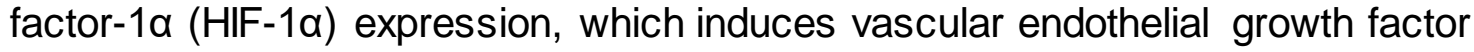
(VEGF) transcriptional activation [76]. In addition, AKT suppresses endogenous angiogenic inhibitor TSP-1 [76]. Thus, the PI3K pathway promotes angiogenesis via overexpression of proangiogenic factors, inhibition of antiangiogenic factors, and induction of factors that promote the stability of developing vasculature.

The PI3K pathway promotes tumor metastasis through several mechanisms. It promotes tumor metastasis by activating matrix metalloproteinases and urokinasetype plasminogen activator, which degrade the extracellular matrix [76]. In addition, the production of chemokines such as C-X-C motif ligand 1 (CXCL-1), cyclooxygenase-2 (COX-2), and interleukin-8 (CXCL-8) in conjunction with promotion of epithelial-mesenchymal transition by NF-KB and repression of Ecadherin allows for the cell motility integral to tumor metastasis [76].

In addition to regulating metastasis, PI3K/AKT/mTOR signaling promotes cell cycle progression by increasing transcription of S-phase entry genes and initiation of translation of messenger RNAs while inhibiting protein regulators of cell cycle progression and pro-apoptotic factors. AKT inhibition of GSK3 $\beta$ and retinoblastoma (RB) protein through cyclin-dependent kinase 4/6 (CDK4/6) results in E2F-mediated transcription of S-phase entry genes [77]. TORC1-mediated activation of S6K1 and inhibition of 4E-BP1, which allows translation of messenger RNAs is required for progression to the S phase [77]. In addition, transition to the S phase occurs by 
inhibiting negative regulators of cell cycle progression such p27 and p21 [77].

Finally, inhibition of pro-apoptotic factors such as FOXO3 proteins, mouse doubleminute 2 homolog, and BAD promotes cell cycle progression [77].

\subsubsection{Targeting PI3K and AKT1 in ER-positive breast cancer}

Molecular characterization of differentially expressed genes in breast cancer patients over the past decades has shown that novel molecular targeted therapies were successful approaches $[78,79]$. In the SOLAR-1 clinical trial, which randomly assigned patients with HR-positive/HER2-negative advanced breast cancer to receive fulvestrant (a selective ER degrader) plus alpelisib (a selective PIK3CA inhibitor) or fulvestrant plus placebo, the median progressionfree survival (PFS) was higher in PIK3CA-positive patients receiving alpelisib compared with placebo (11.0 months vs. 5.7 months, respectively; HR 0.65 95\% $\mathrm{Cl} 0.50-1.25 p=0.00065$ [ [80]. On the basis of this data, the FDA approved alpelisib in combination with fulvestrant for postmenopausal women and men with HR-positive, HER2-negative, PIK3CA-mutated, advanced or metastatic breast cancer after progression on an endocrine-based regimen.

Inhibiting AKT as the central component of frequently disrupted PI3K/AKT signaling has long been an attractive therapeutic approach in cancer. Several compounds for targeting AKT are in clinical development. The two classes of AKT inhibitors include adenosine triphosphate (ATP)-competitive and allosteric inhibitors. The ATP-competitive inhibitors bind to the active site of AKT, blocking ATP binding, whereas allosteric inhibitors bind to the $\mathrm{PH}$ domain, preventing AKT phosphorylation and activation [81]. Although the three AKT isoforms have structural similarity, there 
are differences in their functions, tissue distribution, and substrate specificity [82]; thus, the impact of novel therapeutics on different isoforms in vivo needs further study.

Recently, AKT inhibitors have shown preliminary signal of activity in HRpositive breast cancer with AKT mutations [83]. However, as the PI3K pathway is involved in the survival and proliferation of normal cells, the therapeutic-index of these strategies is quite narrow. For example in the SOLAR-1 trial, adverse events (grade $\geq 3$ ) occurred in $64.4 \%$ of patients receiving alpelisib and fulvestrant compared with $30.3 \%$ of those receiving placebo and fulvestrant [80]. Thus, the toxicity profile of PI3K/AKT pathway-targeted therapies has limited enthusiasm towards their clinical use. Hence, there remains a need for novel therapies targeting vulnerabilities in PIK3CA or AKT1 mutant breast cancer.

\subsection{Cell cycle in breast cancer}

\subsubsection{Cell cycle process}

An uncontrolled cell cycle as a critical regulator of growth and proliferation is one of the hallmarks of cancer [84]. The cell cycle is composed of four ordered phases: G1 (Gap 1) phase, S phase (DNA synthesis), G2 (Gap 2) phase, and M (Mitosis) phase. The progression through these phases is tightly regulated by a group of proteins called cell cycle checkpoints, comprising cyclins and cyclindependent kinases (CDKs), that monitor the integrity of DNA replication and prevent genomic instability which can consequently drive tumorigenesis $[85,86]$. In the presence of DNA damage, checkpoints induce cell cycle arrest through CDK inhibitions to repair the error or trigger cell death or senescence (Figure 5) [87]. 


\subsubsection{The regulation of cell cycle in breast cancer}

In preclinical studies, cyclin D1-dependent CDK4/6 kinases activity is required to form luminal breast cancer and maintain tumorigenesis [88, 89]. Aberrant activation of mitogenic signaling, including PI3K/AKT/mTOR, MAPK/ERK, and ER transcriptional activity (in the case of ER-positive breast cancer), increase CCND1 expression level and/or cyclin D1 protein stability, resulting in the formation of cyclin D1-CDK4/6 complex, which mediates cell cycle progression from G1 phase to S phase (Figure 6) [90-92]. Cyclin D1-dependent CDK4/6 activation leads to the phosphorylation and inactivation of RB which liberates E2F transcription factors and further drives the expression of genes that promote $\mathrm{G} 1$ to $\mathrm{S}$ phase transition (Figures 5 and 6) [93]. 


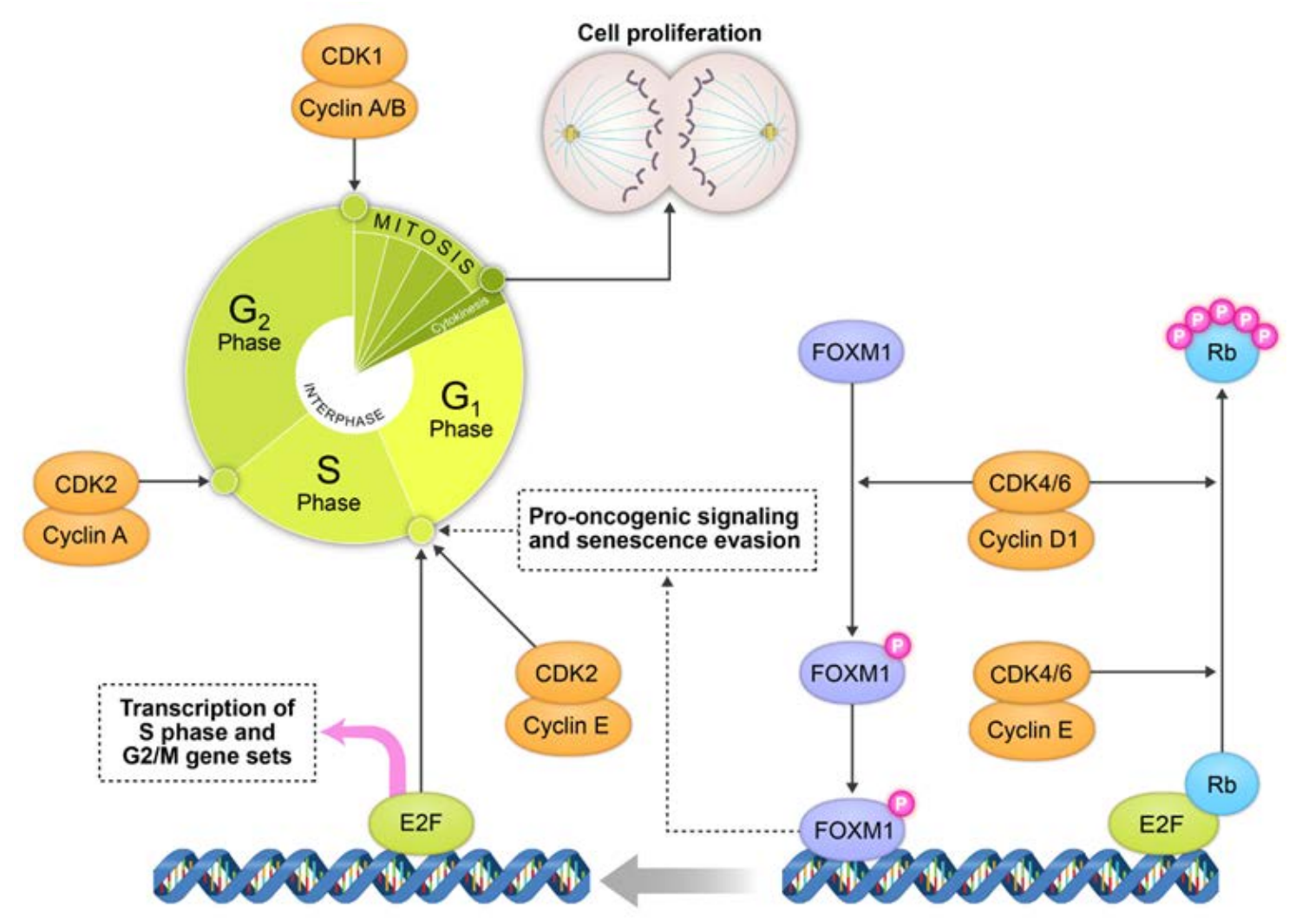

Figure 5. Function of CDK4/6 in cell cycle progression. Active complex of CDK4/6 and cyclin D phosphorylates and inactivates RB protein and then releases transcription factor E2F, triggering the up-regulation of E2F-responsive gene which promotes cell proliferation with cell cycle G1/S transition.

\subsubsection{Role of CDK 4/6 inhibitors in breast cancer}

Given the critical role of CDK4/6 in breast cancer formation, several selective inhibitors with distinct potencies have been developed. The overexpression of cyclin D1 frequently occurs in HR-positive breast cancer, while loss of RB is uncommon. CDK4/6 facilitate $\mathrm{G} 1$ to $S$ phase transition during cell cycle progression, which makes them ideal therapeutic targets in breast tumors with functional RB [94]. Preclinical and clinical antitumor activity in response to CDK4/6 inhibitors has been observed in ER-positive breast cancer [95, 96]. The data support significant 
improvement in PFS and synergistic activity when CDK4/6 inhibitors are combined with endocrine therapy, resulting in the approval of CDK4/6 inhibitors for the treatment of ER-positive, HER2-negative advanced metastatic breast cancer (Figure 6) [97].

\subsection{Approved CDK 4/6 inhibitors for ER-positive, HER2-negative breast cancer treatment}

\subsubsection{Palbociclib}

Palbociclib (PD-0332991) was the first CDK4/6 inhibitor with highly selective enzymatic activity for both CDK4 and CDK6 that showed anti-tumor activity against breast cancer cells [95, 98]. It also exhibited synergy when used with endocrine therapy in ER-positive cell lines. In the PALOMA-1/TRIO-18 phase II clinical trial, the addition of palbociclib to letrozole (an aromatase inhibitor) significantly improved PFS, compared with letrozole alone (20.2 vs 10.2 months; hazard ratio [HR], 0.488; $95 \% \mathrm{Cl}, 0.319-0.748 ; P=0.0004)[95,99]$. This led to the approval of palbociclib plus letrozole as the first-line treatment of postmenopausal women with ER-positive, HER2-negative advanced breast cancer [100]. In the PALOMA-3 randomized phase III clinical trial, 521 postmenopausal women with HR-positive, HER2-negative advanced breast cancer whose cancer had progressed during previous endocrine therapy randomly received palbociclib plus fulvestrant or placebo plus fulvestrant. The median PFS was higher in patients in the palbociclib plus fulvestrant group than the placebo plus fulvestrant group (9.5 vs 4.6 months; $\mathrm{HR}, 0.46 ; 95 \% \mathrm{Cl}, 0.36-$ $0.59 ; P<.0001)[97,101]$. Based on these data, the FDA approved palbociclib in 
combination with fulvestrant for the treatment of HR-positive, HER2-negative advanced or metastatic breast cancer as a second-line treatment $[97,102]$.

\subsubsection{Ribociclib}

Ribociclib (LEE011) is a highly selective CDK4/6 inhibitor with in vitro half maximal inhibitory concentrations (IC50s) of $10 \mathrm{nM}$ and $40 \mathrm{nM}$ for CDK4 and CDK6, respectively [103]. In the MONALEESA-2, a phase III randomized clinical trial, 668 previously untreated postmenopausal women with HR-positive, HER2-negative advanced breast cancer received ribociclib plus letrozole or placebo plus letrozole. Final analysis showed an improved PFS in the ribociclib plus letrozole group compared with the placebo plus letrozole group (25.3 vs 16 months; HR, 0.56; $p<0.001)[104,105]$. On the basis of these data, the FDA approved ribociclib in combination with letrozole for the first-line treatment of postmenopausal women with HR-positive, HER2-negative advanced or metastatic breast cancer [106]. In addition, in the MONALEESA-3 phase III clinical trial, postmenopausal women with HRpositive, HER2-negative advanced breast cancer received ribociclib or placebo plus fulvestrant. The addition of ribociclib improved PFS from 12.8 months to 20.5 months $(H R, 0.593 ; p<0.001)$ which also led to the approval of ribociclib in combination with fulvestrant in this group as first-line or second-line treatment [107].

\subsubsection{Abemaciclib}

Abemaciclib (LY2835219) is also a highly selective CDK4/6 inhibitor that has been shown to inhibit other kinases, including CDK9 and PIM1 $[108,109]$. It induced cell cycle arrest in the G1 phase and inhibited the proliferation of breast cancer cell lines [108]. In the MONARCH-2 phase III clinical trial, 669 patients with HR-positive, 
HER2-negative advanced breast cancer who had previous endocrine therapy were randomly treated with fulvestrant plus either abemaciclib or placebo. Median PFS in the abemaciclib-treated arm improved significantly, compared with the placebotreated arm (16.4 vs 9.3 months; HR, 0.553; 95\% Cl, 0.449-0.681; $P<0.001)$ [110]. In the MONARCH-3 phase III trial, 493 previously untreated patients with HRpositive, HER2-negative advanced breast cancer received abemaciclib plus letrozole or placebo plus letrozole. The abemaciclib group had a longer median PFS (28.18 months) than the placebo group (14.76 months) [111]. Abemaciclib was approved to be used with fulvestrant for the second-line of therapy in women with HR-positive, HER2-negative advanced breast cancer on the basis of the results of the MONARCH-2 trial and was later approved as first-line therapy in combination with an aromatase inhibitor for postmenopausal women on the basis of the MONARCH-3 trial results [112]. 


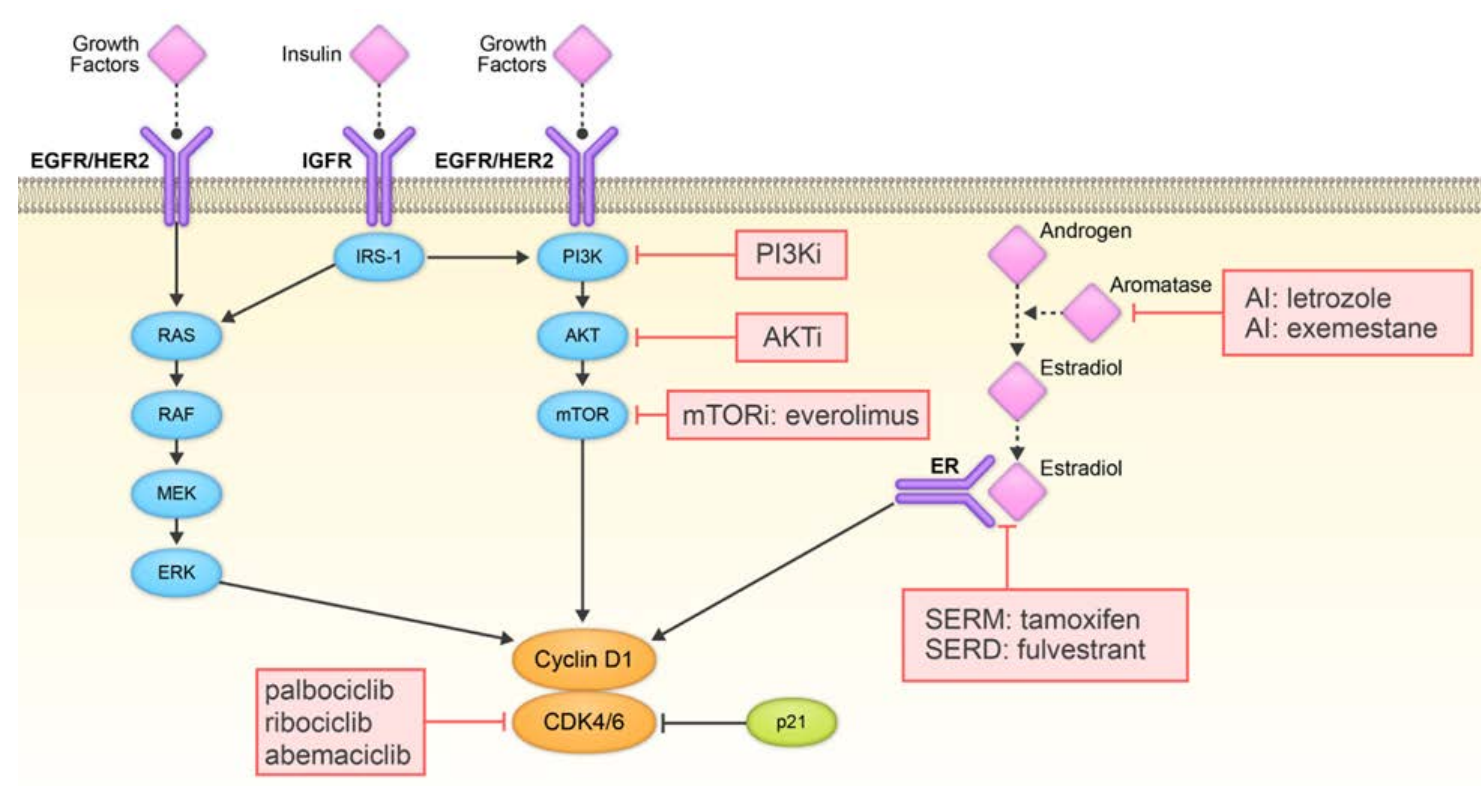

Figure 6. CDK4/6 inhibitors and the upstream mitogenic forces inducing cyclin D1 and activating CDK4/6.Upstream mitogenic signaling pathways, including ER transcriptional activation and, PI3K/AKT/mTOR, and MAP/ERK pathways activation increase CCND1 expression level and/or cyclin D1 protein stability resulting in the formation of cyclin D1-CDK4/6 complex which mediates cell cycle progression from G1 to S phase. Palbociclib, ribociclib and abemaciclib are pharmacologic CDK4/6 inhibitors that directly inhibit the CDK4/6 activity.

\subsubsection{Mechanism of resistance to CDK 4/6 inhibitors in breast cancer}

The development and approval of CDK4/6 inhibitors over the past 2 decades has been a major therapeutic milestone for the treatment of ER-positive, HER2-negative metastatic breast cancer. Multiple studies have shown that ERpositive cell lines with luminal features were most sensitive to palbociclib-the highly selective inhibitor of CDK4/6 — $[95,113]$ but that efficacy was limited by acquired resistance. Mechanisms driving resistance to CDK 4/6 inhibitors have not been clearly defined. Recently, whole-exome sequencing of 59 metastatic 
tumors treated with CDK4/6 inhibitors revealed multiple potential resistance mediators, including RB1 loss, that activate alterations in AKT1, AURKA, CCNE2, RAS, FGFR2, and ERBB2 [114].

Alterations of the PI3K/AKT pathway are crucial to developing resistance to endocrine therapy [115]. Recently, activation of this pathway has been reported to correlate with resistance to CDK4/6 inhibitors [116, 117]. PI3K pathway-dependent activation of non-canonical cyclin D1/CDK2 and the resultant recovery of RB phosphorylation and S- phase entry has been shown to drive resistance to CDK4/6 inhibition [118]. Consequently, the role of PIK3CA and AKT1 inhibitors in overcoming resistance has been investigated in both pre-clinical and clinical studies. Although combining endocrine therapy with CDK4/6 and PIK3CA inhibitors may help prevent acquired $C D K 4 / 6$ resistance in a preclinical setting, this approach may produce considerable toxicity in the clinic [119-121].

\subsection{Goal of the study}

Although recent medical advances in breast cancer treatment have been promising, lack of effective targeted treatments addressing tumor heterogeneity necessitates an improved therapeutic strategy that is based on the genetic profiles of the patients. The PI3K oncogenic pathway is frequently enhanced in ER-positive, HER2-negative breast tumors. This has prompted a sizeable effort toward developing agents that target this pathway at various points. Preclinical studies suggest that the activity of such agents will be enhanced in tumors with PIK3CA or AKT1 mutations $[122,123]$; however, the efficacy of these compounds is not limited to specific genetic alterations. In clinical trials, PI3K pathway inhibitors have limited 
therapeutic efficacy as single agents, and the presence of PIK3CA mutations do not guarantee a dramatic response. Further, neither the Phase III trial of the mTOR inhibitor everolimus plus exemestane (BOLERO 2), nor the Phase II trial of the PI3K inhibitor pictilisib plus fulvestrant (FERGI) in HR-positive breast cancer [124], found differential benefit in patients with PIK3CA mutant tumors [125].

CDK4/6 inhibitors have revolutionized ER-positive, HER2-negative metastatic breast cancer treatment and have become the standard of care in combination with endocrine therapy. However, their efficacies are restricted by intrinsic or acquired resistance. As there is a profound cross-talk between CDK4/6 and the PI3K pathway in the survival and proliferation of normal cells, a therapeutic index making PIK3CA/AKT1 mutant tumors more sensitive to palbociclib than normal tissues may be an efficacious approach. To overcome clinical resistance to CDK4/6 inhibitors, it will be necessary to find additional targets by identifying other potential survival pathways that take advantage of PIK3CA and AKT1 driving mutations to promote ER-positive tumor growth.

The goal of this study was to enhance the antitumor efficacy of palbociclib in ER-positive, HER2-negative breast cancer with PIK3CA or AKT1 mutations with combinational agents that target alternative adaptive survival signaling. This was addressed by using a human kinome shRNA library and nextgeneration sequencing to identify synthetic lethal interactors for palbociclib in the context of ER-positive breast cancer having PIK3CA or AKT1 mutations. Further, the study aimed to investigates the effects of PIK3CA and AKT1 mutations on palbociclib sensitivity. 
CHAPTER 2: MATERIALS AND METHODS 


\subsection{Cell lines}

The isogenic MCF7 ER-positive breast cancer cell line and its derivatives were established through somatic gene targeting technique as previously described [126]. These cell lines were parental MCF7 with one wild-type and 2 E545K alleles (MCF7-PIK3CA), wild type MCF7 with three copies of wild type PIK3CA (MCF7-WT), and wild type MCF7-WT with knock in AKT1-E17K constitutively active mutation (MCF7-AKT1). Authentication of the MCF-7 cell lines was verified using DNA fingerprinting technique based on National Cancer Institute ( $\mathrm{NCl}$ ) public database at MD Anderson Cancer center Core Facility. In addition, droplet digital polymerase chain reaction (PCR) procedure was performed to confirm the status of PIK3CA and AKT1 mutations in the MCF7 isogenic cell lines.

\subsection{Cell culture conditions}

All cells were maintained in Dulbecco's Modified Eagle Medium (DMEM)/F12 media with L-glutamine (Corning cellgro) supplemented with 10\% FBS (fetal bovine Serum, Sigma). The cell culture was carried out at $37^{\circ} \mathrm{C}$ in a humidified incubator with $5 \% \mathrm{CO}_{2}$. The non-transformed immortalized human breast epithelial cell lines, MCF-10A, were maintained on the same standard cell culture condition described. The cells grew in complete MCF-10A growth medium containing mammary epithelial cell basal medium (MEBM) (Lonza Walkersville, Inc.) supplemented with 5\% horse serum (Invitrogen), $20 \mathrm{ng} / \mathrm{ml}$ human epidermal growth factor (hEGF), $10 \mu \mathrm{g} / \mathrm{ml}$ insulin, $0.5 \mu \mathrm{g} / \mathrm{ml}$ hydrocortisone, $100 \mathrm{ng} / \mathrm{ml}$ cholera toxin, and $100 \mu \mathrm{g} / \mathrm{ml}$ streptomycin. Trypsin-ethylenediaminetetraacetic acid (EDTA) (0.25\%) solution (Gibco, Invitrogen) was used for the dissociation and subculturing of the cell lines. 


\subsection{Drug cytotoxicity and proliferation assay}

The pharmacologic drugs palbociclib (LC Laboratories) and 7rh benzamide (Carbosynth) used for cytotoxicity assay. Cells were seeded at a density of $3 \times 10^{3}$ cells per well in 96-well plates to adhere overnight. The next day they were treated with five different concentrations of each drug and dimethyl sulfoxide (DMSO) in a 5fold serial dilution series. Each condition was in triplicate. After 72 hours the cells were fixed with $50 \mu \mathrm{l}$ of $5 \%$ trichloroacetic acid (TCA) per well for 1 hour at $4^{\circ} \mathrm{C}$. After fixing and drying $100 \mu \mathrm{l} 0.4 \%$ sulforhodamine B (SRB) solution was added to each well and the plates were incubated at room temperature for 1 hour. The wells were washed with $1 \%$ acetic acid to remove unbound SRB dye. $100 \mu$ of $10 \mathrm{mM}$ Tris base solution ( $\mathrm{pH} 10)$ was added to each well to solubilize the protein-bound dye.

The optical density (OD) value was measured at $510 \mathrm{~nm}$ in Synergy 4 microplate reader (Bio Tek). The data were analyzed to determine the half maximal inhibitory concentration (IC50) and combination index (CI) based on the Median Effect methods using the dose-effect analyzer software CalcuSyn 2.0. To measure proliferation rate, cells were seeded in triplicate at a density of $2 \times 10^{3}$ cells per well in several 12-well plates. Cells were harvested every two days and diluted 1:1 with Trypan Blue solution to count the viable cells using Countess II automated cell counter (Life Technologies). Experiment was repeated three times.

\subsection{Clonogenic survival assay}

Cells were seeded at $1 \times 10^{3}$ cells per $60 \mathrm{~mm}$ plate in triplicate and treated with different doses of palbociclib and 7rh for 3 weeks until the control approached confluency. Fresh medium containing the drug was added to the wells twice a week. 
Plates containing the colonies were then washed twice with phosphate-buffered saline (PBS) and fixed in 10\% formalin for 15 minutes at room temperature. After fixation process, the cells were stained in $0.05 \%$ crystal violet with $25 \%$ methanol for 10 minutes. After the removal of crystal violet, the plates were washed several times with water before image acquisition using a scanner. NIH ImageJ v.1.46 software was used to count the colonies.

\subsection{FACS analysis of cell cycle}

Cells were seeded at $5 \times 10^{5}$ cells per $100 \mathrm{~mm}$ plate in triplicate and treated with various doses of palbociclib and 7rh. After 48 hours, the cells were collected and fixed in cold $70 \%$ ethanol, then washed twice with PBS. To remove doublestranded RNA, $1 \mathrm{U}$ of RNase, DNase free was added to the cell suspensions and incubated at $37^{\circ} \mathrm{C}$ for 1 hour. The cells were stained with propidium iodide (Roche), stored at $+2^{\circ} \mathrm{C}$ to $+8^{\circ} \mathrm{C}$, and protected from light until flow cytometric analysis. Percentages of cells in different phases of the cell cycle were determined using a Gallios flow cytometer (Beckman Coulter Inc.) and analyzed with Kaluza analysis software (Beckman Coulter Inc.).

\subsection{Soft agar assay}

MCF-10A cells were seeded at $1 \times 10^{5}$ cells per well of six-well plates. The wells were coated with $0.5 \%$ agarose/1X medium composed of low-melting agar (Difco Agar Noble, BD), DMEM powder (Sigma), and FBS (base agar) and overlaid with $2 \mathrm{ml} 0.35 \%$ agarose/1X medium solution containing the cells (top agar). The soft-agar cultures were maintained at $37^{\circ} \mathrm{C}$ in a humidified incubator with $5 \% \mathrm{CO}_{2}$, and the top agar medium was replaced with fresh medium every other day. Colonies 
were then fixed with $0.05 \%$ iodonitrotetrazolium chloride (INT) prepared at $0.5 \mathrm{mg} / \mathrm{ml}$ in PBS and incubated overnight at $37^{\circ} \mathrm{C}$. Images for both bright field and red fluorescent protein (RFP) were obtained by using FluorChem 8900 (Alpha Innotech).

\subsection{Immunoblotting}

The primary antibodies used are listed in Table 2 . Whole cell protein extracts prepared with Laemmli buffer, were heated in sample buffer for 5 min at $95^{\circ} \mathrm{C}$. Approximately $30 \mu \mathrm{g}$ of each sample loaded in 10\% SDS-PAGE and transferred to nitrocellulose membranes (Bio-Rad). After blocking in bovine serum albumin (BSA) for 1 hour, the membranes were probed with the indicated antibodies diluted in Trisbuffered saline with $0.1 \%$ Tween 20 (TBST) with 5\% BSA (Sigma) and incubated overnight at $4^{\circ} \mathrm{C}$. The membranes were then washed $3 \mathrm{X}$ in TBST and incubated with secondary antibody for 1 hour at room temperature. Detection was performed by visualizing the membranes using LI-COR Infrared Imaging System (LI-COR Biotechnology). 


\begin{tabular}{|l|l|c|}
\hline \multicolumn{1}{|c|}{ Protein } & \multicolumn{1}{c|}{ Vendor } & Catalog \# \\
\hline AKT & Cell Signaling Technology & 2920 \\
\hline pAKT-T308 & Cell Signaling Technology & 13038 \\
\hline pAKT-S473 & Cell Signaling Technology & 4060 \\
\hline 4EBP1 & Cell Signaling Technology & 9452 \\
\hline p-4EBP1-T37/46 & Cell Signaling Technology & 9459 \\
\hline p-4EBP1-T70 & Cell Signaling Technology & 9455 \\
\hline p-4EBP1-S65 & Cell Signaling Technology & 9456 \\
\hline ERK 1/2 & Cell Signaling Technology & 9102 \\
\hline pERK 1/2-T202-Y204 & Cell Signaling Technology & 4370 \\
\hline pGSK3b-S9 & Cell Signaling Technology & 5558 \\
\hline GSK3b & Cell Signaling Technology & 12456 \\
\hline PRAS40 & Cell Signaling Technology & 2691 \\
\hline p-PRAS40-T246 & Cell Signaling Technology & 13175 \\
\hline 70-S6K & Cell Signaling Technology & 9202 \\
\hline p70-S6K-T389 & Cell Signaling Technology & 9234 \\
\hline S6 & Cell Signaling Technology & 2217 \\
\hline pS6-S235/236 & Cell Signaling Technology & 4858 \\
\hline DDR1 & Cell Signaling Technology & 5583 \\
\hline FoxM1 & Cell Signaling Technology & 5436 \\
\hline PLK-1 & Cell Signaling Technology & 4513 \\
\hline P21 & Cell Signaling Technology & 2947 \\
\hline p-RB-S807/811 & Cell Signaling Technology & 9307 \\
\hline Cyclin D1 & Cell Signaling Technology & 2978 \\
\hline Cyclin E2 & Cell Signaling Technology & 4132 \\
\hline RRM2 & Invitrogen & PA5-27856 \\
\hline c-Myc & Cell Signaling Technology & 5605 \\
\hline LC3B I/II & Cell Signaling Technology & 2775 \\
\hline beta-Actin & Sigma Aldrich & A2229 \\
\hline & & \\
\hline
\end{tabular}

Table 2. List of primary antibodies.

\section{8 shRNA library screening}

The human kinome (hkinom) shRNA library in pRSI vector tagged with GFP cDNA and expressing puromycin resistance gene, was provided by the Center for Co-Clinical Trials (CCCT) at the University of Texas MD Anderson Cancer Center 
(Human DECIPHER, Cellecta Inc.). Prior to transduction the optimal concentrations of polybrene and puromycin were calculated based on toxicity kill curve experiment. Polybrene toxicity titration in target cells was performed by growing cells in culture medium with a range of polybrene concentrations $(0 \mu \mathrm{g} / \mathrm{ml}, 1 \mu \mathrm{g} / \mathrm{ml}, 2 \mu \mathrm{g} / \mathrm{ml}, 4$ $\mu \mathrm{g} / \mathrm{ml}, 6 \mu \mathrm{g} / \mathrm{ml}, 8 \mu \mathrm{g} / \mathrm{ml}$ ) for 24 hours then replaced with polybrene-free culture medium. Cells grew for an additional 72 hours and toxicity was checked by counting viable cells. The highest concentration of polybrene that results in less than $10 \%$ cell toxicity compared to no polybrene was selected for shRNA library transduction. Puromycin kill cure was generated by treating the target cells with a range of puromycin concentrations $(0 \mu \mathrm{g} / \mathrm{ml}, 0.5 \mu \mathrm{g} / \mathrm{ml}, 1 \mu \mathrm{g} / \mathrm{ml}, 2 \mu \mathrm{g} / \mathrm{ml}, 5 \mu \mathrm{g} / \mathrm{ml}, 10 \mu \mathrm{g} / \mathrm{ml})$. The cells grew under standard conditions and the lowest concentration that kills $>95 \%$ of cells in 48 hours was selected for screening.

Before the lentiviral library transduction, a range of $\mathrm{MOI}(0,0.15,0.3,0.5,1$, and 2) was used to determine the desired one for $20 \%$ transduction. The number of viral particles required to achieve different $\mathrm{MOI}$ was calculated as below using the stock virus titer of $2.05 \times 10^{8} \mathrm{TU} / \mathrm{ml}$ :

a. Number of cells seeded $\mathrm{x}$ desired $\mathrm{MOI}=\#$ of TU required

b. \# of TU / Functional titer of pRSI virus = amount of virus to add to each well.

c. $\mathrm{TU} / \mathrm{ml}=(\#$ of cells at Transduction $) \times \mathrm{MOI} /(\mathrm{ml}$ of Viral Stock used at Transduction)

For reverse transduction, $1 \times 10^{5}$ cells for approximately $95 \%$ confluency in 72 hours added in each well of 6-well plate in media containing $8 \mu \mathrm{g} / \mathrm{ml}$ polybrene and 
transduced by adding the calculated amount of viral particle to each plate. The next day viral media was replaced with normal growth media (no polybrene). After an additional 48 hours, the $\%$ of infected cells was determined by observing the $\%$ of GFP positive cells in each transduced cell sample using flow cytometry. The MOI and viral integration was calculated based on Poisson distribution and the MOI required (in the range of $0.2-1.5$ ) for $20 \%$ transduction efficiency was determined for the eventual complexity test drop-out screen.

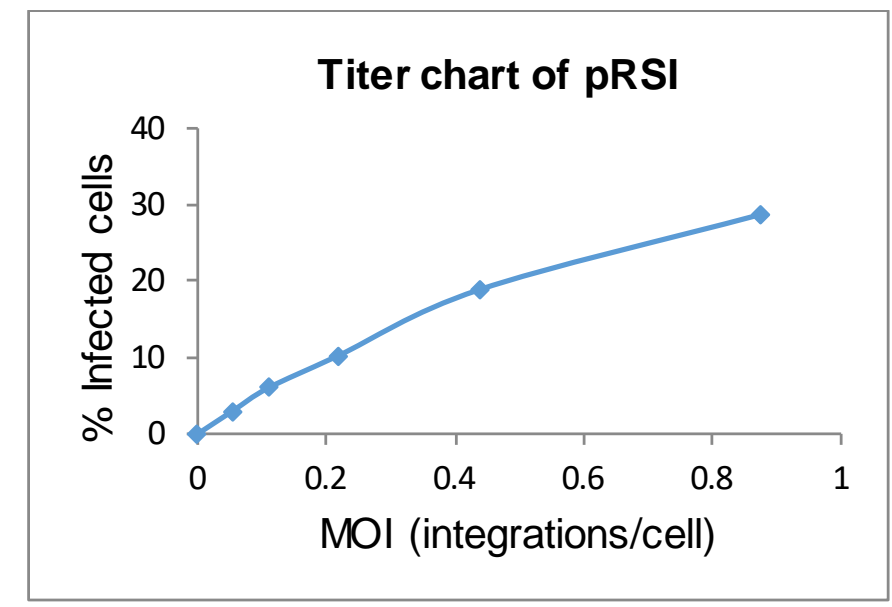

Figure 7. MOI and viral integration calculated based on Poisson distribution.Cells were transduce in suspension by adding the calculated number of viral particles (described above) equivalent to a range of $\mathrm{MOI}(0$ to 1 ). 48-72 hours post transduction, the $\%$ of infected cells was determined by flow cytometry by observing the $\%$ of GFP+ cells.

Using the calculated MOI (Figure 7), viral transduction was performed as before and the transduced cells cultured in media containing $2 \mu \mathrm{g} / \mathrm{ml}$ puromycin for two weeks in the presence of drug or vehicle. A reference cell population was frozen 
(stored at $-80^{\circ} \mathrm{C}$ ) at the beginning of the two weeks treatment period to eventually compare the shRNA barcode abundance to the drug treated cells.

\section{9 shRNA gene silencing and plasmid overexpression}

For silencing, pLKO-puro lentiviral vectors containing DDR1 and control shRNA constructs were obtained from shRNA Mission (Sigma-Aldrich). To generate viral particles, 293T cells were transfected with shDDR1 and non-targeting control shRNA along with psPAX2 (packaging) and PCMV-VSV-G (envelope) expression constructs using Lipofectamine 2000 transfection reagent (Invitrogen).

The viral supernatants were harvested at 24 and 48 hours post transfection, centrifuged at $500 \mathrm{~g}$ for 5 minutes to pellet any packaging cells and filtered through $0.45 \mu \mathrm{m}$ PES filter. Prior to transduction the optimal dose of antibiotic selective marker was obtained by treating target cells with a range of puromycin concentrations ( $0 \mu \mathrm{g} / \mathrm{ml}, 0.5 \mu \mathrm{g} / \mathrm{ml}, 1 \mu \mathrm{g} / \mathrm{ml}, 2 \mu \mathrm{g} / \mathrm{ml}, 5 \mu \mathrm{g} / \mathrm{ml}, 10 \mu \mathrm{g} / \mathrm{ml})$ for 48 hours and toxicity was checked by counting viable cells to determine the lowest dose that kill more than $95 \%$ of the cells. The day before transduction, $1 \times 10^{5}$ cells seeded in each well of 6-well plates to exhibit 30-40 \% confluency at the time of transduction. The lentiviral particles supplemented with $8 \mu \mathrm{g} / \mathrm{ml}$ polybrene added to the cells and incubated overnight. One days after infection cells treated with medium containing 2 $\mu \mathrm{g} / \mathrm{ml}$ puromycin for 2 more weeks.

For selection of stable cells, puromycin added to the cell culture medium and replaced every 3 days until resistant colonies identified. The antibiotic resistant pools were examined for DDR1 protein expression by western blot and frozen at each cell passage. DDR1 wild type expression vector, pHAGE-puro-DDR1, was generated by 
Gateway LR reaction from pDNOR-DDR1 to pHAGE-puro vector. DDR1 mutants expression vectors were generated by PCR-based mutagenesis as described before [127], and cloned into pHAGE-puro vector by Gateway LR reaction.

\subsection{Quantitative real-time PCR}

Total cellular RNA was extracted (Qiagen RNeasy Mini Kit) and $1 \mu \mathrm{g}$ was used for cDNA synthesis using SuperScript III First-Strand Synthesis kit (Invitrogen) according to manufacturer's protocol. Quantitative real-time (qRT)-PCR reaction was performed in SYBR GreenER qPCR SuperMix cocktail (Invitrogen) using the primers for the gene of interest [128] (obtained from PrimerBank at The center for Computational and Integrative Biology, Harvard Medical School). The mRNA levels were measured with 7500 Real-Time PCR System (Applied Biosystems). Each qRTPCR experiment was repeated three times in technical triplicate and mRNA levels were normalized to GAPDH expression applying $\Delta \Delta \mathrm{Ct}$ relative quantification method. The following primers were used for qRT-PCR:

PrimerBank ID: 378404907c2

GAPDH-Forward: 5' ACAACTTTGGTATCGTGGAAGG 3'

GAPDH-Reverse: 5' GCCATCACGCCACAGTTTC 3'

PrimerBank ID: 321400062c2

DDR1-Forward: 5' CCGACTGGTTCGCTTCTACC 3'

DDR1-Reverse: 5' CGGTGTAAGACAGGAGTCCATC 3'

PrimerBank ID: $340545541 c 3$

FoxM1-Forward: 5' ATACGTGGATTGAGGACCACT 3'

FoxM1-Reverse: 5' TCCAATGTCAAGTAGCGGTTG 3' 
PrimerBank ID: 34147632c3

PLK1-Forward: 5' CCTGCACCGAAACCGAGTTAT 3'

PLK1-Reverse: 5' CCGTCATATTCGACTTTGGTTGC 3'

PrimerBank ID: 310832423c1

CDKN1A (P21)-Forward: 5' TGTCCGTCAGAACCCATGC 3'

CDKN1A (P21)-Reverse: 5' AAAGTCGAAGTTCCATCGCTC 3'

Real-time instrument was programmed as shown below:

- $50^{\circ} \mathrm{C}$ for 2 minutes

- $95^{\circ} \mathrm{C}$ for 10 minutes

40 cycles of:

- $95^{\circ} \mathrm{C}$ for 15 seconds

- $60^{\circ} \mathrm{C}$ for 60 seconds

\subsection{Reverse phase protein array (RPPA) analysis}

The RPPA was performed in the MD Anderson Cancer Center RPPA core facility. To prepare samples for RPPA, the xenograft tumor tissues were dissected on dry ice and lysed in lysis buffer containing 1\% Triton X-100, 50mM HEPES, pH 7.4, $150 \mathrm{mM} \mathrm{NaCl}, 1.5 \mathrm{mM} \mathrm{MgCl} 2,1 \mathrm{mM}$ EGTA, 100mM NaF, 10mM Na pyrophosphate, 1mM Na3VO4, 10\% glycerol, protease and phosphatase inhibitors (Roche). The protein concentration was determined by BCA assay (Pierce) and adjusted to $1.5 \mu \mathrm{g} / \mu \mathrm{l}$ for all the samples using lysis buffer for dilution. The cell lysate were mixed with 4 X SDS sample buffer containing 40\% Glycerol, 8\% SDS, $0.25 \mathrm{M}$ 
Tris-HCL pH 6.8, and $0.1 \%$ beta-mercaptoethanol (B-Me) at the ratio of 1 to 4 then boiled for 5 minutes at $100^{\circ} \mathrm{C}$ and stored at $-80^{\circ} \mathrm{C}$.

The lysates were printed on nitrocellulose-coated slides using Aushon Biosystems 2470 arrayer and probed with a total of 400 primary antibodies followed by the appropriate biotinylated secondary antibodies for detection. The slides were scanned with Array-Pro Analyzer software for generation of spot intensity. SuperCurve GUI [129] was used to estimate relative protein level in log2 scale. RPPA slides quality was determined with a QC metric [130] and only the slides with 0.8 on a $0-1$ scale were included for further processing. Protein measurements were corrected using median-centering across antibodies and samples with low protein level were excluded for further analysis. The data were normalized and centered to present the protein mean expression value in Log2.

\subsection{In vivo experiments}

All animal experiments were approved by the University of Texas MD Anderson Cancer Center Institutional Review Board and in accordance with the Institutional Animal Care and Use Committee (IACUC) guidelines. MCF7-PIK3CA cells $\left(10 \times 10^{6}\right)$ were re-suspended in $200 \mu$ of 1:1 matrigel (Corning Life Sciences) in DMEM medium without serum and injected into mammary fat pads of 6 weeks old female NSG (NOD scid gamma) mice (The Jackson Laboratory) supplemented with $17 \beta$-estradiol pellets. The treatment started when tumor mean volumes reached approximately $200 \mathrm{~mm}^{3}$ and the animals were randomly assigned to four treatment groups (5 mice in each group). Drugs were dissolved in 1\% carboxymethyl cellulose,

$0.25 \%$ Tween 80 . Animals were treated daily with oral administration of palbociclib 
(25 mg/kg) once a day and 7rh ( $25 \mathrm{mg} / \mathrm{kg}$ ) twice a day. The tumor sizes were measured with a caliper every 4 days and the volumes were calculated using $\mathrm{V}$ $\left(m^{3}\right)=a b^{2} / 2$ equation, where "a" is the largest diameter and "b" is the smallest diameter. At the end of the experiment, mice were euthanized 4 hours after the last treatment and the tumors were extracted for further analysis in IHC and RPPA. Compressed $\mathrm{CO}_{2}$ gas in cylinders was used for the euthanasia chamber at the flow rate of $20 \%$ of the chamber volume per minute.

\subsection{Immunohistochemical staining of human xenografts}

The harvested xenograft tumor tissues were fixed in 10\% Neutral Buffered Formalin (NBF) for 24 hours at room temperature and embedded in paraffin (Sigma). The FFPE tissues were cut into $4 \mu \mathrm{m}$, mounted on Plus glass slides and evaluated for hematoxylin and eosin (H\&E) staining.

The paraffin-embedded sections were deparapffinized in xylene and hydrated in graded alcohols $(100 \%, 95 \% \mathrm{ETOH})$. The endogenous peroxidase activity was blocked with $3 \% \mathrm{H}_{2} \mathrm{O}_{2}$ in water. Antigen retrieval was performed in $10 \mathrm{mM}$ Citrate Buffer ( $\mathrm{pH}$ 6.0) in microwave for 15 minutes under standardized conditions then cooled down for 20 minutes and washed with water. Non-specific antibody binding was blocked by incubating slides with Biocare Blocking Reagent \# BS966M (Casein in buffer) for 10 minutes. Immunostaining was done by incubating the slides for 15 minutes at room temperature (Ki67) or overnight at $4^{\circ} \mathrm{C}$ (phospho-Rb and P21) using the primary antibodies against Ki67 (clone MIB-1, mouse monoclonal from DAKO cat. \# M7240, at 1:100 dilution), phospho-Rb (Ser-780) (EPR182N, rabbit monoclonal from Abcam cat. \# ab173289, at 1:100 dilution), P21 (F-5, mouse 
monoclonal from Santa Cruz \# sc-6246, at 1:50 dilution) with negative and positive controls for each experiment.

The slides were incubated with Envision plus labeled polymer, anti-rabbitHRP (Dako) for 30 minutes at room temperature and visualized using Dako DAB monitoring staining development kit (Sigma). The tissue processing and immunohistochemical staining were carried out at the MD Anderson Cancer Center Research Histology Core Laboratory (RHCL) and Research Histology, Pathology and Imaging Core (RHPI).

\subsection{Immunohistochemical scoring and imaging}

The immunohistochemical staining was evaluated by a pathologist under Olympus BX46 microscope. For Ki-67 and phospho-Rb the percentage of positive cells in the tumor was estimated. For $\mathrm{P} 21$ and $\mathrm{Bcl}-2$ the percentage of positivity (0\% to $100 \%)$ and the staining intensity $(0=$ no staining, $1+=$ weak staining, $2+=$ moderate staining, and $3+=$ strong staining) were evaluated resulting in an $\mathrm{H}$-score by multiplying percentage and intensity (range: 0-300). Images were obtained by scanning the slides at 40X using Olympus DP27 Microscope Digital Camera.

\subsection{Statistical analysis}

Comparison between two groups was done using two-way student $t$ test with GraphPad Prism software. All the in vitro experiments were performed three times and the data were presented as mean \pm SE (SEM). The $p$ value that was used as a cutoff for determining significance was 0.05 ( $p$ value $<0.05)$. For RPPA data analysis, differentially expressed proteins (DEPs) between treatment groups were 
identified by fitting linear model and making contrast with $\mathrm{R}$ package Limma. False discovery rate (FDR) of 0.05 was used as the cutoff value. For in vivo data, two-way ANOVA and Tukey's multiple comparison test were applied to analyze the significance of tumor volume in different treatment groups. P-value calculated based on ANOVA: $0.1234(\mathrm{~ns}), 0.0332\left({ }^{*}\right), 0.0021\left(^{(\star}\right), 0.0002\left(^{(\star \star}\right)$ and $<0.0001\left({ }^{(\star \star *}\right)$. 


\section{CHAPTER 3: PIK3CA AND AKT1 MUTATIONS ENHANCE BREAST CANCER CELL GROWTH AND REDUCE PALBOCICLIB SENSITIVITY IN VITRO}

\subsection{INTRODUCTION}

Molecular alterations in the PI3K/AKT pathway occur in more than $30 \%$ of ERpositive invasive breast tumors [131]. These genetic mutations include genes encoding the PI3K catalytic subunits $p 110 \alpha(P I K 3 C A)$ and the PI3K effector AKT1. Aberrant PI3K/AKT signaling is implicated in the pathogenesis of ER-positive breast cancers [132] and therefore is a major focus of drug development.

Despite the efficacy of the available small-molecule inhibitors for targeting different components of PI3K pathway, the contribution of PIK3CA and AKT1 genetic alterations to targeted therapy is not well understood. Even though PI3K pathway mutations occur at a high frequency in breast cancer, little work has been done to elucidate the biological and clinical significance of PIK3CA and AKT1 alterations. To address functional consequences of PIK3CA and AKT1, we examined the differential effects of these "hot spot" mutations on proliferation and tumor growth.

CDK4/6 plays a key role in the proliferation of breast cancer as well as the development of normal breast tissue, which establishes a strong rationale for targeting these kinases. CDK4/6 inhibitors have profoundly impacted ER-positive, HER2-negative metastatic breast cancer treatment and have become the standard 
of care in combination with endocrine therapy. However, their efficacy is restricted by intrinsic or acquired resistance. Both preclinical and clinical data support the activation of upstream PI3K mitogenic pathway as a potential mechanism of resistance $[118,133]$. Thus, we determined the effect of PIK3CA and AKT1 mutations on palbociclib sensitivity, which will ultimately help to identify predictive biomarkers and optimal targeted therapies in combination with CDK4/6 inhibitors.

The use of isogenic cell lines is a complementary approach to study the influence of genotype on drug sensitivity. This will eliminate the effect of other alterations that could impact treatment efficacy and provide a model system with a uniform phenotypic effects. To date, none of the identified human breast cancer cell lines has an $A K T 1$ mutation and thus the use of $A K T 1$ knock-in model helps us to better assess the effect of this mutation.

\subsection{RESULTS}

\subsubsection{Validation and authentication of MCF7 isogenic cell lines}

The isogenic MCF7 ER-positive breast cancer cell line and its derivatives were established by using a somatic gene-targeting technique, as previously described [126]. These cell lines were parental MCF7 with one wild-type and two E545K alleles (MCF7-PIK3CA), wild-type MCF7 with three copies of wild-type PIK3CA (MCF7-WT), and wild-type MCF7-WT with knock-in AKT1-E17K constitutively active mutation (MCF7-AKT1). Authentication of the MCF-7 cell lines was verified by using a DNA fingerprinting technique based on a National Cancer Institute (NCI) public database (Table 3). In addition, droplet digital PCR procedure was performed to confirm the status of PIK3CA and AKT1 mutations in the MCF7 
isogenic cell lines. The droplet digital PCR revealed that all mutants were expressed at the expected ratios to wild-type transcripts (Figures $8 \mathrm{~A}$ and $\mathrm{B}$ ).

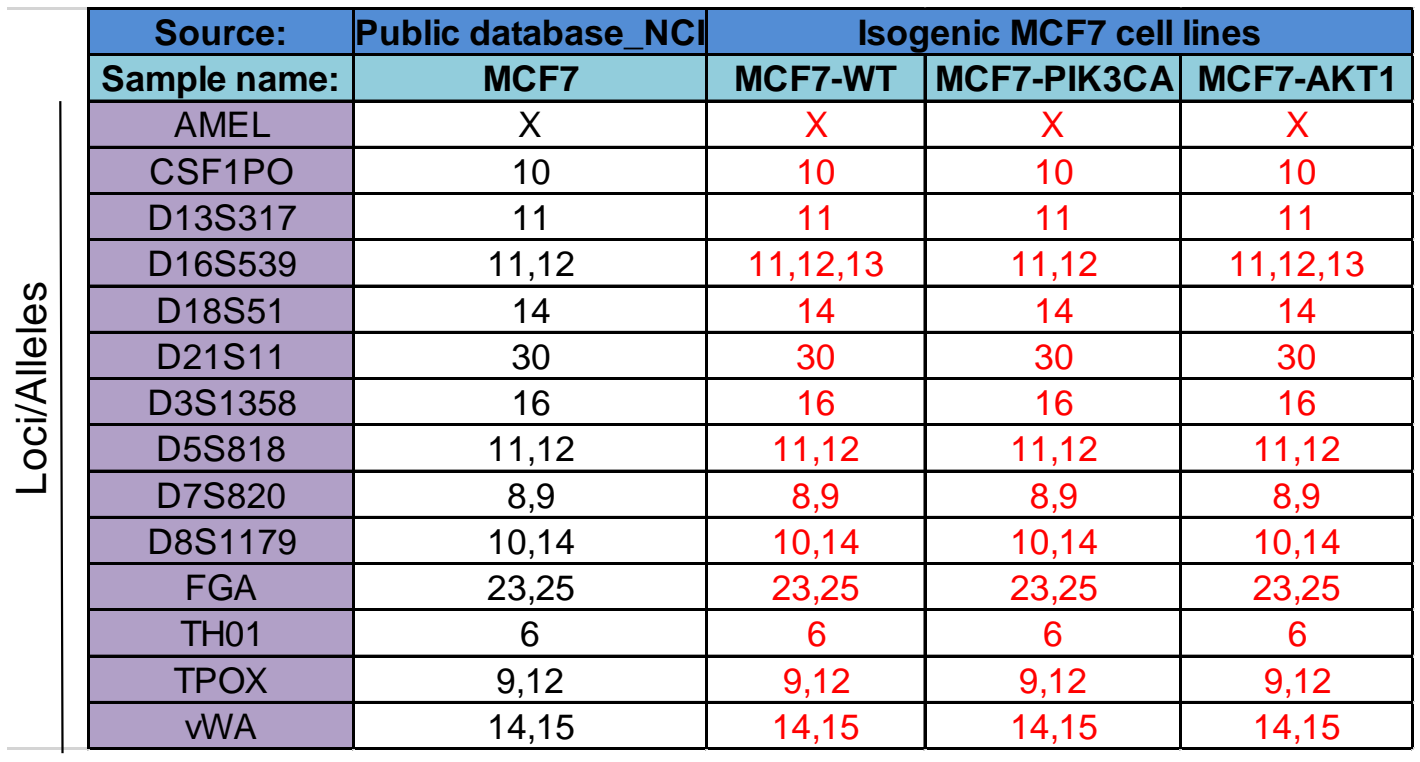

Table 3. DNA fingerprinting of the isogenic MCF7 cell lines. DNA fingerprinting using the CCSG databases verified the authentication of the MCF7 cell lines. CCSG database is a comprehensive databases of short tandem repeat (STR) profiles. The database includes profiles from 20 public database/ publications and the cell lines developed by MD Anderson research labs. The database contains over 2500 unique human cancer cell line STR profiles, one of the largest cancer cell line STR online search database in the world. 


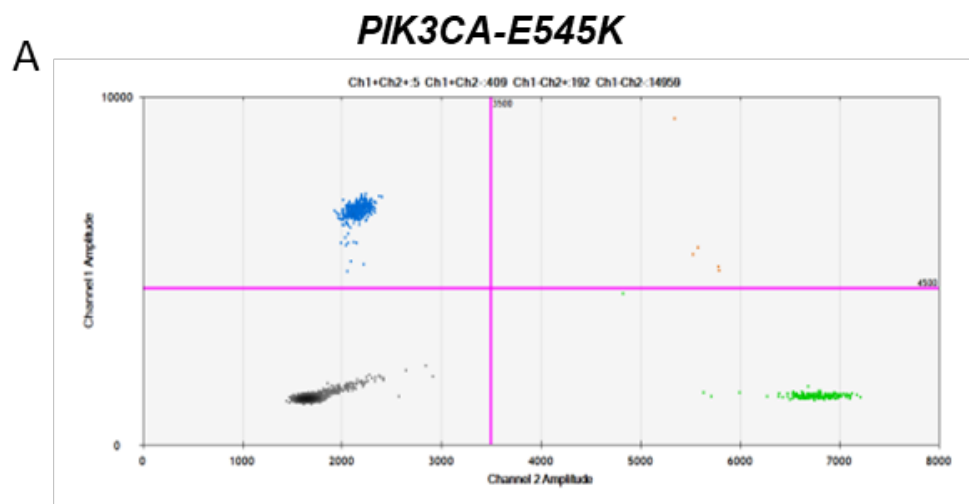

PIK3CA-E545K/PIK3CA wild type ratio $=2.1$

WT

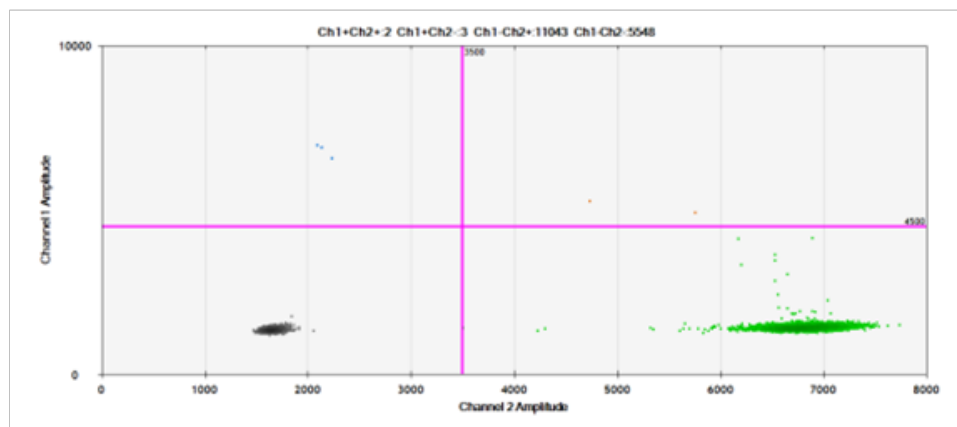

PIK3CA-E545K/PIK3CA wild type ratio > 0.0001

AKT1-E17K

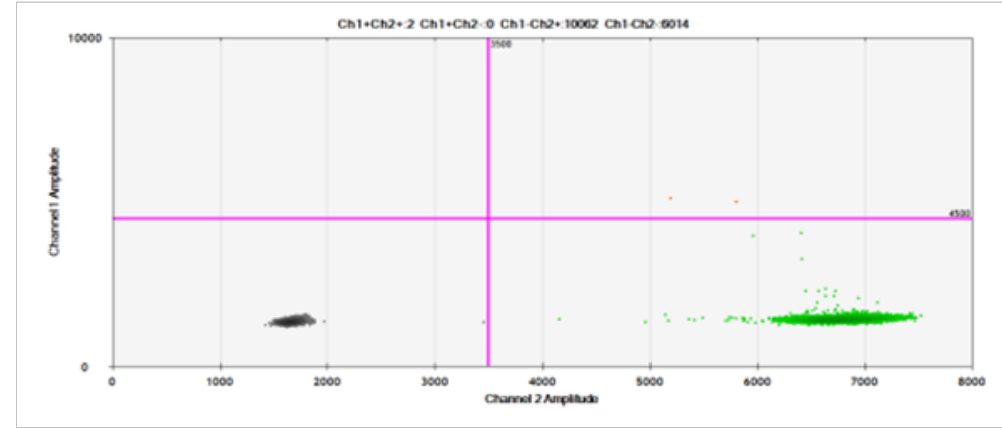

PIK3CA E545K/PIK3CA wild type ratio > 0.0001

44 


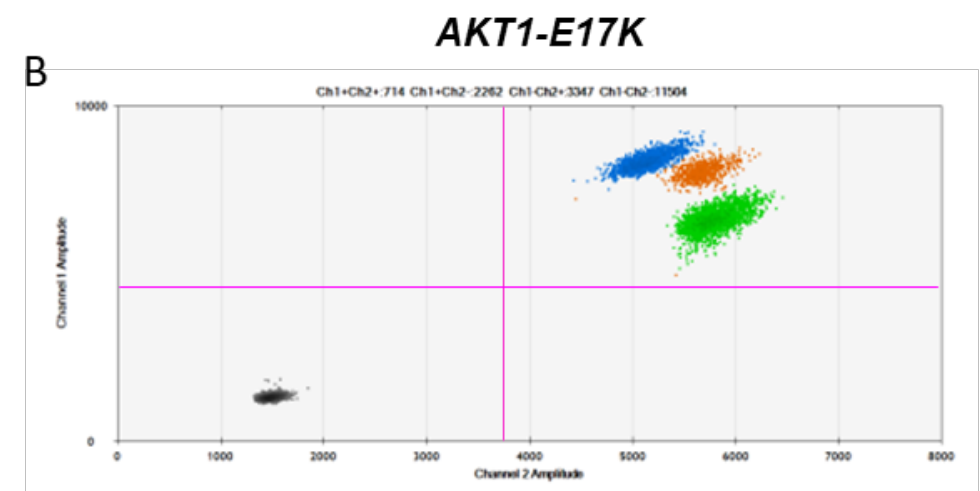

AKT1-E17K/AKT1 wild type ratio $=0.807$

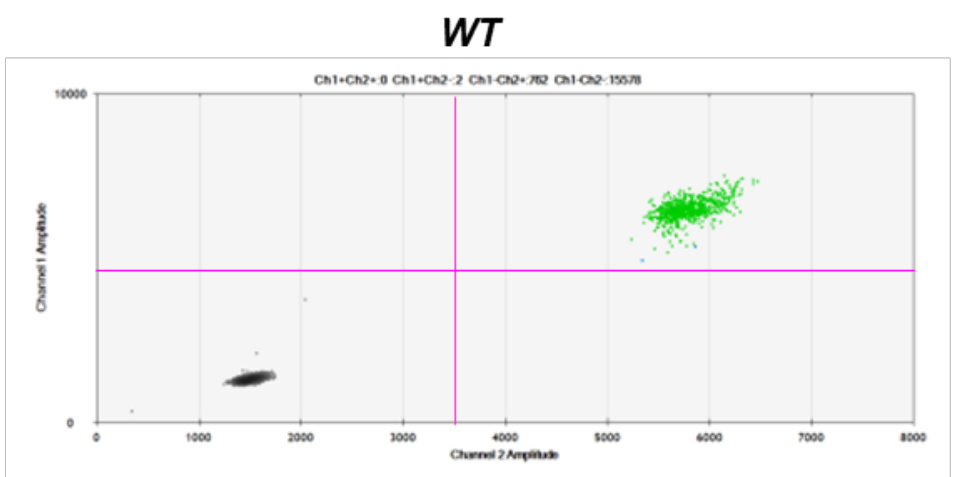

AKT1-E17K/AKT1 wild type ratio > 0.001

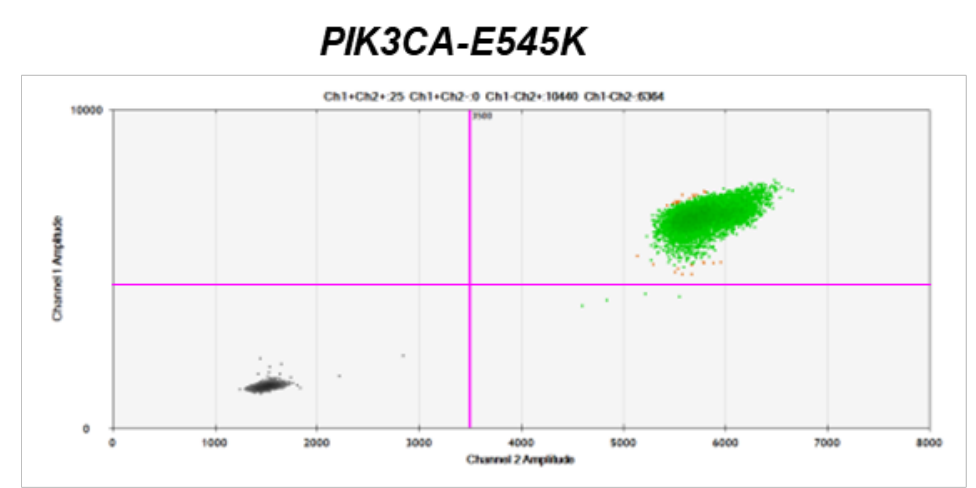

AKT1-E17K/AKT1 wild type ratio > 0.001

Figure 8. Validation of PIK3CA and $A K T 1$ alterations in MCF7 isogenic cell lines.Droplet digital PCR was performed using the genomic DNA isolated from the MCF7 isogenic cell lines to detect the presence of A) PIK3CA-E545K and B) AKT1E17K mutations. 


\subsubsection{Growth characterizations and effects of PIK3CA and AKT1 mutations in vitro and in vivo}

First, we investigated the effects of PIK3CA and AKT1 mutations on cell growth, by assessing growth assay in full serum and low serum using the previously described isogenic MCF7 cell line. The presence of mutant PIK3CA in MCF7PIK3CA provided growth advantage in these cell lines in both serum conditions (Figures 9A-C). The introduction of AKT1-E17K mutation on MCF7-WT with wildtype PIK3CA resulted in an increased proliferation rate. MCF7-AKT1 grew at an intermediate rate between mutant and wild-type PIK3CA. We also tested the in vivo growth ability of these cell lines in nude mice implanted with estradiol pellets.

PIK3CA and AKT1 mutant cells readily formed tumors at similar rates, whereas MCF7-WT tumor growth was slower (Figure 9D). In addition, PIK3CA and AKT1 mutant cells stimulated greater colony formation than did wild-type PIK3CA cells (Figure 10A). PIK3CA and AKT1 induced activation of AKT and showed increased phosphorylation of serine-473 and threonine-308, as detected by immunoblot. In turn, AKT activation enhanced the phosphorylation of known AKT target proteins, including GSK3 $\beta$ and PRAS40 (Figure 10B). 
A

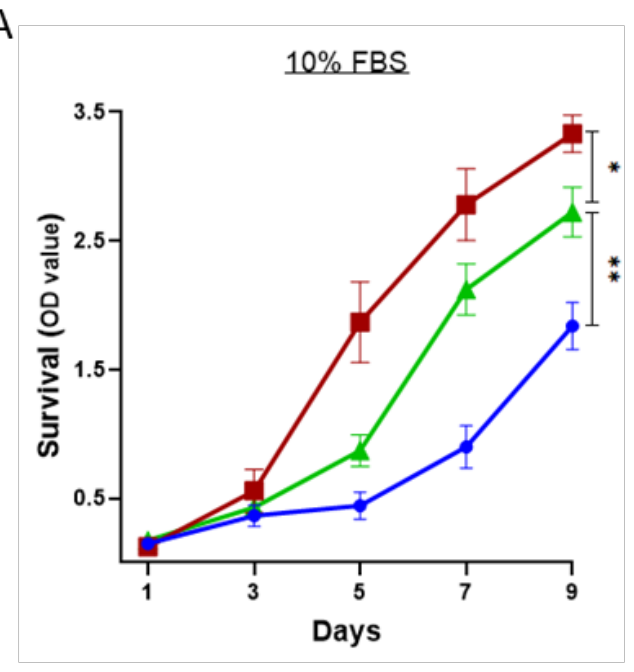

C

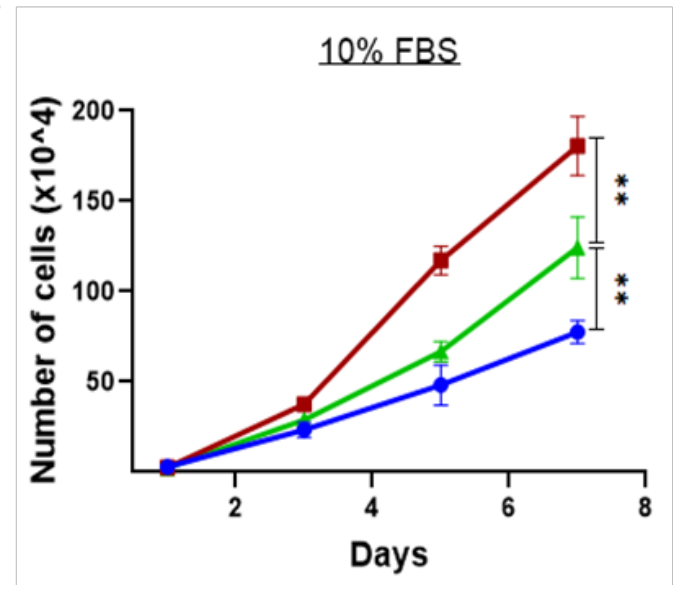

B $5 \%$ CSS $+5 n M E 2$

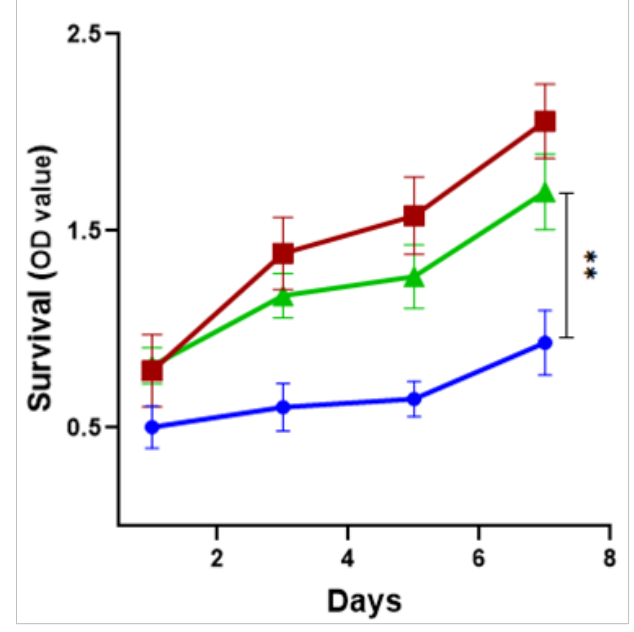

E

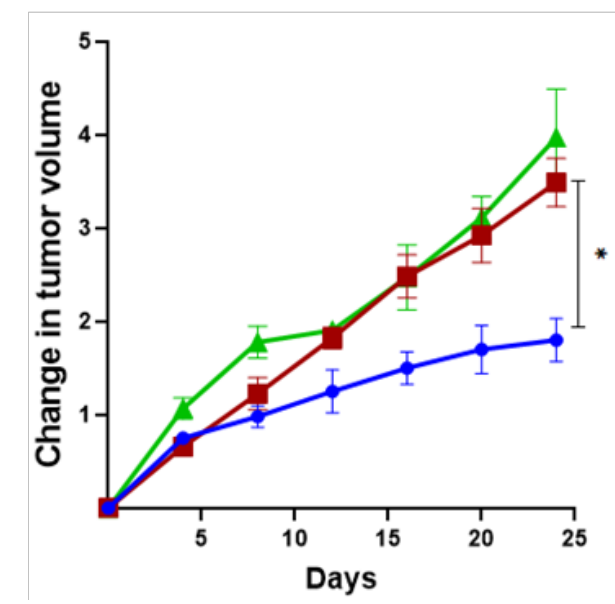

Figure 9. PIK3CA and AKT1 mutations confer growth advantage in vitro and in vivo. A)Cells were seeded at 500 cells/well in 96-well plates in triplicate and treated with 10\% FBS and B) $5 \%$ CSS supplemented with $5 \mathrm{nM} \beta$-estradiol. SRB assay were used to determine the viability of the cells every two days. C) Cells were seeded at 1,000 cells/well in 96-well plates in triplicate. Cell counting was used to determine the survival of the cells every two days. D) For in vivo growth of each genotype, six estrogen-supplemented nude mice per group were injected with $5 \times 10^{\wedge} 6$ cells suspended in matrigel. The tumor volumes were measured twice a week for 25 days. P-value calculated based on ANOVA: 0.1234 (ns), $0.0332\left(^{*}\right)$, $\left.\left.0.0021^{(* *}\right), 0.0002^{(* \star}\right)$ and $\left.<0.0001^{(* \star *}\right)$. 

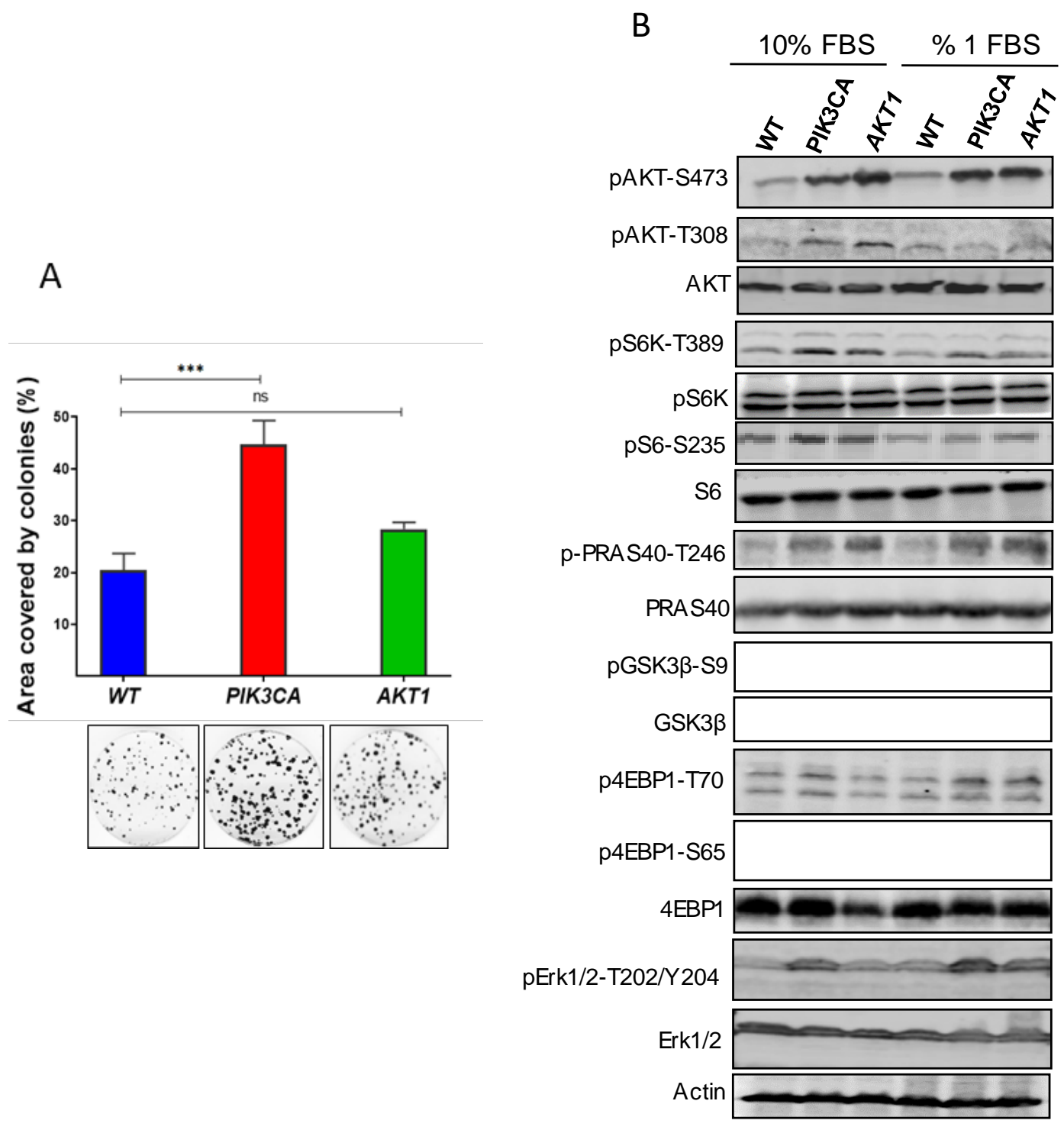

Figure 10. PI3K pathway signaling and colony formation ability in PIK3CA and AKT1 mutations.A) MCF7 isogenic cell lines were treated with five different concentrations of palbociclib and vehicle in a ten-fold serial dilution series for 72 hours. SRB assay was used to assess cell growth effect and the IC50 values were calculated using dose-effect analyzer software CalcuSyn 2.0. B) Cells were treated with increasing concentrations of palbociclib and vehicle for three weeks. The colonies were then fixed and stained with crystal violet. C) Quantification of crystal violet staining from cells in B using NIH ImageJ v.1.46 software. P-value calculated based on ANOVA: 0.1234 (ns), $0.0332\left(^{\star}\right), 0.0021\left(^{\star \star}\right), 0.0002\left(^{\star \star \star}\right)$ and $<$ $0.0001(* \star \star *)$. 


\subsubsection{PIK3CA and AKT1 mutations reduce the sensitivity of palbociclib in vitro}

We next examined whether PIK3CA or AKT1 mutations can impact the response to palbociclib. The CDK4/6 inhibitor palbociclib in combination with endocrine therapy has been approved by the FDA for the treatment of ER-positive, HER2-negative advanced breast cancer [93]. Cell growth assay and colony formation were performed to determine palbociclib efficacy in MCF7 cell lines with varying genotypes. Wild-type cells showed significant reduction in IC50 (Figure 11A) ( $p<0.0001$ for PIK3CA vs WT and $p=0.043$ for $A K T 1$ vs WT) and anchoragedependent proliferation shown by formation of fewer colonies in response to palbociclib treatment, but the presence of PIK3CA and AKT1 mutations conferred less sensitivity (Figures 11B and C).

It is known that CDK4/6 forms a complex with cyclin D to promote cell cycle progression through phosphorylation and inactivation of protein RB [134]; therefore, we examined the effect of CDK4/6 inhibition on cell cycle. Treatment with increasing doses of palbociclib resulted in a prominent decrease in RB phosphorylation (serine 807/811) in WT cells, while PIK3CA and AKT1 mutants had less decrease in pRBS807/811 (Figures 12A and B). Additionally, palbociclib led to a dramatic decrease in S and G2-M phases in WT cells, compared with mutants (Figures 12C and D) ( $p=0.015$ for palbociclib vs Ctrl. In WT). These observations indicate that PIK3CA and AKT1 mutations attenuate the efficacy of palbociclib. 


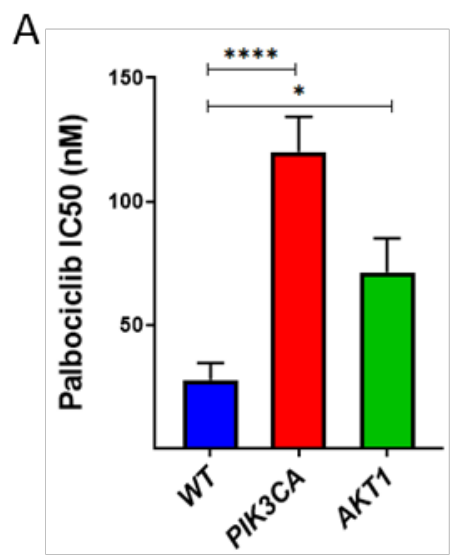

B

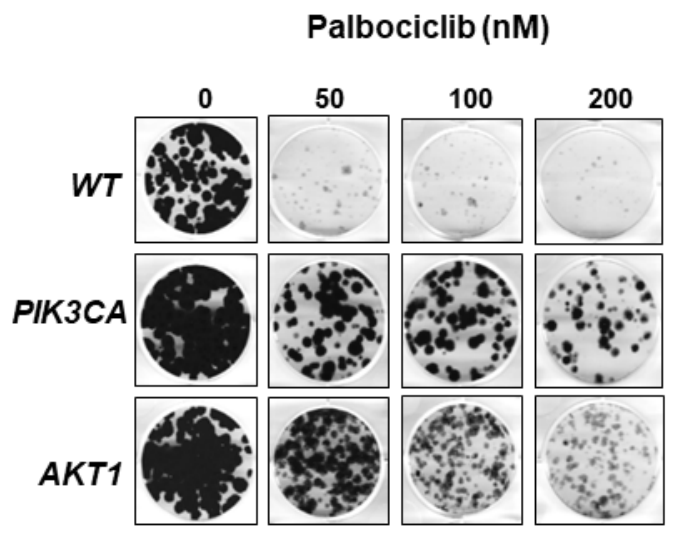

C

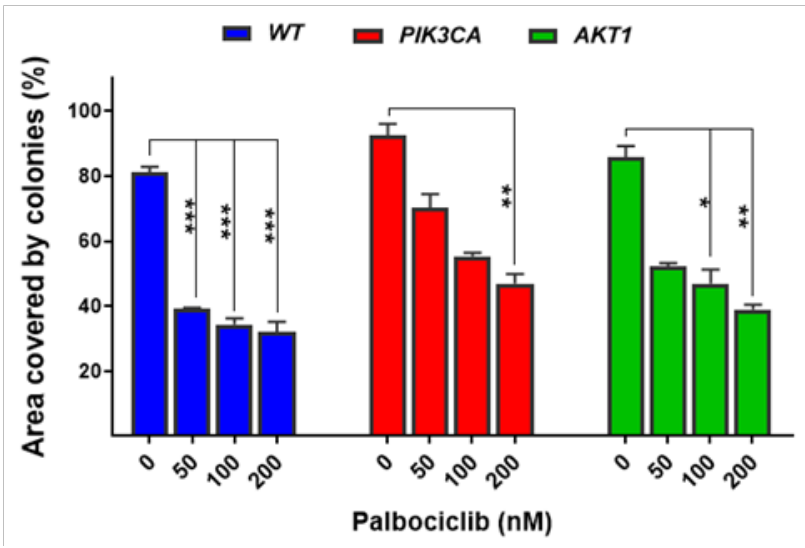

Figure 11. PIK3CA and AKT1 mutations show less sensitivity to palbociclib.A) Cells were seeded at 1,000 cells $/ 60 \mathrm{~mm}$ plates in triplicate. Colonies were then stained with crystal violet and counted using NIH ImageJ v.1.46 software after 3 weeks. B) Cells were grown in medium containing $10 \%$ and $1 \%$ FBS. Equal amounts of protein were loaded on SDS-PAGE gels and blotted with the antibodies shown. P-value calculated based on ANOVA: 0.1234 (ns), $0.0332\left({ }^{*}\right), 0.0021\left(^{(*}\right)$, $0.0002\left(^{* * *}\right)$ and $<0.0001(* * *)$. 


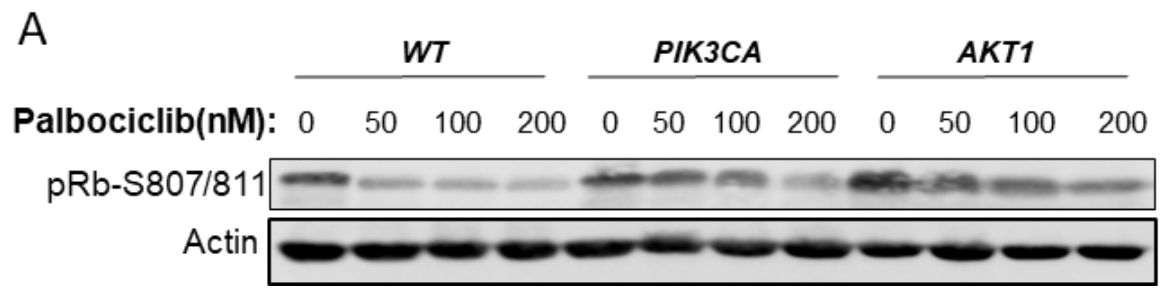

B
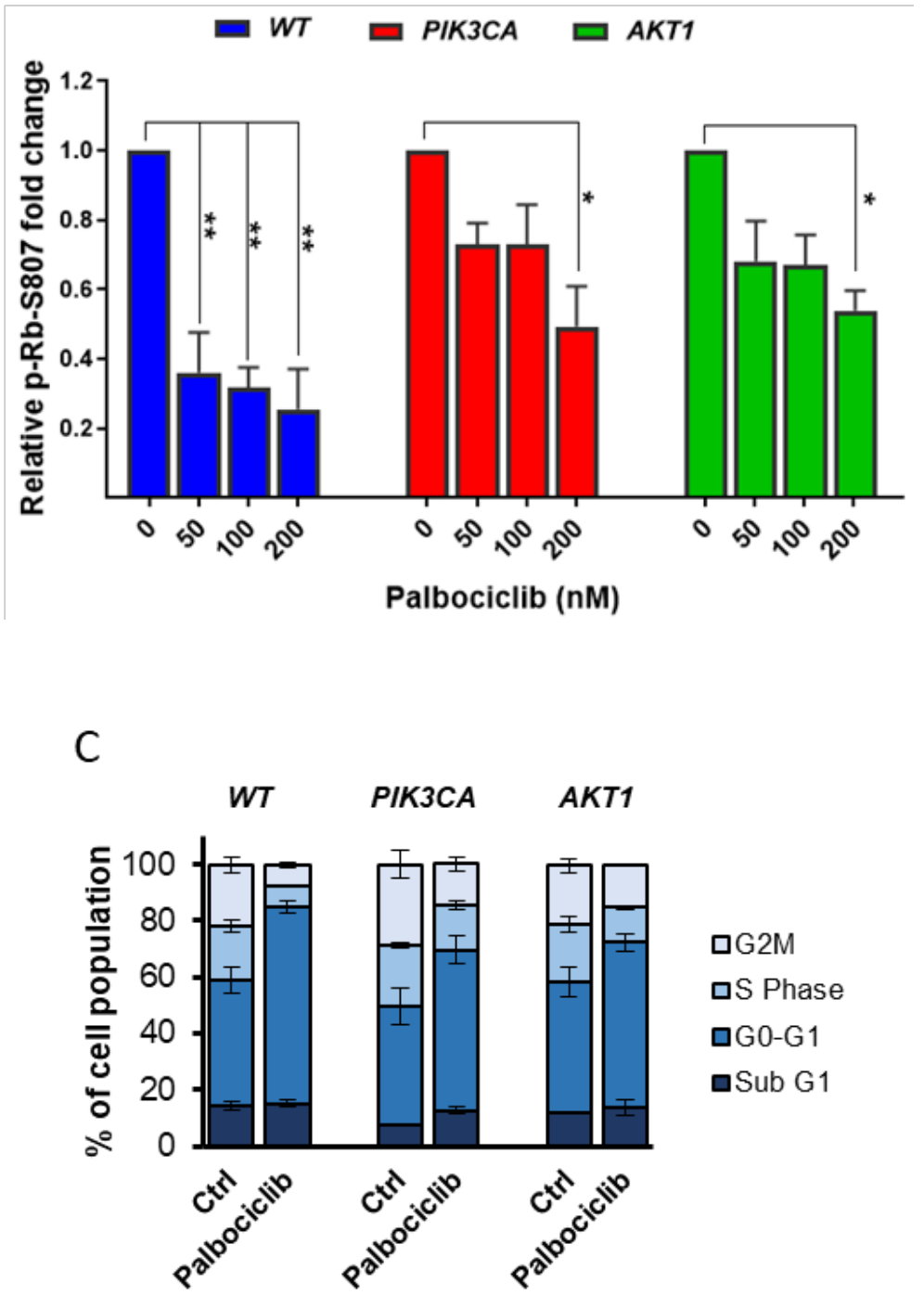


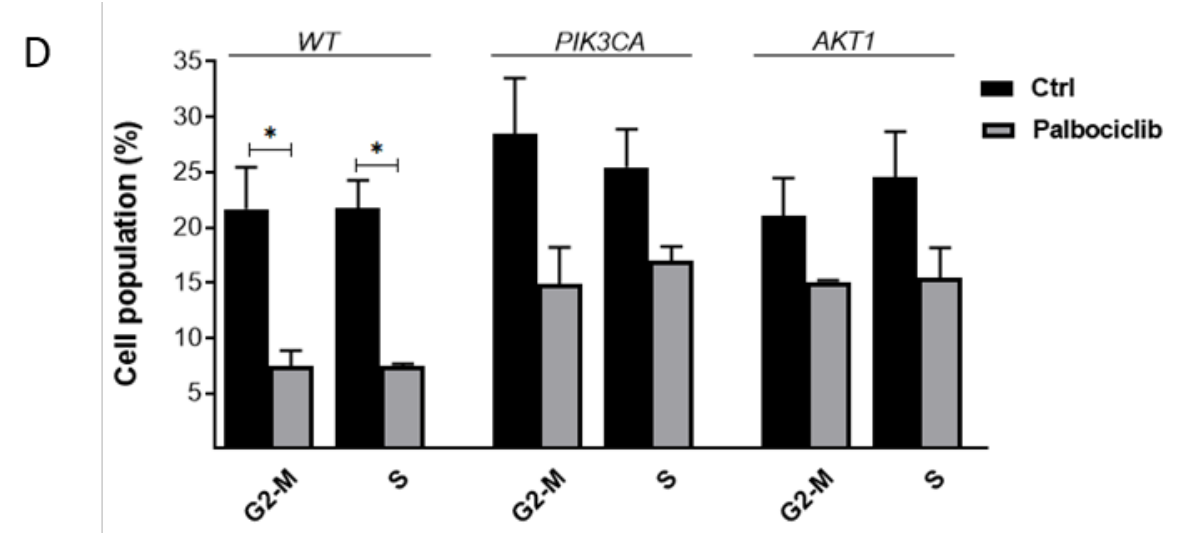

Figure 12. PIK3CA and AKT1 mutations modulate RB phosphorylation and cell cycle progression in response to palbociclib.A) The expression level of RB phosphorylation (serine 807/811) in cells treated with various concentrations of palbociclib for 24 hours was determined by western blotting. B) Densitometric quantification of western blot bands was analyzed with Image Studio Lite Ver 5.2. Protein levels were normalized to $\beta$-Actin. C) Cell were treated with $100 \mathrm{nM}$ of palbociclib for 24 hours. The cells were then fixed and stained with propidium iodide and the cell abundance in different phases of cell cycle were analyzed with flow cytometry. D) Bar graphs show the quantification analysis for the significance of changes in G2-M and S phases. All data mean \pm SD are indicated using triplicate samples of each representative experiment. P-value calculated based on ANOVA: $\left.0.1234(\mathrm{~ns}), 0.0332\left(^{(}\right), 0.0021\left(^{\star *}\right), 0.0002{ }^{(* \star}\right)$ and $\left.<0.0001{ }^{* * * *}\right)$.

\subsection{SUMMARY}

It has been noted by others that PI3K and MAPK signaling pathways crosstalk with each other[135]. Mutant PIK3CA led to a marked increase in MAPKregulated kinase ERK1/2 phosphorylation. However, the presence of AKT1-E17K in MCF7 cells with wild-type PIK3CA did not affect ERK1/2 phosphorylation level, suggesting that such interaction is cell type specific. Despite stimulation of PRAS40, PIK3CA and AKT1 did not induce phosphorylation of downstream targets, including p70S6K and ribosomal protein S6 (pS6). Overall, increased PI3K/AKT signaling activity was observed in the presence of full serum-more in mutant cell lines than 
wild-type MCF7—but it did not restore the phosphorylation level of downstream targets in MCF7-WT to the same level as found in mutant cells (Figure 10B).

The PISK/AKT pathway is one of the most frequently mutated signaling pathways in breast cancer and drives tumor survival, genomic instability, motility, and progression [115]. Alterations in the genes encoding several components of the PI3K/AKT pathway, such as mutations of PIK3CA and AKT1 genes, often occur in ER-positive breast cancer [136]. In our isogenic model system, PIK3CA and AKT1 conferred growth advantage and enhanced the in vivo tumorigenic properties of luminal breast cancer (Figures 9A-C). This data also suggest a bona fide oncogenic function for AKT1-E17K mutation. In lung epithelial cells, AKT1-E17K mutation has been shown to have an oncogenic role due partly to its involvement in CDK inhibitor p27 regulation [137]. Understanding the predictive biomarker value of specific PI3K pathway driver mutations can enhance the response to current and future novel targeted therapies in ER-positive luminal breast cancer.

The CDK4/6 inhibitors have profoundly transformed the therapeutic index of HR-positive, HER2-negative advanced breast cancer. They have provided survival benefits and substantial efficacy in combination with endocrine therapies. In the PALOMA-1 and PALOMA-2 trials, the addition of palbociclib enhanced median PFS, compared with letrozole alone $[100,138]$. Further, patients who received palbociclib with fulvestrant in PALOMA-3 had a longer PFS than those who were treated with fulvestrant plus placebo [97]. Despite the clinical benefits of CDK4/6 inhibitors in the treatment of ER-positive breast cancer, acquired resistance to CDK4/6 inhibition limits their efficacy. Ongoing preclinical studies are currently investigating various 
pathways potentially responsible for resistance. Given the heterogeneity nature of HR-positive breast cancer, exploring the mechanisms of resistance requires acknowledgment of the tumor's genomic background.

We demonstrated that PIK3CA and AKT1 mutant cell lines are less responsive to the cytotoxicity effect of palbociclib and exhibited higher IC50, compared with WT cells (Figure 11A). Exposure to different concentrations of palbociclib induced markedly greater inhibition of proliferation and colony formation in WT cells (Figures 11B and C). In addition, after treatment with palbociclib, the protein expression level of RB phosphorylation was remarkably decreased, whereas PIK3CA and AKT1 mutant cell lines had a lesser reduction (Figures 12A and B). More significant G2-M and S phase arrests were observed in WT than in mutant cell lines in response to palbociclib (Figures $12 \mathrm{C}$ and D). Taken together, these results showed that the presence of either PIK3CA or AKT1 mutation is sufficient enough to reduce palbociclib efficacy. This is an important finding that can help in addressing CDK4/6 inhibition resistance and optimize palbociclib use in the clinical treatment of ER-positive breast cancer. 


\section{CHAPTER 4: SYNTHETIC LETHALITY SCREENING IDENTIFIES DDR1 AS A}

SYNTHETIC LETHAL PARTNER OF PALBOCICLIB IN PIK3CA AND AKT1

\section{MUTANT ER-POSITIVE BREAST CANCER CELL LINES}

\subsection{INTRODUCTION}

The cross talk between CDK4/6 and PI3K pathways described in the literature provided a rationale for combined targeting of the CDK4/6-PI3K axis $[139,140]$. Likewise, the combination of CDK4/6 and PI3K inhibitors induced synergistic interaction, leading to greater tumor regression and improved outcome in PIK3CA mutant breast tumor [118, 121]. In preclinical and clinical studies, prolonged exposure to CDK4/6 inhibition provoked adaptive resistance in ER-positive breast cancer $[118,141]$. Despite numerous attempts to identify biomarkers responsible for 
resistance or predict response to CDK4/6 inhibitors, little has benefited the clinical outcomes of breast cancer.

Given the available preclinical and clinical data, the compensatory activation of the PI3K oncogenic pathway might be a relevant candidate to induce acquired resistance to CDK4/6 inhibition [118]. Meanwhile, previous results (Chapter 3) showed that the presence of PIK3CA and AKT1 in an isogenic model system decreased the in vitro efficacy of palbociclib. Therefore, the goal of this chapter was to identify ways to overcome palbociclib resistance induced by PIK3CA/AKT1. Given the frequent somatic mutations in the PI3K pathway, finding additional targets through identifying other potential survival pathways that take advantage of PIK3CA and AKT1 driving mutations will enhance the clinical benefit of palbociclib as well as the current therapy of ER-positive breast cancer.

We have used a human kinome shRNA library targeting a variety of cancerrelated kinases to determine potential targets that only affect the survival in cells with PIK3CA or AKT1 mutations when treated with palbociclib. Further, we validated the candidate genes to identify the most effective combinational partner to enhance palbociclib efficacy in cells harboring PIK3CA and $A K T 1$ mutations. The synthetic lethality experiment can help us design rational combination therapies that take advantage of $P I K 3 C A$ and $A K T 1$ driving mutations to be used in clinical trials for confirming antitumor effects. 


\subsection{RESULTS}

\subsubsection{Using a pooled shRNA library and dropout selection screening to identify potential synthetic lethal genes in response to palbociclib}

To determine ways for improving the response of ER-positive breast cells to CDK4/6 inhibition, we conducted a synthetic lethality screen in MCF7 cells with wildtype or mutant PIK3CA or AKT1 mutant status. For this we used a lentiviral library consisting of 5,500 barcoded shRNA constructs, targeting 530 cancer-related human kinases. Because different cancer cell lines exhibit differential sensitivity to lentiviral transduction, we determined the $\mathrm{MOI}$ required to transduce $20 \%$ of the cells (Figure 7). When the number of infected cells is at or below $20 \%$, each transduced cell expresses a single shRNA with good approximation. The transduced cells were treated with palbociclib for 2 weeks before isolating the genomic DNA (gDNA) to analyze the sequence of integrated shRNAs. A pellet of pooled cells for each cell line was frozen to be used as a reference for confirming equal representation of each shRNA before starting palbociclib treatment (Figure 13A).

Negative screen analysis was performed by high-throughput quantitative RTPCR, and a gene summary was generated by using redundant shRNA activity (RSA) scoring, which provides a ranking of each gene in the screen. Using log 10 p-value of $0.05(-1.3)$ as the cut-off point to define significant hits, we compared the top scoring genes in the presence of drug to vehicle-treated cells to identify target genes that provide a selective disadvantage in the presence of palbociclib (Figures 13B and Table 4). 
We prioritized the top identified hits in each group on the basis of prognostic and therapeutic implications and the clinical relevance of genes in breast cancer. In addition, we employed previous knowledge of the associations of kinases with ER status and functional role in the PISK/AKT pathway to select the top potential synthetic lethal targets that were mainly involved in cell cycle regulation and proliferation pathways (Table 5).

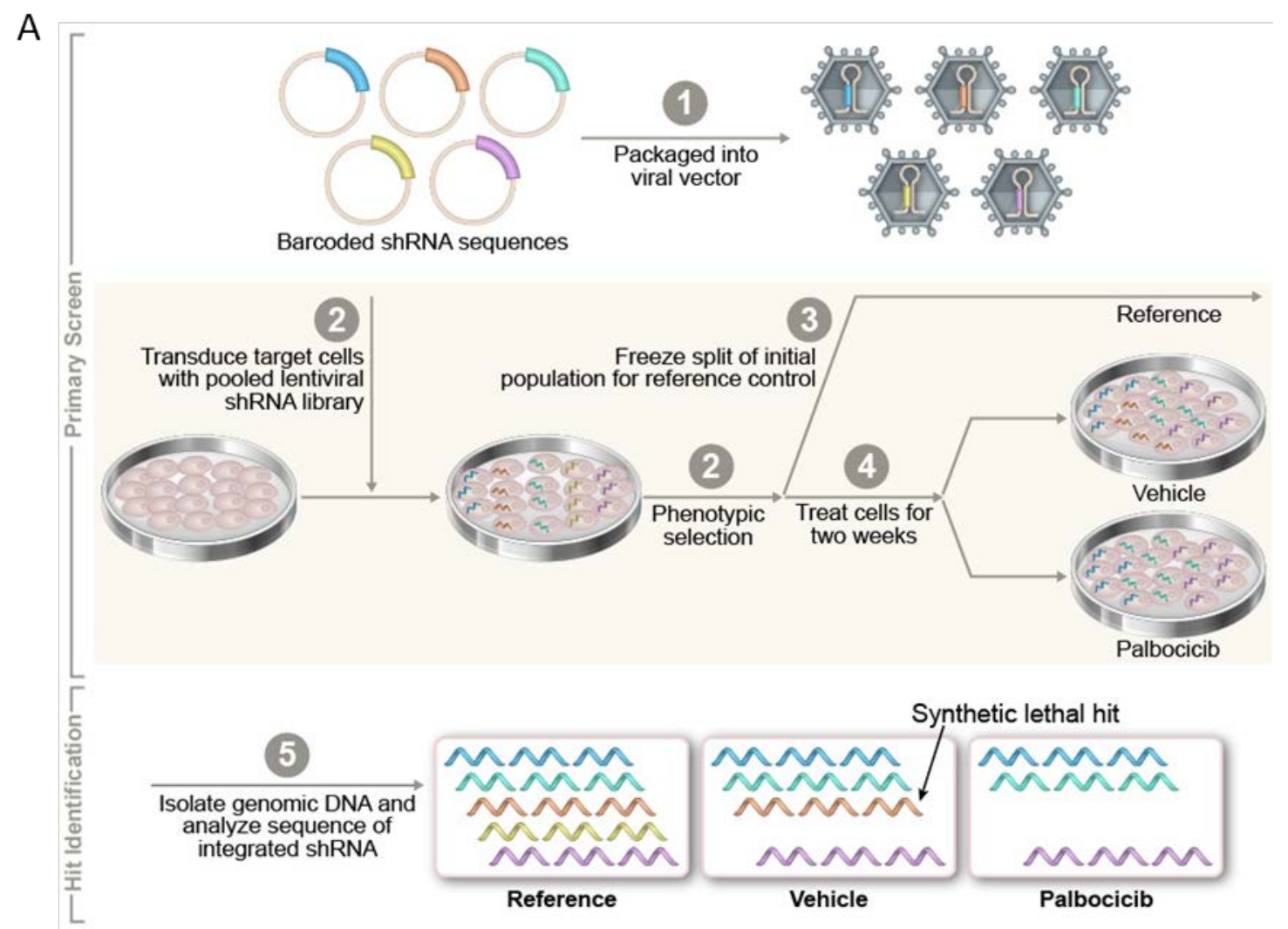


B
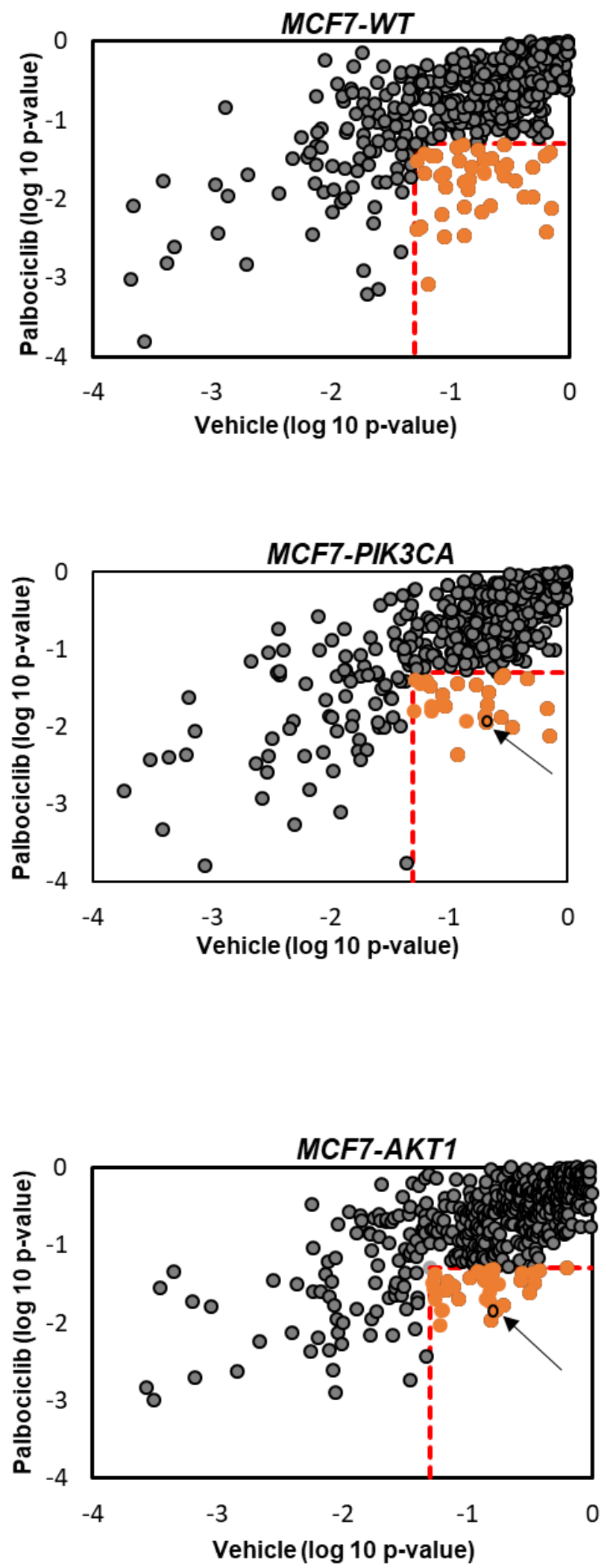
Figure 13. Transduction of MCF7 cell lines with lentiviral shRNA library and negative selection screeningA) Schematic depiction of the pooled ShRNA screening workflow. MCF7 isogenic cells were transduced with human kinome lentiviral library consists of 5500 barcoded shRNA constructs targeting 530 human kinases and treated with palbociclib in addition to vehicle for two weeks. B) Negative screen analysis was assessed for the indicated cell lines by high-throughput quantitative RT-PCR to create gene summary using redundant shRNA activity scoring (RSA). The orange dots indicate top scoring hits in the presence of palbociclib treatment based on -1.3 cut-off point ( $\log 10$ of 0.05$)$. DDR1, a common top hit in PIK3CA and AKT1, is distinguished with an arrow. 


\begin{tabular}{|c|c|c|}
\hline \multicolumn{3}{|c|}{ MCF7-WT } \\
\hline Gene name & $\begin{array}{l}\text { RSA Score } \\
\left(\log _{10} P_{\text {Value }}\right)\end{array}$ & Rank \\
\hline MAPK15 & -3.07 & 5 \\
\hline$C D K 8$ & -2.48 & 12 \\
\hline MTOR & -2.47 & 13 \\
\hline MAST3 & -2.42 & 17 \\
\hline MAP3К12 & -2.38 & 18 \\
\hline МАРКб & -2.35 & 19 \\
\hline MAPK12 & -2.20 & 23 \\
\hline STK31 & -2.17 & 24 \\
\hline$D A P K 2$ & -2.12 & 26 \\
\hline$C D K L 2$ & -2.11 & 27 \\
\hline PRKCD & -2.08 & 30 \\
\hline NTRK1 & -1.98 & 34 \\
\hline$A C V R 2 A$ & -1.97 & 36 \\
\hline HIPK1 & -1.88 & 42 \\
\hline$T N K 1$ & -1.85 & 44 \\
\hline PLK2 & -1.79 & 48 \\
\hline ULK2 & -1.77 & 51 \\
\hline EPHAS & -1.75 & 53 \\
\hline AURKA & -1.71 & 56 \\
\hline HSPB8 & -1.71 & 57 \\
\hline$P I K 3 C D$ & -1.68 & 60 \\
\hline TSSK4 & -1.68 & 61 \\
\hline PRKAB1 & -1.67 & 62 \\
\hline EPHA10 & -1.65 & 63 \\
\hline RPSGKAG & -1.62 & 65 \\
\hline ITK & -1.60 & 68 \\
\hline$A D C K 1$ & -1.60 & 69 \\
\hline TTBK1 & -1.56 & 71 \\
\hline RPS6KL1 & -1.52 & 75 \\
\hline MAP2K才 & -1.51 & 76 \\
\hline NUAK2 & -1.51 & 77 \\
\hline CSNK1G2 & -1.49 & 79 \\
\hline
\end{tabular}




\begin{tabular}{|c|c|c|}
\hline \multicolumn{3}{|c|}{ MCF7-P1K3CA-E545K } \\
\hline Gene name & $\begin{array}{l}\text { RSA Score } \\
\left(\log _{10} P_{\text {Value }}\right)\end{array}$ & Rank \\
\hline NEK8 & -2.36 & 20 \\
\hline ADCK5 & -2.12 & 27 \\
\hline STK31 & -2.00 & 32 \\
\hline MAP4K1 & -1.95 & 36 \\
\hline$D D R 1$ & -1.93 & 38 \\
\hline STRADA & -1.88 & 41 \\
\hline TSSK4 & -1.86 & 46 \\
\hline PIK3CD & -1.80 & 47 \\
\hline PRKACA & -1.79 & 48 \\
\hline МАР $3 К 15$ & -1.77 & 50 \\
\hline CASK & -1.74 & 52 \\
\hline$U L K 1$ & -1.73 & 53 \\
\hline CSNK1G3 & -1.72 & 54 \\
\hline EIF2AK2 & -1.71 & 55 \\
\hline MAPK10 & -1.59 & 64 \\
\hline TESK1 & -1.56 & 65 \\
\hline$S G K 2$ & -1.48 & 67 \\
\hline SIK1 & -1.46 & 71 \\
\hline МҮОЗВ & -1.45 & 73 \\
\hline ADCK3 & -1.44 & 75 \\
\hline PAK6 & -1.41 & 78 \\
\hline CNKSR1 & -1.40 & 81 \\
\hline CSNK1A1L & -1.37 & 82 \\
\hline STK19 & -1.36 & 86 \\
\hline
\end{tabular}




\begin{tabular}{|c|c|c|}
\hline \multicolumn{3}{|c|}{ MCF7-AKT1-E17K } \\
\hline Gene name & $\begin{array}{l}\text { RSA Score } \\
\left(\log _{10} P_{\text {Value }}\right)\end{array}$ & Rank \\
\hline $\mathrm{HCK}$ & -2.02 & 26 \\
\hline RPS6KL1 & -1.97 & 28 \\
\hline$T L K 2$ & -1.84 & 33 \\
\hline PRKCB & -1.84 & 34 \\
\hline CDK20 & -1.84 & 35 \\
\hline$D D R 1$ & -1.77 & 41 \\
\hline MAP2K2 & -1.73 & 44 \\
\hline MAP2K6 & -1.70 & 45 \\
\hline ANKK1 & -1.69 & 47 \\
\hline MAP3К15 & -1.69 & 48 \\
\hline МАРЗК9 & -1.62 & 56 \\
\hline AKT1 & -1.60 & 57 \\
\hline SRPK2 & -1.58 & 60 \\
\hline PDPK1 & -1.57 & 61 \\
\hline$F R K$ & -1.56 & 62 \\
\hline EPHA5 & -1.54 & 66 \\
\hline ATR & -1.50 & 70 \\
\hline FGFR2 & -1.48 & 72 \\
\hline LATS1 & -1.48 & 75 \\
\hline PRKCH & -1.47 & 77 \\
\hline$P R K C D$ & -1.45 & 80 \\
\hline$A C V R 2 A$ & -1.45 & 81 \\
\hline МҮОзВ & -1.41 & 83 \\
\hline KIT & -1.38 & 84 \\
\hline FLJ40852 & -1.36 & 87 \\
\hline$A L K$ & -1.35 & 90 \\
\hline PRKCE & -1.35 & 91 \\
\hline IRAK4 & -1.35 & 92 \\
\hline$P D K 1$ & -1.34 & 94 \\
\hline CDK4 & -1.33 & 95 \\
\hline
\end{tabular}

Table 4. List of the candidate genes that confer a selective disadvantage in the presence of palbociclib.Negative screen analysis was assessed by highthroughput quantitative RT-PCR using the MCF7 isogenic cell lines transduced with hKinome lentiviral library. The identified candidate synthetic lethal genes in the presence of palbociclib treatment using p-value $<0.05$ are listed for WT (wild-type), PIK3CA-E545K, and AKT1-E17K cell lines. 


\begin{tabular}{l|l|c|l}
\multicolumn{1}{c|}{ Gene } & $\begin{array}{c}\text { Synthetic lethal } \\
\text { partner }\end{array}$ & RSA score (-) & Function \\
\hline DDR1 & PIK3CA/AKT1 & $1.9 / 1.8$ & Migration and invasion. \\
MTOR & WT & 2.47 & Cell growth, proliferation, motility and survival \\
NEK8 & PIK3CA & 2.36 & Cell cycle progression \\
PRKCD & AKT1MT & $2 / 1.68$ & Regulation of growth and apoptosis \\
STK31 & PIK3CANT & $2 / 2.17$ & Cell cycle regulated \\
TLK2 & AKT1 & 1.84 & Apoptosis pathway and inhibition of $\mathrm{G} / \mathrm{M}$
\end{tabular}

Table 5. List of the selected potential synthetic lethal genes for palbociclib treatment. Genes were prioritized on the basis of their functional and clinical associations in breast cancer.

\subsubsection{Synthetic lethality screening identifies $D D R 1$ as a synthetic lethal partner of palbociclib in PIK3CA and AKT1 mutant ER-positive breast cancer cell lines}

Using shRNA knockdown and SRB proliferation assay, we examined the candidate hits, including DDR1, MTOR, NEK8, PRKCD, STK31, and TLK2. The results indicated that silencing $D D R 1$ by using two different shRNAs inhibited the growth of PIK3CA and AKT1 mutant and wild-type ER-positive cell lines in vitro (Figure 14A). However, the mutant DDR1 knockout cells were more sensitive to the inhibitory effect of palbociclib, showing that DDR1 silencing selectively sensitized PIK3CA and AKT1 mutant cells to palbociclib (Figure 14B). 
A

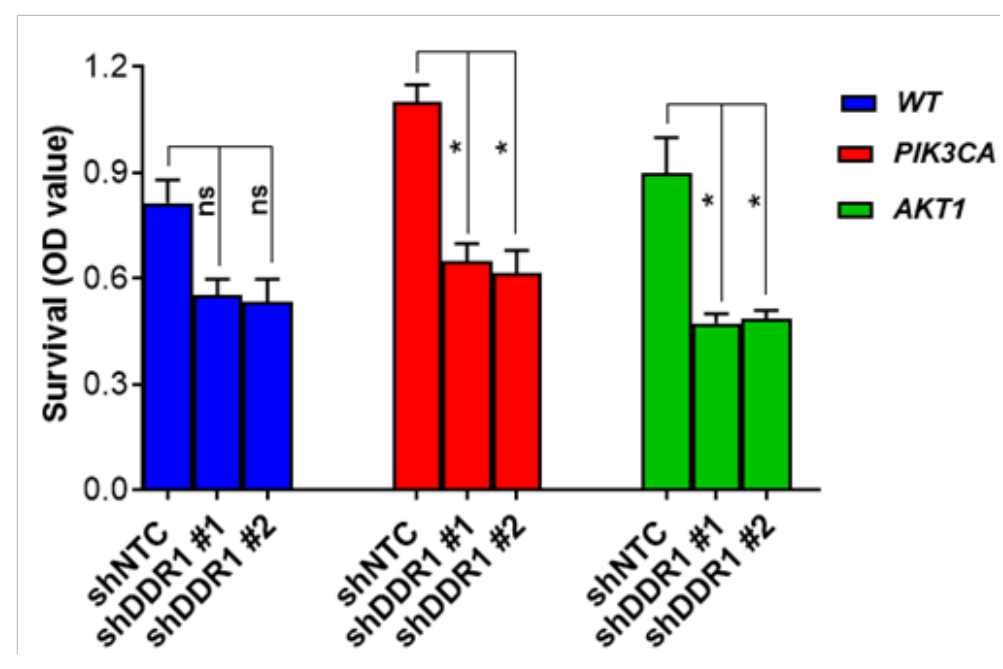

B

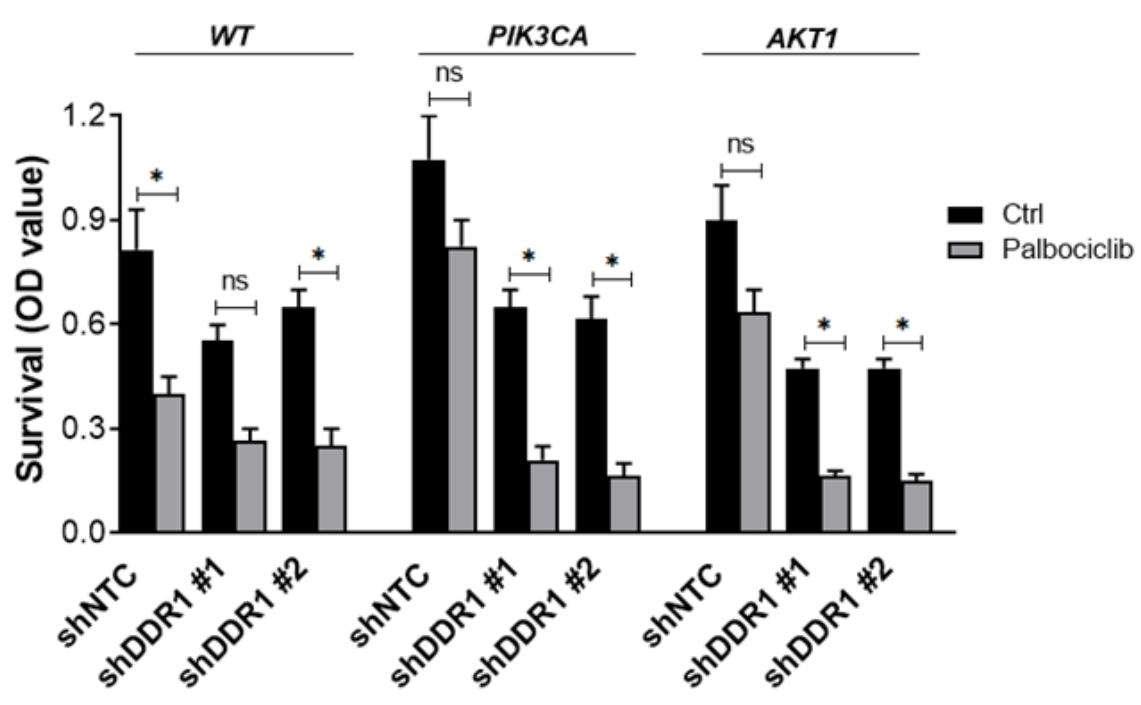

Figure 14. shRNA library screening identifies DDR1 as a candidate synthetic lethal partner for palbociclib in both PIK3CA and AKT1 mutant cell linesA) Cells were infected with non-targeting control shRNA (shRNA-NTC) and two different shRNAs targeting DDR1. Following 48 hours puromycin selection, cells were seeded at 1,000 cells/well in 96-well plates in triplicate. SRB assay was used to determine the viability of the cells every two days. B) The puromycin selected cells in A were treated with vehicle (DMSO) or $100 \mathrm{nM}$ palbociclib for 72 hours. Cell survival was analyzed using SRB colorimetric assay. All data mean \pm SD are indicated using triplicate samples of each representative experiment. P-value calculated based on ANOVA: $0.1234(\mathrm{~ns}), 0.0332\left(^{\star}\right), 0.0021\left(^{(*}\right), 0.0002\left(^{(\star \star}\right)$ and $<$ $\left.0.00011^{(\star \star \star}\right)$. 


\subsubsection{Correlation between DDR1 expression and ER status in a cohort of breast cancer patients}

To determine the correlation between DDR1 and ER expression level, we analyzed a cohort of breast cancer patients from The cancer Genome Atlas (TCGA), and Molecular Taxonomy of Breast Cancer International Consortium (METABRIC) datasets. The analysis showed that DDR1 mRNA level is significantly higher in ERpositive versus ER-negative tumors ( $p=4.7 \mathrm{e}-09$ for TCGA and $p=1 \mathrm{e}-19$ for METABRIC) (Figures 15A and B). In addition, 56\% (256/455 in TCGA) and 57\% (492/859 in METABRIC) of ER-positive samples were high-DDR1 tumors, whereas $33 \%$ (54/164 in TCGA) and 28\% (79/2282 in METABRIC) of ER-negative tumors had high-DDR1 expression, indicating that DDR1-high tumors are more frequent in ER-positive patients ( $p=1.99 \mathrm{e}-07$ for TCGA and $p=2.2 \mathrm{e}-16$ for METABRIC) (Figures $15 C$ and $D)$.

To further corroborate this finding, we interrogated data sets for different breast cancer subtypes and observed that HR-positive patients had higher DDR1 expression than did patients with TNBC ( $p=1.5 \mathrm{e}-05$ for TCGA and $p=2.5 \mathrm{e}-19$ for METABRIC) (Figures 16A and B). The tendency toward co-occurrence of highDDR1 expression and PIK3CA/AKT1 mutations in ER-positive patients was not consistent across different datasets, whereas AKT1 mutation occurs at a low frequency (Figures 16C-E). 
A

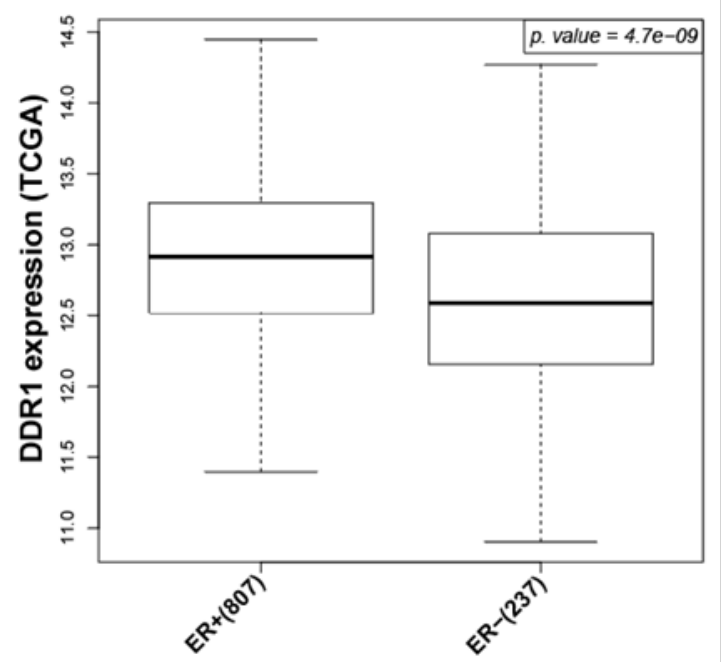

C

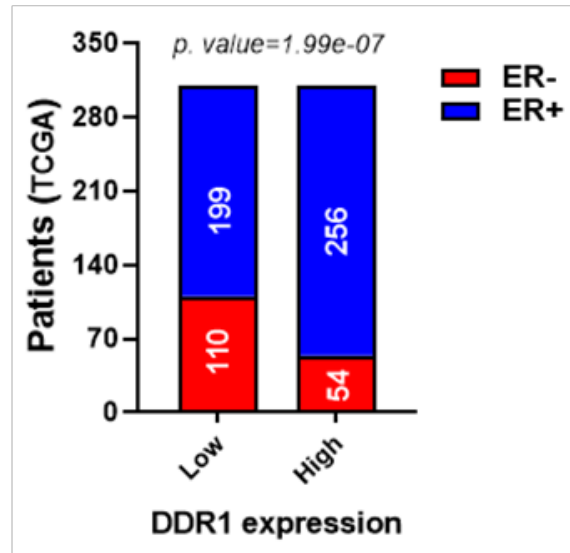

B

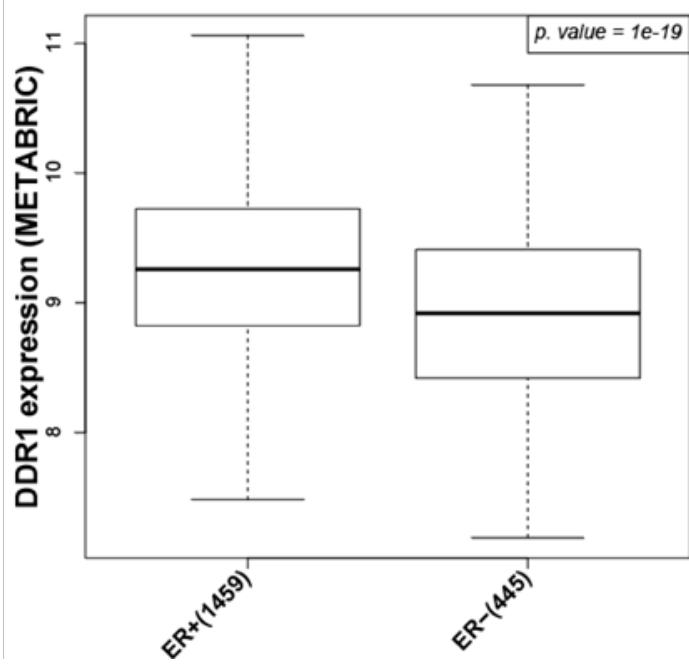

D

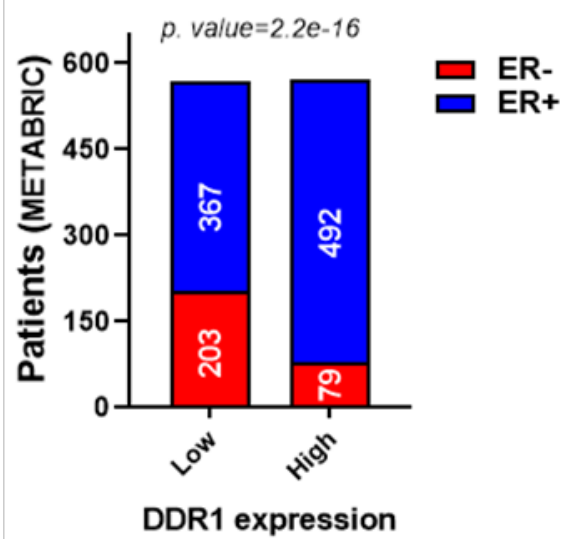

Figure 15. The correlation between DDR1 expression and ER status, in a cohort of breast cancer patients using TCGA and METABRIC dataset.A-B) RNA-seq. expression data was used to determine the level of DDR1 expression in ER-positive and ER-negative patients in TCGA (A) and METABRIC (B) dataset C-D) The Box and whisker plots show the frequency of DDR1 expression in ER-positive and ER-negative patients using the RNA-seq. expression data in TCGA (C) and METABRIC (D) datasets. Student's t-test was used to examine if the mean of the two groups were significantly different. The whiskers indicate the max and min values. The samples were arranged by the expression of DDR1 from high to low. The top 30\% samples are classified as "high", and the bottom 30\% samples are classified as "low". Fisher's Exact test was used to examine the frequency of 
high/low DDR1 expressed samples in patients based on ER-status. Student's t-test was used to examine if the mean of the two groups were significantly different. The whiskers indicate the max and min values.

A

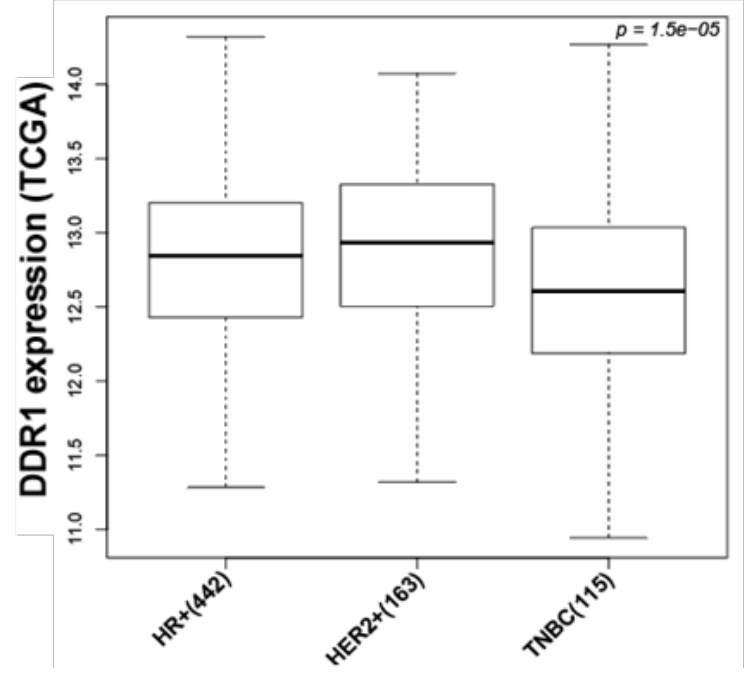

C

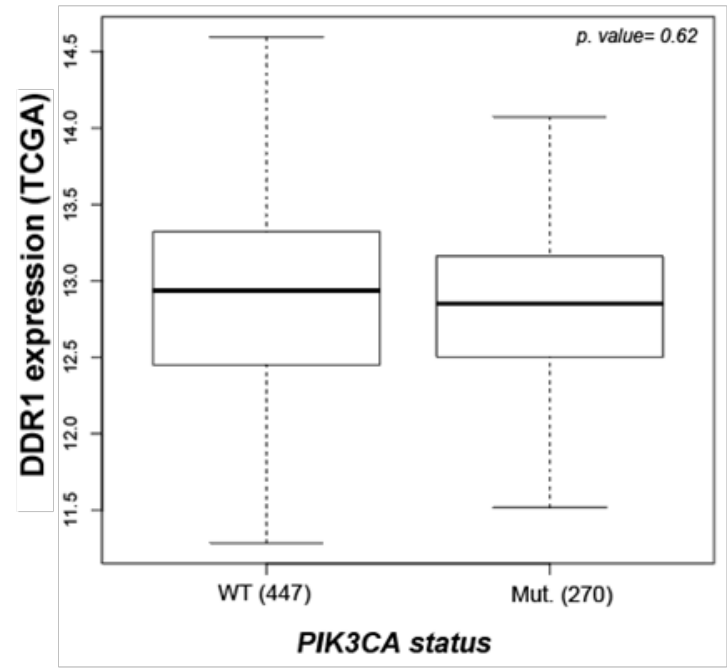

B

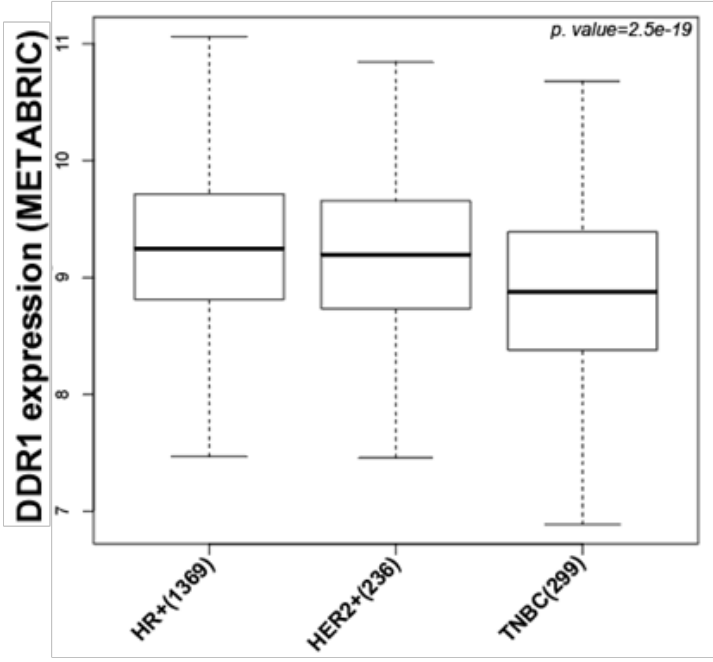

D

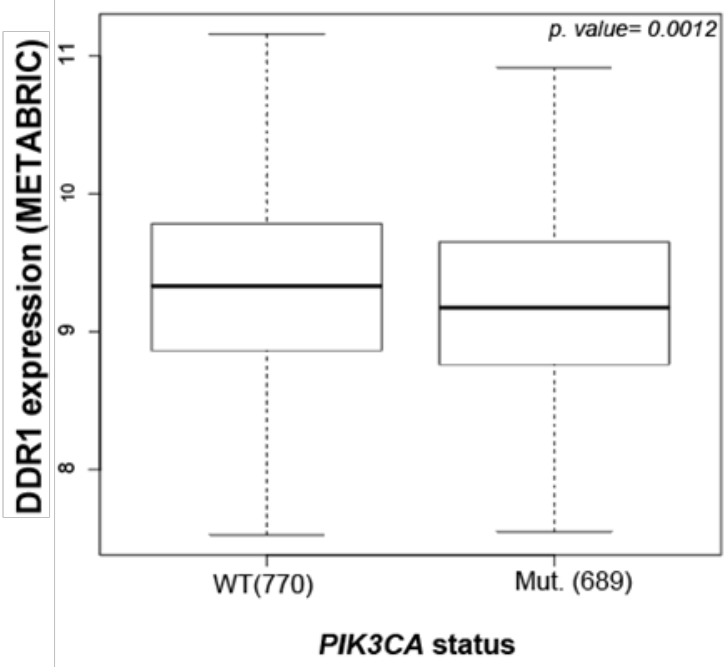




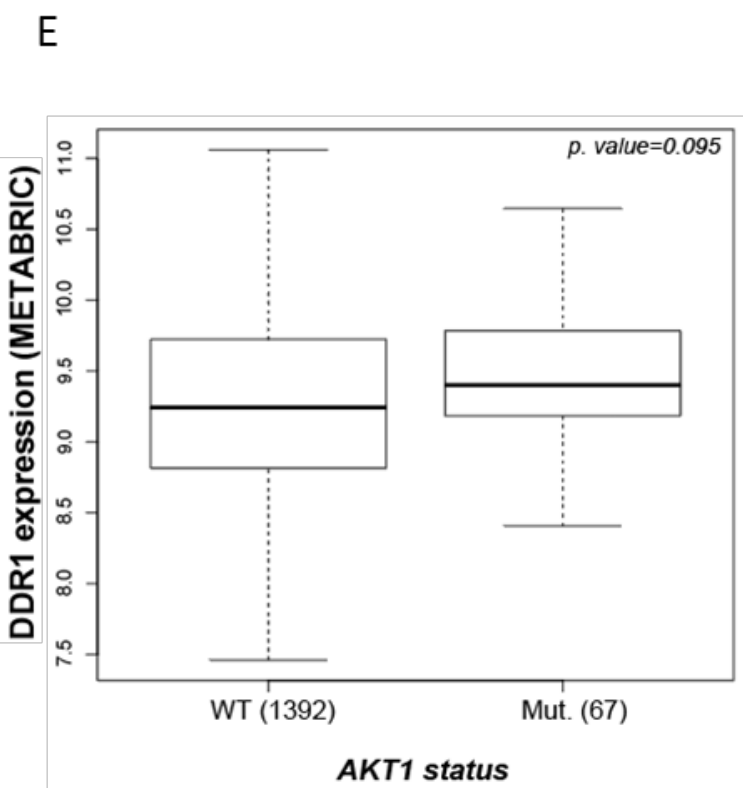

Figure 16. The expression of DDR1 in different breast cancer subtypes and ERpositive group with PIK3CA/AKT1 mutations using TCGA and MRTABRIC dataset. A-B) One-way ANOVA was used to determine whether there are any statistically significant differences between the means of HR-positive, HER2-positive and TNBC groups. C-E) Figures showing the expression of DDR1 in ER-positive patients with or without PIK3CA or AKT1 mutations. Student's t-test was performed to calculate the $\mathrm{p}$-values. There was only one ER-positive patient with AKT1 mutation in TCGA data set, so the comparison was excluded.

\subsection{SUMMARY}

On the basis of the screening results, we identified and functionally validated that discoidin domain receptor 1 (DDR1) is required for the survival and proliferation of PIK3CA and AKT1 mutant cells and selectively sensitizes these cells to palbociclib. DDR1, a collagen-activated tyrosine kinase, is amplified in various types of tumor, including $14 \%$ of metastatic breast cancers and has a critical role in cell attachment, survival, and proliferation [142]. Moreover, recent data suggest that DDR1 functions as a regulator of cell response to hormones, growth factors, and 
extracellular signals in breast cancer [143]. In fact, our analysis of breast cancer patients from the TCGA and METABRIC datasets showed that DDR1 expression level is significantly higher in ER-positive tumors than in TNBC tumors. In this study, we have shown that $D D R 1$ depletion inhibited cell proliferation and decreased clonogenicity while targeting DDR1 suppressed tumor growth. These findings support the potential role of $D D R 1$ as a therapeutic target for the treatment of ERpositive breast cancer associated with PI3K pathway activation.

CHAPTER 5: DDR1 SUPPRESSION INHIBITS PROLIFERATION AND SELECTIVELY SENSITIZES PIK3CA/AKT1 MUTANT CELLS TO PALBOCICLIB TREATMENT

\subsection{INTRODUCTION}

\subsubsection{Structure, activation, and function of DDR1}

Discoidin domain receptors (DDRs) are type I transmembrane RTKs that, unlike other RTKs, are specifically activated in response to collagens, a major component of the extracellular matrix (ECM) [144]. DDR1 and DDR2 are the two 
types of DDR kinases that display distinct-collagen binding specificity. DDR1 preferentially binds to collagens type $\mathrm{I}-\mathrm{VI}$ and VIII, whereas DDR2 is activated by collagens type I, III, and X [145-147]. The human DDR1 gene is located on chromosome 6 (6p21.3) and encodes five different DDR1 isoforms as a result of alternative splicing. DDR1a, DDR1b, and DDR1c are full-length receptors with functional enzymatic activity, whereas DDR1d and DDR1e are inactive kinases due to frame-shift and truncation genomic alterations [148]. The DDR2 gene maps to chromosome 1 and generates a single isoform of the receptor. DDR1 is composed of various structural domains, including: the $\mathrm{N}$-terminal discoidin (DS) domain, DSlike domain, extracellular juxtamembrane region (EJXM), transmembrane segment (TM), intracellular juxtamembrane region (IJXM), kinase domain (KD), and a short Cterminal domain at the very end (Figure 17) $[149,150]$.

DDR1 is involved in the regulation of mammary gland development and plays a role in cellular processes such as survival, proliferation, migration, invasion, matrix remodeling, and EMT [151-154]. After collagen binding, the cytosolic kinase domain of DDR1 becomes phosphorylated at several residues, leading to downstream signaling activation [155]. Collagen-induced DDR1 phosphorylation occurs several hours after ligand stimulation and remains active for as long as a day, showing significantly slow kinetics compared with other RTKs $[145,156]$. Phosphorylated sites in the kinase domain are able to bind to a number of adopter proteins, such as Src homology 2 (SH2) domain containing protein, [157], focal adhesion kinase (FAK) [158], C-terminal Src kinase (Csk) [159], p85 subunit of PI3K [160, 161] and prolinerich tyrosine kinase (pyk2) [158]. Some proteins, like Notch1 [162] and E-cadherin 
[163], are able to interact with DDR1 regardless of its collagen-induced phosphorylation. In mouse embryonic stem cells (mESCs), collagen I binds to and activates both DDR1 and $\alpha 2 \beta 1$ integrin. DDR1 induces Ras, PI3K/AKT, and ERK signaling cascade, while $\alpha 2 \beta 1$ integrin triggers Notch/Gli-1 upregulation. These events consequently activate Bmi-1 leading to RB inhibition and cell cycle progression in mESCs [164].

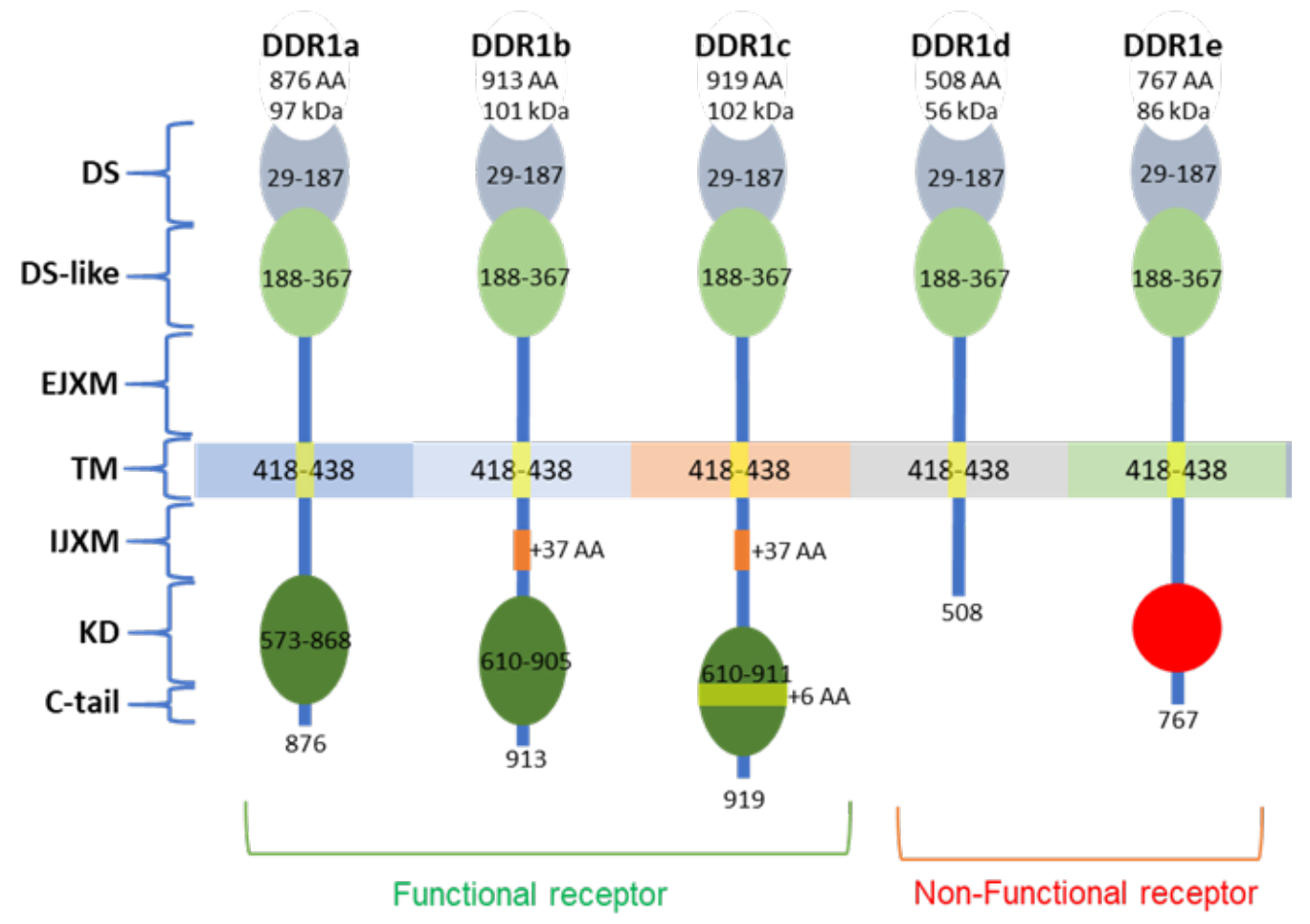

Figure 17. Structural domains of five different DDR1 isoforms. DDR1a, DDR1b and DDR1c are active kinase receptors. DDR1d and DDR1e are kinase deficient receptors. DS: discoidin domain; DS-like: discoidin-like domain; EJXM: extracellular juxtamembrane region; TM: transmembrane segment; IJXM: intracellular juxtamembrane region; KD: kinase domain; C-tail: short C-terminal domain. 


\subsubsection{DDR1 as a target in breast cancer}

Aberrant expression of DDR1 has been shown to promote tumor growth in various human cancers, including breast, lung, gastric, glioma, and ovary [165-168]. In breast cancer, DDR1 has been shown to promote invasion and survival and to regulate EMT and apoptosis in a microenvironment-dependent manner $[160,169$, 170]. In KRAS-mutant lung adenocarcinoma, the co-inhibition of DDR1 and Notch enhanced tumor growth suppression [165], whereas high DDR1 expression served as a survival factor in p53 wild-type breast cancer cells [152]. These evidences suggest that DDR1 has a complex role in breast cancer functions, as either an antiproliferating or pro-survival factor.

The differential effects of DDR1 expression in breast cancer may be impacted by the presence of other genomic alterations and hormonal receptor status. Collagen is the most abundant protein in $\mathrm{ECM}$, and the unique interaction between DDR1 and collagen plays an important role in tumor progression. Given the considerable gap in our knowledge of DDR1 functional behavior in breast cancer, more context-dependent investigation is required to understand DDR1 contribution to breast cancer progression. In the previous chapter, DDR1 was identified as a potential synthetic lethal partner that differentially enhanced palbociclib sensitivity in PIK3CA and AKT1 mutant cells. We also reported a higher DDR1 mRNA expression in luminal ER-positive breast tissues. Therefore, DDR1 expression and its proliferation effects need to be addressed to further confirm its selective action in our isogenic model system. 


\subsection{RESULTS}

\subsubsection{Silencing DDR1 with different targeted ShRNAs, and validating knockdown efficiency}

Numerous studies have shown the pro-survival role of DDR1 in breast cancer $[169,171]$. DDR1 has been implicated in the induction of EMT and functionally interacts with growth signaling receptors to positively modulate invasion and survival $[169,172,173]$. These results indicate that DDR1 is an important target for breast cancer and has a wide role in regulating cell response to growth factors and hormones. Therefore, to verify the DDR1 selective requirement in PIK3CAVAKT1 mutant cells, we analyzed the effect of suppressing $D D R 1$ and palbociclib treatment on cell viability and proliferation. Cells were infected with non-targeting control shRNA and three different DDR1-targeting shRNAs. After knockdown, efficiency was confirmed by using immunoblot and qRT-PCR (Figures 18A and B). Interestingly, the DDR1 protein and mRNA levels were higher in PIK3CAVAKT1 mutant cells, suggesting a correlation between DDR1 expression and palbociclib sensitivity (Figures $18 \mathrm{~A}$ and C).

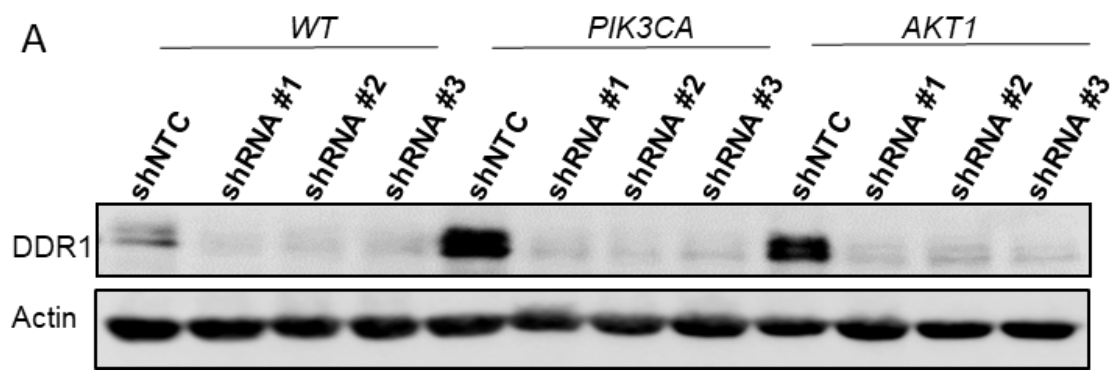




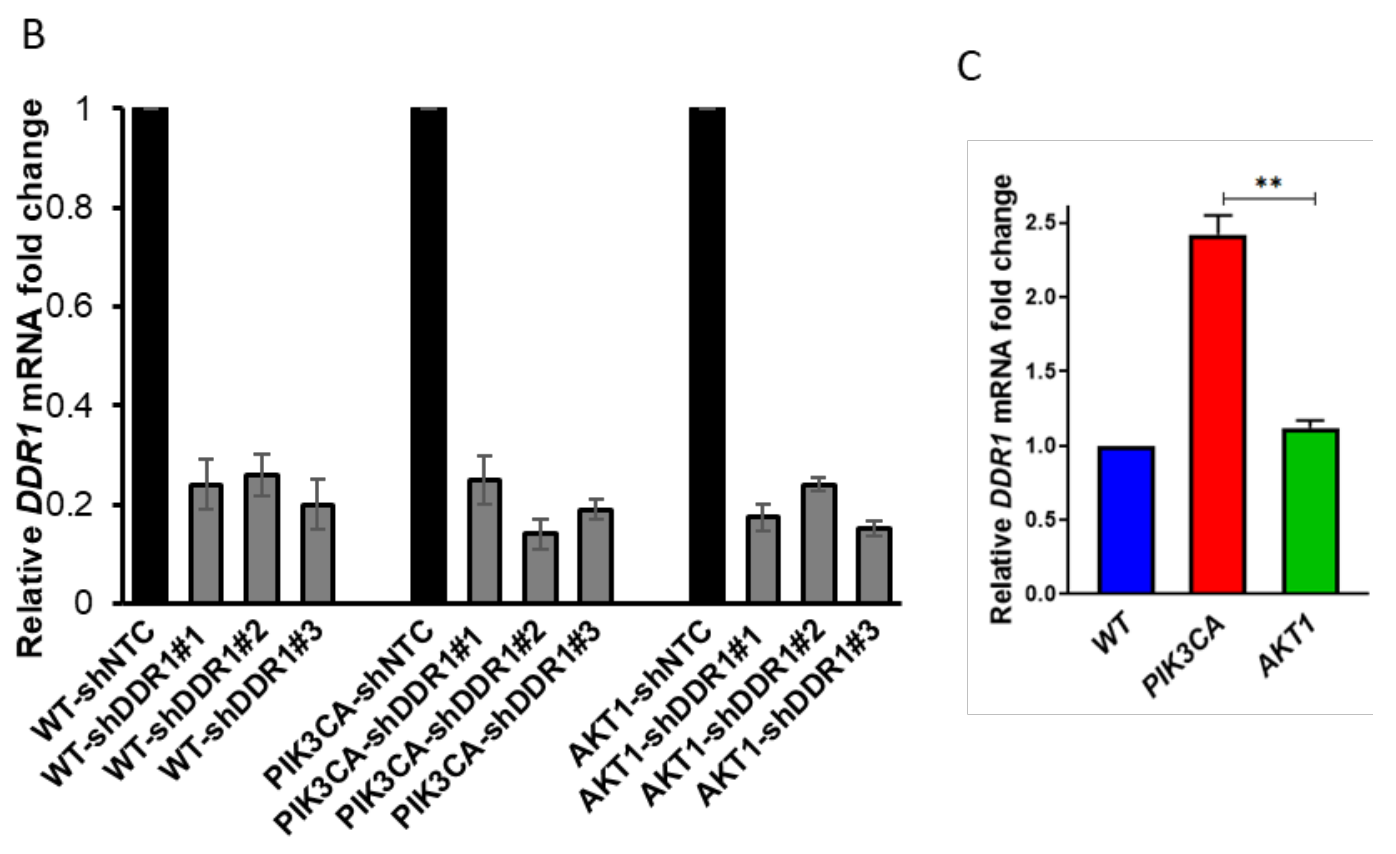

Figure 18. DDR1 protein and mRNA level in shRNA-induced DDR1 silencing cell lines.A) MCF7 cell lines were infected with non- targeting control shRNA (shNTC) and three different shRNA targeting DDR1. After puromycin selection for 72 hours, the DDR1 protein level was determined by western blotting. B) qRT-PCR results quantifying $D D R 1$ mRNA level in DDR1 knockdown relative to control (shNTC) in each cell lines. C) qRT-PCR analysis of DDR1 mRNA level was 
evaluated in PIK3CA and AKT1 mutant cells relative to WT cells. Normalization was done using human GAPDH as housekeeping control gene.

\subsubsection{DDR1 suppression inhibits proliferation and selectively sensitizes PIK3CA/AKT1 mutant cells to palbociclib treatment}

To verify the selective sensitivity of PIK3CAVAKT1 mutant cells on cell viability and proliferation in response to DDR1 suppression and palbociclib treatment, the DDR1 knockdown cells were treated with vehicle or palbociclib for 3 weeks. We observed a remarkable decrease in colony formation in all shRNA-mediated DDR1 silencing cells, regardless of their genomic backgrounds, indicating that DDR1 plays a crucial role in cell survival. The addition of palbociclib induced a significant growth reduction in DDR1 knockdown PIK3CA and AKT1 mutant cells compared with control, whereas DDR1 knockdown WT cells exhibited a growth inhibition rate similar to control (Figures 19A and B).

We had shown earlier that PIK3CA and AKT1 mutations decreased palbociclib sensitivity; therefore, we examined whether DDR1 silencing enhances their response. Cell cycle analysis by flow cytometry confirmed that, in PIK3CAIAKT1 mutant cells, DDR1 knockdown combined with palbociclib induced a greater decrease in G2-M and S phases, whereas no significant change was observed in the shRNA control cells treated with palbociclib. There was no difference in palbociclib-induced cell cycle inhibition between DDR1 knockdown and 
control shRNA in WT cells (Figures $19 \mathrm{C}$ and $\mathrm{D}$ ). This indicates that DDR1 silencing sensitizes PIK3CAIAKT1 mutant cells to palbociclib through cell cycle suppression.

A
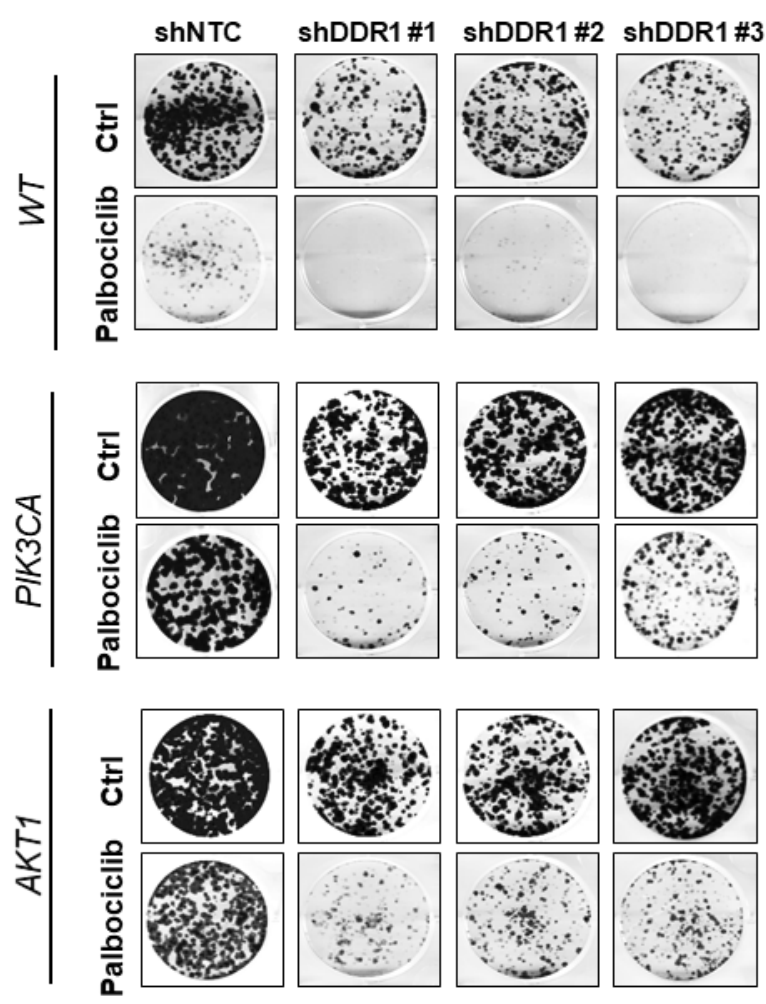


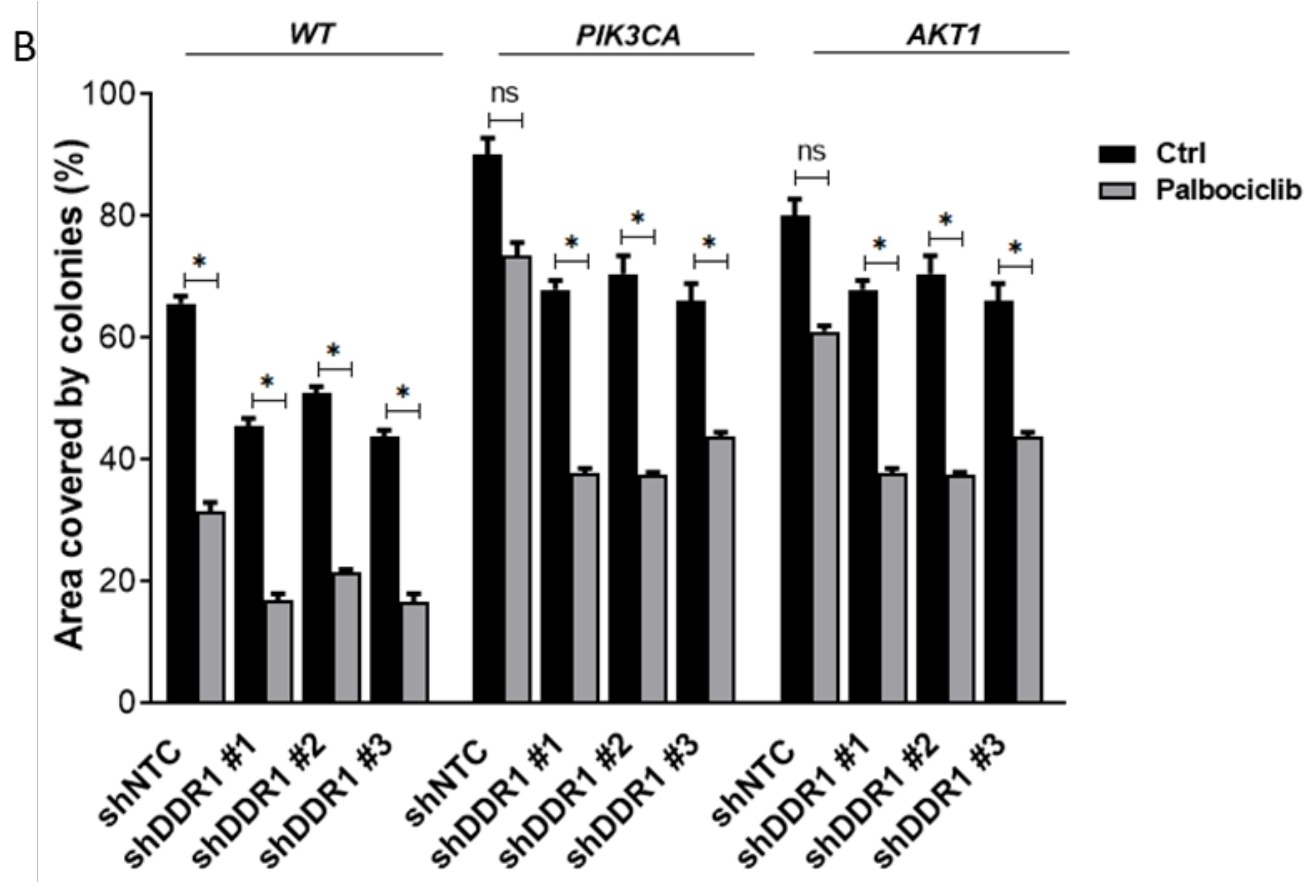



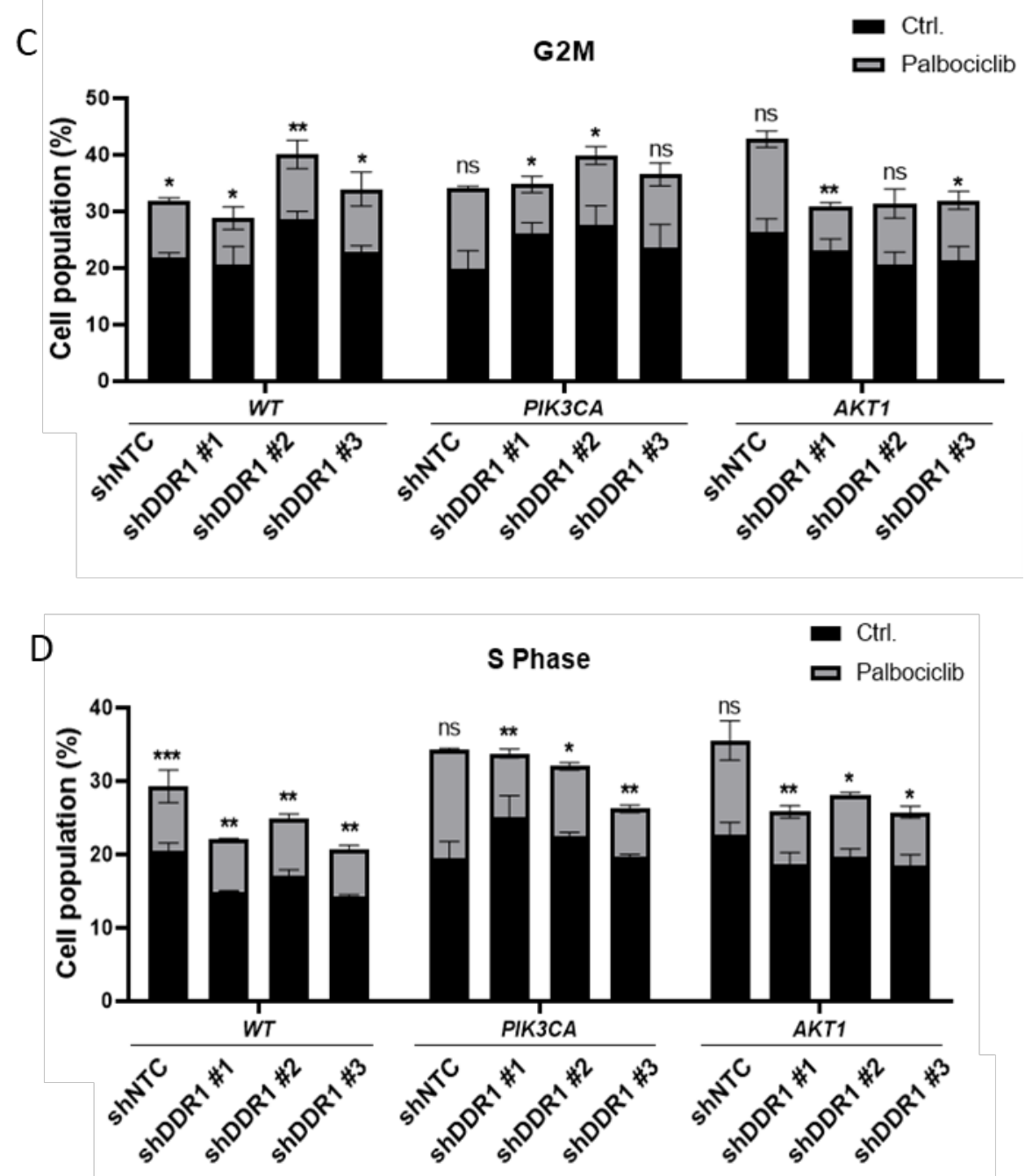

Figure 19. Silencing DDR1 sensitizes PIK3CA and $A K T 1$ mutant cells to palbociclib treatment. A) DDR1 knockdown cells were treated with $50 \mathrm{nM}$ of palbociclib and vehicle for three weeks. The colonies were then fixed and stained with crystal violet. B) Quantification of crystal violet staining from cells in A using $\mathrm{NIH}$ ImageJ v.1.46 software. C-D) DDR1 knockdown cells were treated with $100 \mathrm{nM}$ of palbociclib for 24 hours. The cells were then fixed and stained with propidium iodide and the cell abundance in different phases of cell cycle were analyzed with flow cytometry. Bar graphs show the quantification analysis for the significance of changes in G2M (C) and S (D) phases. All data mean \pm SD are indicated using triplicate samples of each representative experiment. P-value calculated based on ANOVA: $\left.0.1234(\mathrm{~ns}), 0.0332\left(^{(}\right), 0.0021{ }^{(*}\right), 0.0002\left(^{\star \star *}\right)$ and $<0.0001\left(^{\star \star \star *}\right)$. 


\subsection{SUMMARY}

shRNA screening analysis identified DDR1 as a novel synthetic lethal partner that differentially augmented palbociclib sensitivity in PIK3CA and AKT1 mutant cell lines. Notably, DDR1 expression level was higher in PIK3CAVAKT1 mutant cells, indicating the role of DDR1 in growth enhancement and increased proliferation observed in those cell lines. It also suggests a correlation between DDR1 expression level and reduced palbociclib sensitivity. Increased DDR1 expression may potentially benefit PIK3CA/AKT1 mutations to resist the anti-proliferation effect of palbociclib. To evaluate the effect of DDR1 suppression on colony formation and cell cycle progression, we silenced DDR1 using three different shRNAs All three DDR1targeting shRNAs induced greater than $70 \%$ reductions in both protein and mRNA level. DDR1 suppression inhibited colony proliferation of PIK3CA and AKT1 mutant and wild-type lines while selectively providing synergistic effect with palbociclib in mutant cell lines (Figures 19A and B) consistent with the previous survival assay results.

CDK4/6 form a complex with cyclin D1 to hyperphosphorylate and inactivate RB, allowing entry to S phase and cell cycle progression $[174,175]$. CDK4/6 inhibition blocks RB phosphorylation and subsequently leads to cell cycle arrest at G1, reducing the cell population at S phase [176]. Palbociclib treatment significantly inhibited the cell cycle at S phase in PIK3CA and AKT1 mutant cells expressing three different DDR1-targeting shRNAs but not in the cells with control shRNA 
(Figure 19D). Silencing DDR1 did not improve palbociclib-induced cell cycle arrest in WT cells. Hence, DDR1 suppression induces cell cycle inhibition effect of palbociclib reduced by PIK3CA and AKT1 mutations. These data further support the role of DDR1 in enhancing palbociclib sensitivity in ER-positive breast cancer with PIK3CA or AKT1 mutations. Investigating molecular signaling alterations in response to DDR1 inhibition will help elucidate the functional role of DDR1 as a novel target in breast cancer. 


\section{CHAPTER 6: DDR1 INHIBITION WITH 7RH SENSITIZES PIK3CA AND AKT1 MUTANT CELLS TO PALBOCICLIB TREATMENT BY INDUCING CELL CYCLE ARREST}

\subsection{INTRODUCTION}

Targeting $D D R 1$ by using selective available pharmacological agents is another approach to further validate the synergistic effect of DDR1 and CDK4/6 combined inhibition. Generally, there are two types of non-covalent kinase inhibitors. Type I competes with ATP for binding to the kinase active site (ATP-binding pocket) and maintains an active conformation, whereas type II occupies the allosteric site stabilizing an inactive kinase conformation $[177,178]$. Among the FDA-approved small-molecule protein kinase inhibitors, dasatinib, nilotinib, imatinib [179], and ponatinib [180] potently suppress DDR1 activity while strongly inhibiting a panel of other kinases (Table 6 and Table 7). These multi-targeted drugs were rationally developed for targeted therapy of chronic myeloid leukemia (CML) with Bcr-Abl tyrosine kinase alterations.

Given the emerging role of DDR1 as a potential anti-cancer target, several compounds have been discovered to selectively inhibit DDR1 kinase activity, with less efficacy against other kinases (Table 7) [181-183]. A series of benzamide compounds has been reported to have encouraging selective DDR1 inhibition effects. The two most promising benzamide compounds, 7rh and 7rj, were shown to 
inhibit the kinase function of DDR1, with IC50 values of $6.8 \mathrm{nM}$ for $7 \mathrm{rh}$ and $7 \mathrm{nM}$ for 7rj [181]. They displayed significantly less efficacy against kinases such as DDR2 and Bcr-Abl. Moreover, 7rh and 7rj exhibited a good in vivo pharmacokinetic profiles with half-lives of 15.5 hours and 9.8 hours, respectively.

\begin{tabular}{lllll} 
Imatinib & Nilotinib & Dasatinib & Ponatinib \\
\hline \hline ABL & ABL & ABL & BTK & ABL \\
ARG & ARG & ARG & TEC & BCR-ABL \\
BCR-ABL & BCR-ABL & BCR-ABL & BMX & c-KIT \\
c-KIT & C-KIT & C-KIT & TXK & FLT3 \\
PDGFR & PDGFR & PDGFR & DDR1 & FGFR \\
DDR1 & DDR1 & SRC & DDR2 & RET \\
NQO2 & NQO2 & YES & ACK & PDGFR \\
DDR2 & DDR2 & FYN & BRAF & VEGFR \\
& & LYN & EGFR & SRC \\
& & HCK & EPHA & DDR1 \\
& & LCK & MAPK & DDR2 \\
& & FGR & RAF & \\
& & BLK & SLK & \\
& & FRK & ZAK & \\
\hline \hline
\end{tabular}

Table 6. FDA-approved non-selective oral DDR1 and DDR2 inhibitors. The kinases targeted by each inhibitor are listed in the columns.

The PISK/AKT pathway positively regulates survival by activating the proteins with critical roles in cell cycle progression, such as cyclin D1 [139]. Therefore, DDR1 inhibition may differentially impact the protein expression of known palbociclib effectors in PIK3CA and AKT1 mutant cells. We used 7rh (DDR1 selective pharmacological inhibitor) in combination with palbociclib to further validate the synergistic effect of DDR1 and CDK4/6 combined inhibition on colony-forming ability and cell cycle progression. To determine the molecular signaling responsible for 
improving palbociclib response in the presence of DDR1 inhibitor, we examined the protein levels of known palbociclib effectors as well as other potential proteins involved in cell signaling.

\begin{tabular}{lccl} 
Compounds & DDR1 IC50 (nM) & DDR2 IC50 (nM) & Inhibitor type \\
\hline Dasatinib & $0.5 \pm 0.2$ & $1.4 \pm 0.3$ & Type I \\
Nilotinib & $43 \pm 3$ & $55 \pm 9$ & Type II \\
Imatinib & $337 \pm 56$ & $675 \pm 127$ & Type II \\
Ponatinib & 9 & 9 & Type II \\
7rh & 6.8 & 101.4 & Type II (benzamides) \\
7rj & 7 & 93.6 & Type II (benzamides) \\
DDR1-IN-1 & 105 & 413 & Type II \\
DDR1-IN-2 & 47 & 145 & Type II \\
2a & 68 & 65 & Type II \\
4a & 235 & 75 & Type II \\
4b & 39 & 18 & Type II
\end{tabular}

Table 7. Reported selective and non-selective DDR1 and DDR2 inhibitors. The half-maximal inhibitory concentration (IC50) values against DDR1 and DDR2 and the kinase inhibitor type for each compound are indicated.

\subsection{RESULTS}

\subsubsection{DDR1 and CDK4/6 co-inhibition induces a synergistic response and anti-proliferation effect in PIK3CA and $A K T 1$ mutant cells}

To develop a therapeutic strategy that would enhance palbociclib sensitivity reduced by PIK3CA and AKT1 mutations, we used 7rh benzamide (Figure 20A), an orally available ATP-competitive selective DDR1 inhibitor with potent DDR1 
inhibition in preclinical models [184]. Cells were treated with varying concentrations of 7rh $(0.01-100 \mu \mathrm{M})$ and palbociclib $(0.001-10 \mu \mathrm{M})$ as single and combined agents for 72 hours. All cell lines showed decreased survival to 7rh in a dose-dependent manner, and the addition of palbociclib induced synergy with stronger effect in PIK3CA and AKT1 mutant cells with consistent CI values <1(Figure 20B). We performed clonogenic assay to further assess growth inhibition in response to 7rh, palbociclib and their combination at various doses. Compared with control, MCF7WT exhibited a robust growth inhibition in all treatment groups. However, combinations at various doses demonstrated a significant growth reduction compared with single-agent treatment in PIK3CA and AKT1 mutant cells (Figures 20C-E).

A<smiles>CCc1ccc(C(=O)Nc2cc(CN3CCN(C)CC3)cc(C(F)(F)F)c2)cc1C#Cc1cnc2ccnn2c1</smiles> 

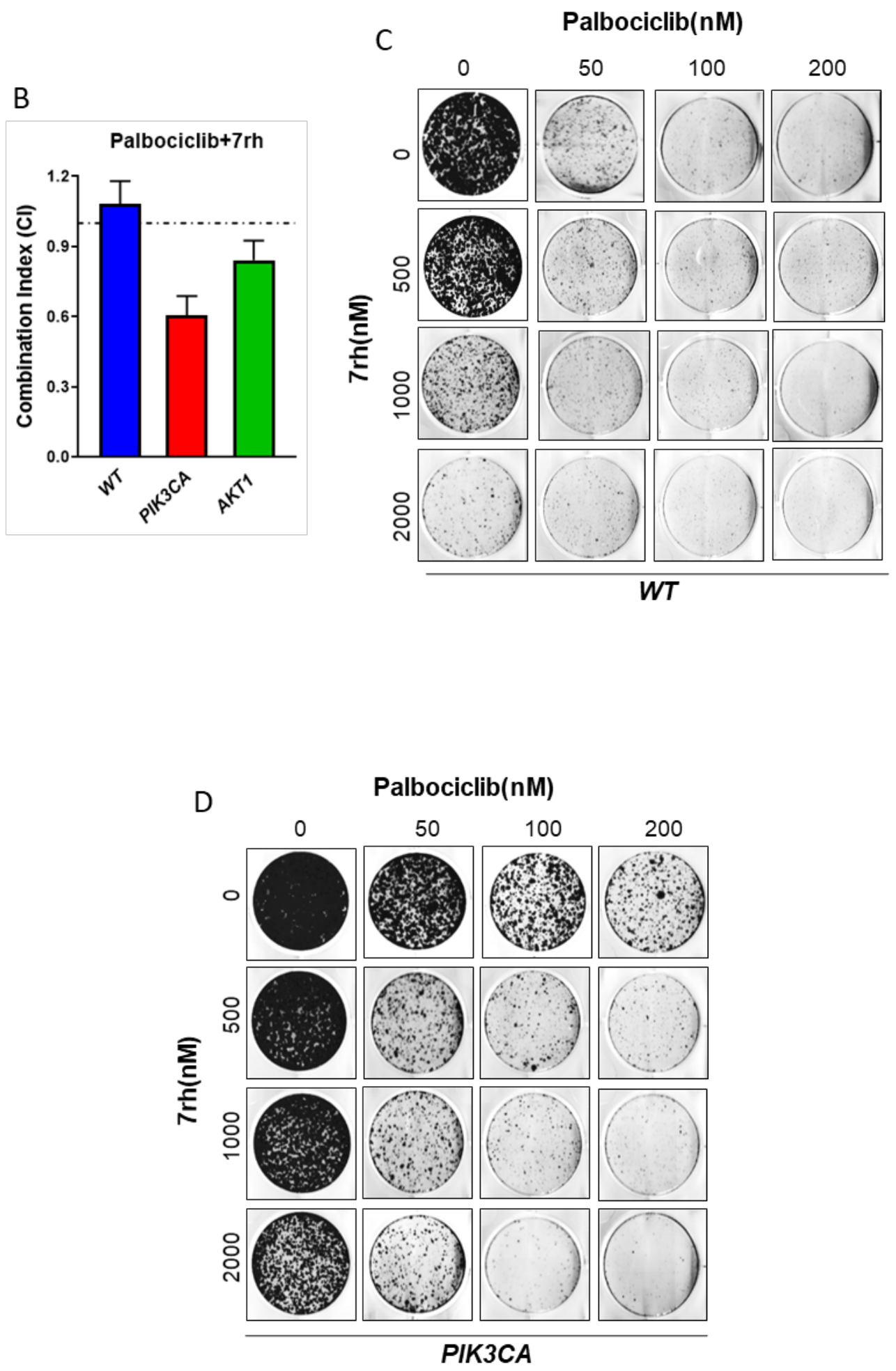


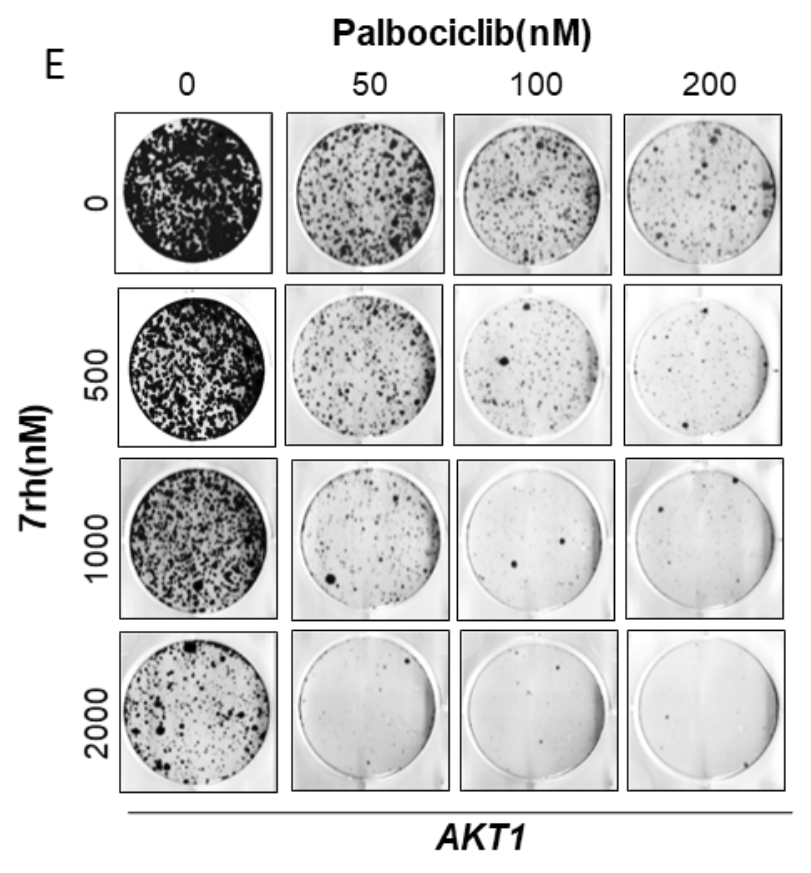

Figure 20. DDR1 and CDK4/6 co-inhibition induces more sensitivity and antiproliferation effect in PIK3CA and AKT1 mutant cells. A) Structure of 7rh benzamide compound: (3-(2-(pyrazolo(1,5-a)pyrimidin-6-yl)-ethynyl). B) MCF7 isogenic cell lines were treated with five different concentrations of 7rh, palbociclib, the combination of $7 \mathrm{rh}$ and palbociclib or vehicle in a ten-fold serial dilution series for 72 hours. SRB assay was used to assess cell growth effect and the $\mathrm{Cl}$ values were calculated using dose-effect analyzer software CalcuSyn 2.0. C-E) Cells were treated with increasing concentrations of 7rh, palbociclib or the combination of 7rh and palbociclib for three weeks. The colonies were fixed and stained with crystal violet then counted using NIH ImageJ v.1.46 software.

\subsubsection{DDR1 inhibition enhances palbociclib-induced cell cycle arrest of}

\section{PIK3CA and AKT1 mutant cells}

We next examined the effect of palbociclib and 7rh treatment on cell cycle progression. The cells were treated for 24 hours with $7 \mathrm{rh}$ or palbociclib alone or with 
a combination of both compounds, then harvested and subjected to flow cytometric analysis. As expected, palbociclib dramatically decreased the population of WT cells in S and G2-M phases, whereas the reduction was modest in PIK3CA and AKT1 mutant cells. The addition of 7rh to palbociclib produced smaller percentages of PIK3CA and AKT1 mutant cells in S and G2-M phases as compared with single agents, suggesting cell cycle arrest. However, WT cell percentages remained unchanged (Figures 21A-B). To further explore the enhanced effect of DDR1 inhibitor on palbociclib induced cell cycle inhibition, cells were treated with 7rh, palbociclib, or the combination of both drugs for 24 hours.

Immunoblot analysis of known palbociclib effectors, pRB and FoxM1, [185] in addition to other cell cycle regulators was performed. The results showed that both 7rh and palbociclib individually caused differential decreases in RB phosphorylation, FoxM1, and PLK1 levels in all cell lines, but the combination yielded a greater reduction in only PIK3CA and $A K T 1$ mutant cells (Figure $21 \mathrm{C}$ ). Consistent with the work of others [186, 187], the level of c-Myc, a critical positive cell cycle regulator, decreased in all treatment groups while cyclin D1 increased. These results suggest that the presence of PIK3CA and AKT1 mutations prevent sufficient reduction in RB phosphorylation in response to palbociclib, and 7rh can further suppress the residual phosphorylation in the context of co-treatment. Therefore, full suppression of cell cycle by palbociclib in PIK3CA and AKT1 mutant cells is facilitated through DDR1 inhibition. 
A

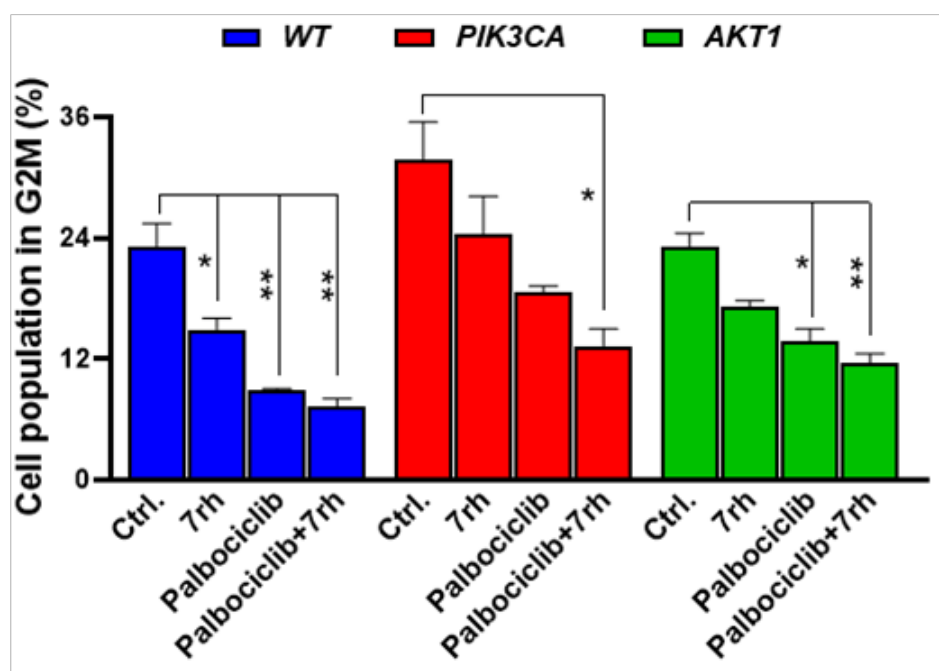

B

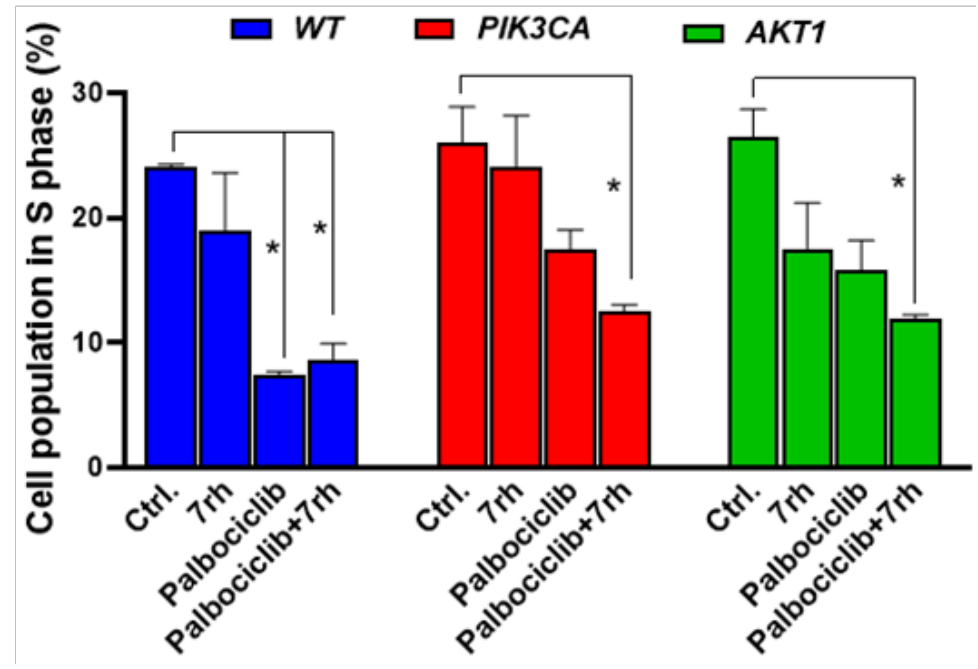




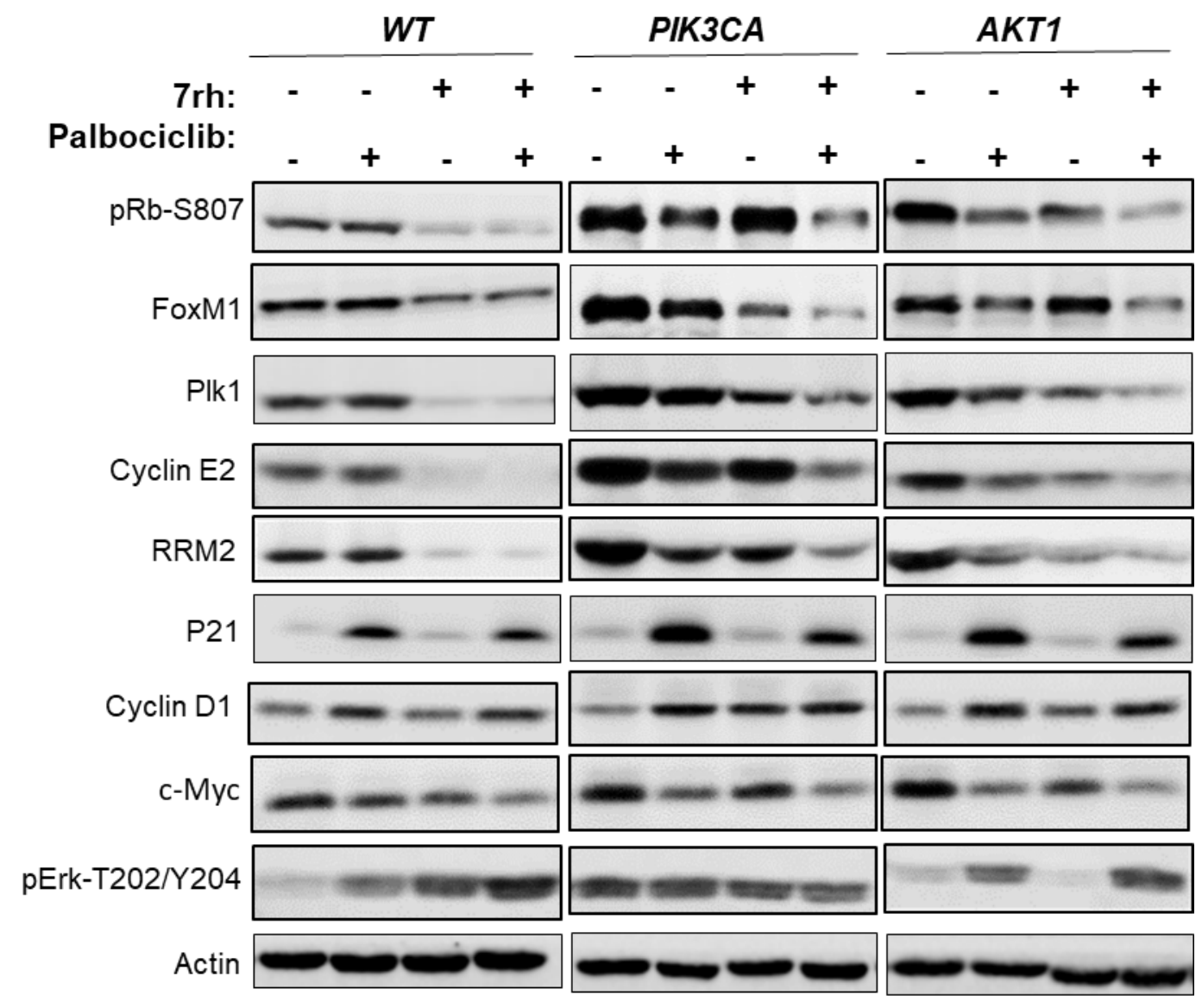

Figure 21. DDR1 inhibition enhances palbociclib-induced cell cycle arrest of PIK3CA and AKT1 mutant cells. A-B) Cell were treated with $100 \mathrm{nM}$ of palbociclib, $1 \mu \mathrm{M}$ of $7 \mathrm{rh}$ or the combination of both drugs for 24 hours. The cells were then fixed and stained with propidium iodide and the cell abundance in different phases of cell cycle were analyzed using flow cytometry. Bar graphs show the quantification analysis for the significance of changes in G2-M (A) and S (B) phases. C) MCF7 isogenic cell lines were treated with $1 \mu \mathrm{M}$ of $7 \mathrm{rh}, 100 \mathrm{nM}$ of palbociclib or the combination of $7 \mathrm{rh}$ and palbociclib for 48 hours. The expression levels of cell cycleassociated proteins were analyzed by western blotting. All data mean \pm SD are indicated using triplicate samples of each representative experiment. P-value calculated based on ANOVA: $0.1234(\mathrm{~ns}), 0.0332\left(^{\star}\right), 0.0021\left(^{(*}\right), 0.0002\left(^{\star \star *}\right)$ and $<$ $0.0001(* \star \star *)$. 


\subsubsection{DDR1 inhibition enhances palbociclib-induced cell cycle arrest of ER- positive breast cancer cell lines with PIK3CA mutations}

To further evaluate the synergistic effect of DDR 1 and CDK4/6 co-inhibition, we examined KPL-1 (PIK3CA-E545K mutation), BT483 (PIK3CA-E542K mutation), and T47D (PIK3CA-H1047R mutation) ER-positive cell lines. Likewise, SRB cell proliferation and colony formation assays revealed that $7 \mathrm{rh}$ potently inhibited the growth of other ER-positive, HER2-negative luminal breast cancer cells with PIK3CA mutations (KPL-1, BT483, and T47D) in a dose-dependent manner and suppressed anchorage-dependent growth. The combination of palbociclib with 7rh to induce DDR1 inhibition further decreased colony-forming ability and induced palbociclib sensitivity in KPL-1 and BT483 cells but not in T47D cells, as shown by clonogenic and SRB assays (Figures 22A and B).

To investigate the combined effect of $7 \mathrm{rh}$ and palbociclib on cell cycle signaling in KPL-1 and BT483 cell lines, the relevant protein expression levels were evaluated by using western blotting. Notably, dual treatment with 7rh and palbociclib markedly decreased the protein expression levels of pRB, PLK1, and RRM2, compared with 7rh or palbociclib alone (Figure 22C). These results indicated that the cell cycle signaling pathway was significantly suppressed after treatment with 7rh and palbociclib in PIK3CA mutant ER-positive breast cancer cells. 

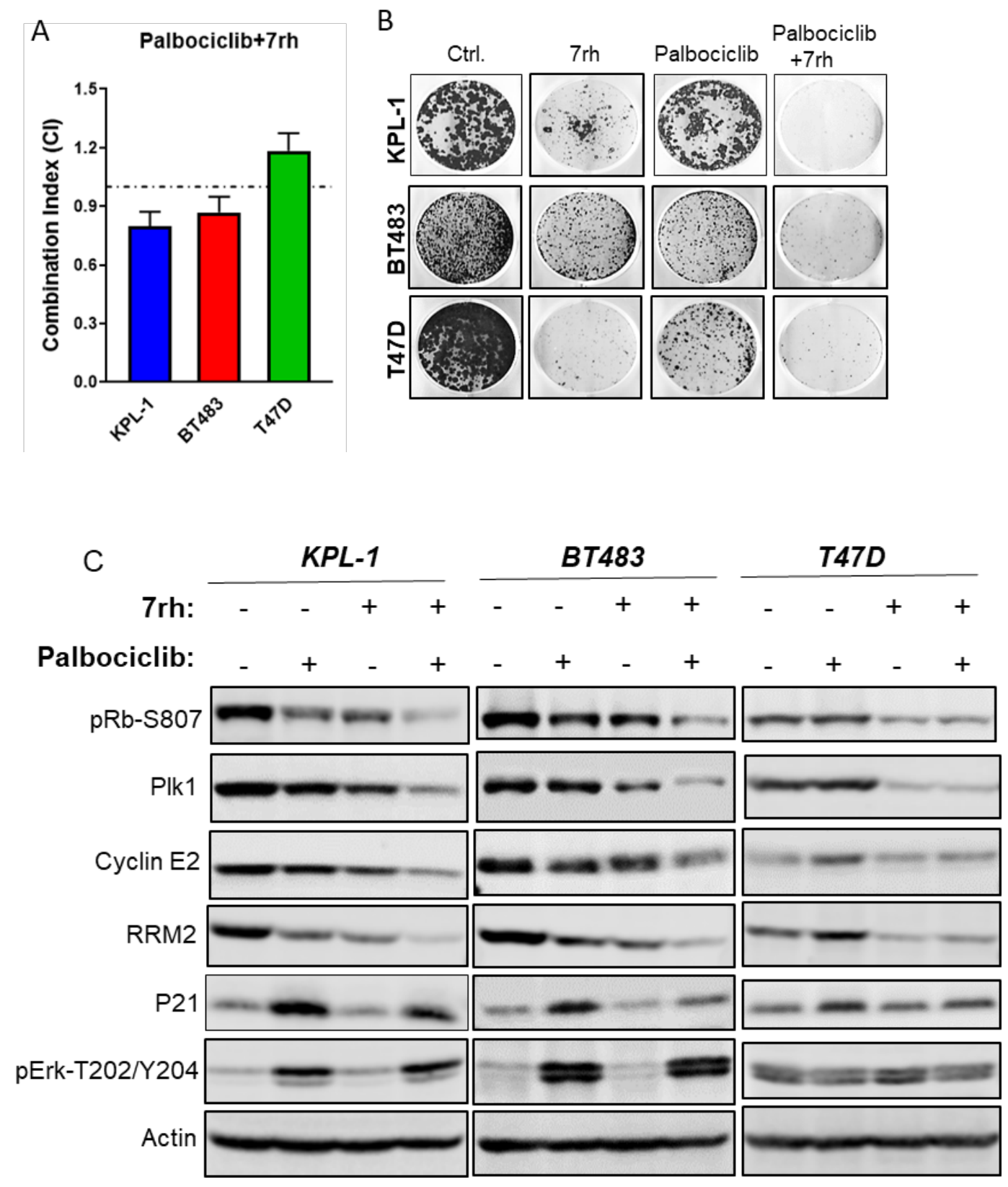

Figure 22. The effect of DDR1 and CDK4/6 co-inhibition in ER-positive PIK3CAmutated breast cancer cell lines. A) KPL-1, BT483, and T47D cell lines were treated with five different concentrations of 7rh, palbociclib, the combination of 7rh and palbociclib and vehicle in a ten-fold serial dilution series for 72 hours. SRB assay was used to assess cell growth effect and the $\mathrm{Cl}$ values were calculated using dose-effect analyzer software CalcuSyn 2.0. B) Cells were treated with 7rh, palbociclib or the combination of 7rh and palbociclib for three weeks. The colonies were fixed and stained with crystal violet then counted using NIH ImageJ v.1.46 software. C) KPL-1, BT483, and T47D cell lines were treated with $1 \mu \mathrm{M}$ of 7rh, 100 
$\mathrm{nM}$ of palbociclib or the combination of 7rh and palbociclib for 48 hours. The expression levels of cell cycle-associated proteins were analyzed by western blotting.

\subsection{SUMMARY}

Treatment with 7rh or palbociclib as a single agent decreased RB phosphorylation and, FoxM1 and PLK1 levels in all ER-positive breast cancer cell lines; combination treatment reduced PIK3CA and AKT1 mutant cells in all but the T47D cell lines. A study has shown that exon 9 PIK3CA mutations (E545K and E542K) in the helical domain are affiliated with AKT1 activation, which is essential for E2-dependent ERa signaling, whereas exon 20 PIK3CA mutation (H1047R) in the kinase domain impacts both AKT1 and AKT2 activations [188]. Similarly, pathological features analysis of clinical data revealed that exon 9 PIK3CA mutations are common in invasive lobular carcinomas and are associated with early recurrence and poor prognosis; conversely, exon 20 PIK3CA mutations are seen in all histological types of breast tumors and are associated with optimal prognosis [189]. Unlike other cell lines, T47D cells have PIK3CA mutation in the kinase domain, which may affect their sensitivity to combined 7rh and palbociclib treatment.

Palbociclib is expected to induce cell cycle arrest in G1-S phase through the inhibition of CDK4/6, which allow progression to S phase. We showed that the addition of 7 rh to palbociclib resulted in a greater reduction of cell population in $\mathrm{S}$ phase and G2-M phase in PIK3CA and AKT1 mutant cells, compared with single agents. Interestingly, the protein levels of PLK1, a major regulator of G2-M 
transition, decreased significantly in response to combined treatment. Further, PIK3CA and AKT1 mutant cells had a modest PLK1 reduction in response to palbociclib, whereas a higher level of PLK1 was detected in those cell lines than in WT cells. In a recent clinical study of ER-positive metastatic breast cancer, PLK1 inhibition induced a dramatic tumor suppression in cyclin D1-driven patient-derived xenografts with acquired resistance to palbociclib [190]. On the basis of these observations, PLK1 may have a potential role in driving palbociclib resistance in ERpositive breast cancer with PIK3CA or AKT1 mutations, and DDR1 could represent a therapeutic target for enhancing palbociclib sensitivity. 


\section{CHAPTER 7: PHENOTYPIC CHANGES FOLLOWING ECTOPIC EXPRESSION OF WILD-TYPE AND MUTANT DDR1}

\subsection{INTRODUCTION}

Aberrant alteration of RTKs is a common indication in all human cancers and is implicated in disease pathogenesis and progression. Therefore, there is a great effort to identify and explore the functional impacts of genomic mutations for the development of optimal targeted therapies. DNA mutations as a result of genomic instability are categorized into driver or passenger mutations based on their contribution to cancer development. Driver mutations that are positively selected during tumorigenesis provide growth advantage and initiate cancer progression. Passenger mutations on the other hand do not confer cancer phenotype and are not essential for tumor survival. Sequencing approach to identify the mutational profiles in cancer to determine their relevant functional consequences is essential for effective targeted therapies. Determining whether the genomic mutations map to critical residues within the tyrosine kinase domain can predict their functional impact on kinase activity.

DDR1, a RTK with crucial roles in important cellular processes, has the unique ability to interact with ECM through collagens and to dysregulate the tumor microenvironment driving cancer invasion and aggressiveness [191]. Abundant expression of DDR1 mRNA and elevated protein levels were reported in invasive 
ductal carcinomas and lymph node metastases [192], whereas invasive lobular carcinomas and most of the intermediate- to high-grade breast carcinomas exhibited a significant reduction in DDR1 mRNA and protein levels [193, 194]. Several DDR1 somatic mutations mapped to various regions on the domain are reported in lung cancer [195] and acute myeloid leukemia (AML) [196]; however, identifying the potential involvement of these mutations in cancer progression requires further exploration. A few studies have identified DDR1 mutational status and examined its functional consequences in some cancer types [197], yet whether these alterations act as driver or passenger mutations remains unclear. In a recent clinical analysis, targeted sequencing of a large cohort of HR-positive breast cancer patients identified several DDR1 somatic mutations (Figure 23 ) that were strongly associated with poor outcomes ( $q$-value $=0.0003)$ [198]. Thus, to determine the transformational ability of the DDR1 mutations detected in HR-positive breast cancer patients (Figure 23), we overexpressed these mutations in MCF10A benign breast epithelial cell line. Several growth assays were performed to examine the proliferative effect of DDR1. 


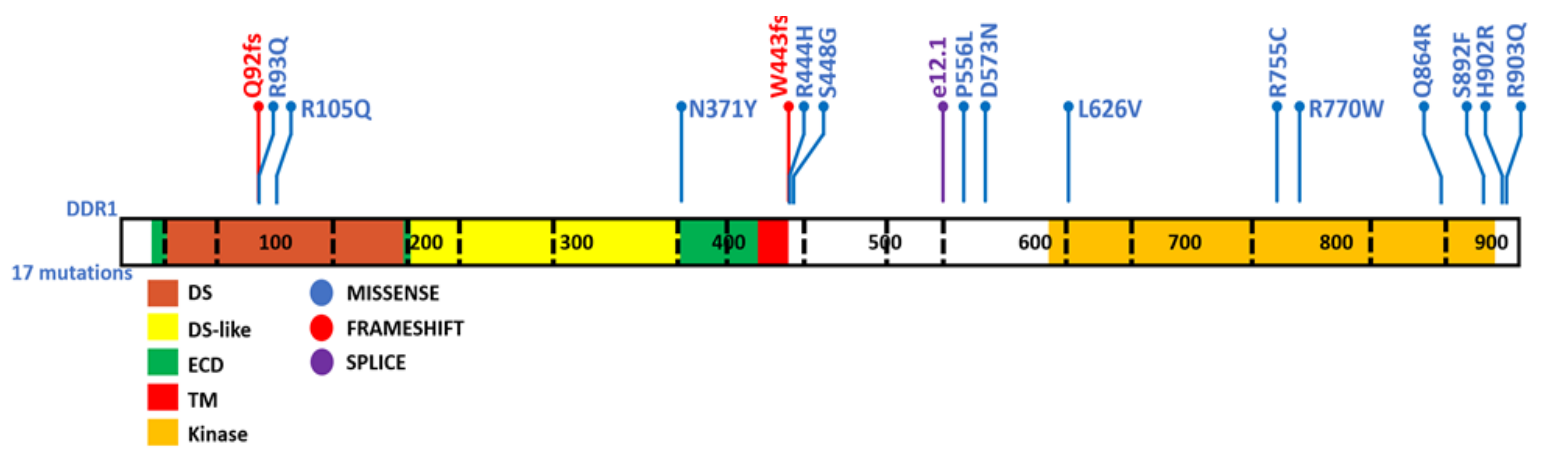

Figure 23. Mutation profile and frequency plot for $D D R 1$ transcript. The plot illustrates all non-silent mutations for DDR1. The DDR1 (RefSeq ID: NM_013994) domains are indicated below the schematic diagram. The ECD (extracellular domain), TM (transmembrane domain), and kinase domain are depicted as green, red, and orange bars respectively. Note, in the mapping from Ensembl to RefSeq annotation, the DDR1 variant annotation may have changed or been lost, despite selecting the most similar representative transcript possible. (Adopted with permission from: Griffith, O.L., et al., Author Correction: The prognostic effects of somatic mutations in ER-positive breast cancer. Nat Commun, 2018)

\subsection{RESULTS}

\subsubsection{Phenotypic changes following ectopic expression of DDR1 in MCF10A benign breast epithelial cells}

To determine the transformational ability of DDR1, the MCF10A benign breast epithelial cell line was selected to overexpress DDR1 and control vector. After DDR1 ectopic expression, MCF10A overexpressing DDR1 showed enhanced proliferation and significant increase in clonogenic growth, compared with the control cells (Figures 24A and B). Consistently, we also observed induction of anchorageindependent growth in MCF10A cells after DDR1 overexpression, as shown by soft- 
agar colony formation assay (Figure 24C). MCF10A control cells were not able to form a soft-agar colony. DDR1-overexpressing MCF10A cells exhibited an increase in RB phosphorylation and c-Myc levels in response to DDR1 overexpression (Figure 24D). These results suggest a potential role for DDR1 in growth enhancement through induction of cell cycle. To test whether DDR1 increases cell growth and contributes to cell cycle inhibition sensitivity, we treated MCF10A cells with 7rh and palbociclib after DDR1 overexpression by using SRB growth assay. Interestingly, MCF10A cells overexpressing DDR1 displayed more sensitivity to 7rh ( $p=0.005)$ but not to palbociclib $(p=0.038)$, compared with parental cells (Figure 24E), suggesting a role for DDR1 in growth enhancement and cell cycle progression.

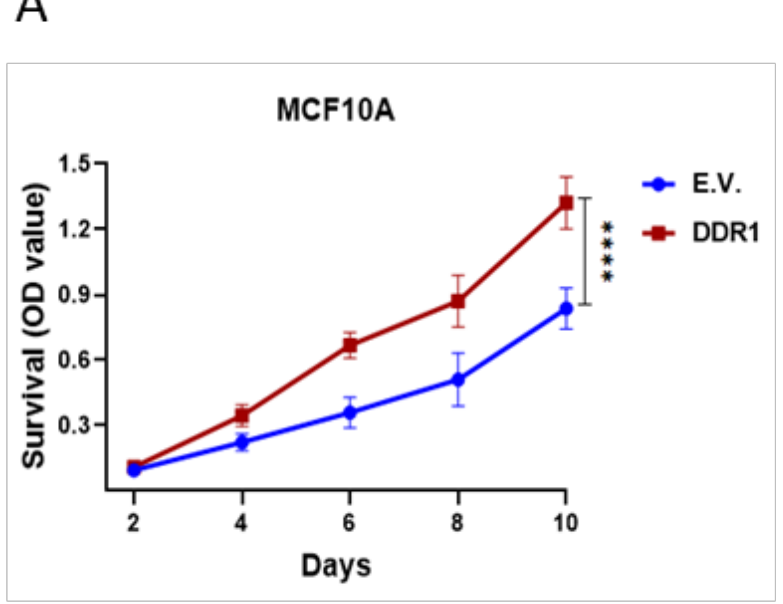

B

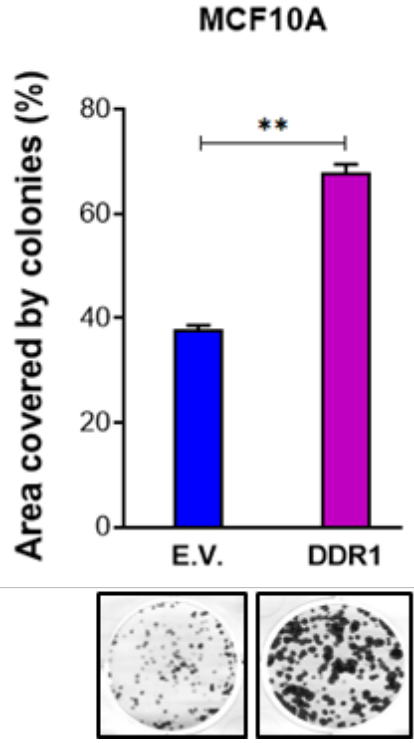


C

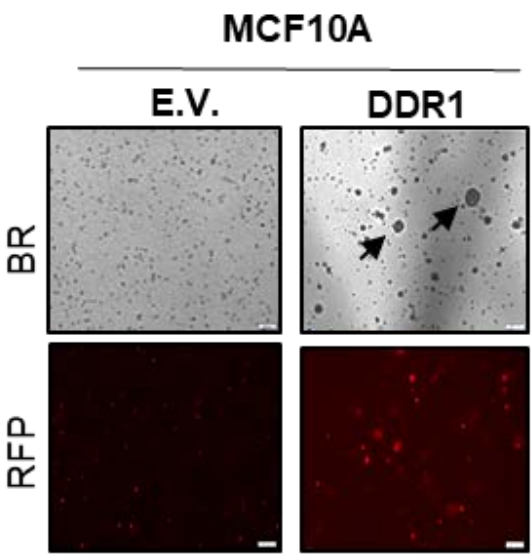

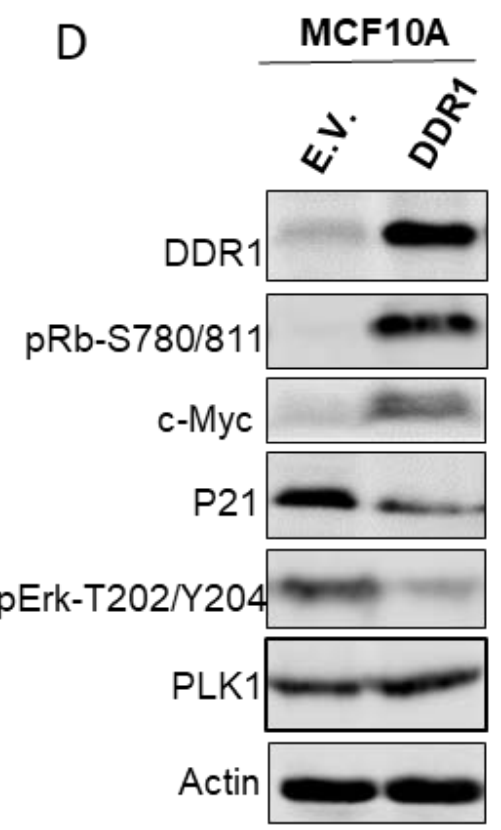

$\mathrm{E}$

\section{MCF10A}

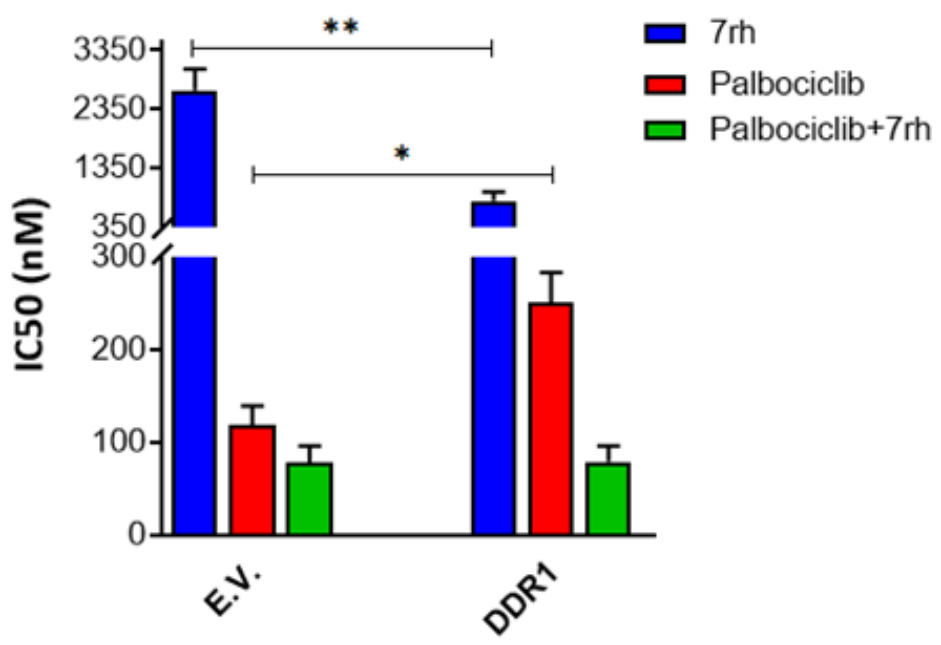

Figure 24. Phenotypic changes following ectopic expression of DDR1 in MCF10A benign breast epithelial cells. A) MCF10A cells were infected with empty vector (EV) and wild-type DDR1 constructs containing RFP tag. Following 48 hours puromycin selection, cells were seeded at 500 cells/well in 96 -well plates in triplicate. SRB assay was used to determine the survival of the cells every two days. B) The cells were seeded at 500 cells $/ 60 \mathrm{~mm}$ plates in triplicate. Colonies were then stained with crystal violet and counted using NIH ImageJ v.1.46 software after 3 weeks. C) Soft agar assay images of the MCF10A cells after four weeks of 
incubation. Colonies are indicated by the arrows. D) The expression levels of cell cycle-associated proteins were analyzed by western blotting after DDR1 overexpression in MCF10A cells. E) Cells were treated with five different concentrations of $7 \mathrm{rh}$, palbociclib or the combination of both drugs for 72 hours. SRB assay was used to assess cell growth effect and the IC50 values were calculated using dose-effect analyzer software CalcuSyn 2.0. All data mean \pm SD are indicated using triplicate samples of each representative experiment. P-value calculated based on ANOVA: 0.1234 (ns), $0.0332\left(^{(}\right), 0.0021\left(^{(\star}\right), 0.0002\left(^{\star \star \star}\right)$ and $<$ $0.0001\left(^{* \star * *}\right)$.

\subsubsection{Functional impact of reported DDR1 mutations in MCF10A benign breast epithelial cells}

$D D R 1$ amplification is commonly observed in various types of cancer, including metastatic breast cancer $(\sim 14 \%)[199,200]$. Recently, a large genomic analysis of ER-positive breast cancer patients identified uncommon mutations in DDR1 that were associated with early relapse and cancer recurrence [198]. To understand the differential effects of these mutations, we transduced MCF10A cells with lentivirus constructs expressing WT and the various DDR1 mutants. Compared with parental MCF10A cells (with empty vector), the MCF10A cells stably expressing wild-type $D D R 1$ or $D D R 1$ with mutations mostly in the kinase domain exhibited modestly higher levels of cell growth. However, in this screen, mutations did not significantly enhance growth beyond that seen with wild-type DDR1 overexpression (Figure 25A). Immunoblot analysis of MCF10A cells overexpressing wild-type DDR1 as well as several $D D R 1$ mutants revealed a differential increase in $\mathrm{RB}$ phosphorylation and c-Myc levels, suggesting activation of DDR1 signaling (Figure 25B). 


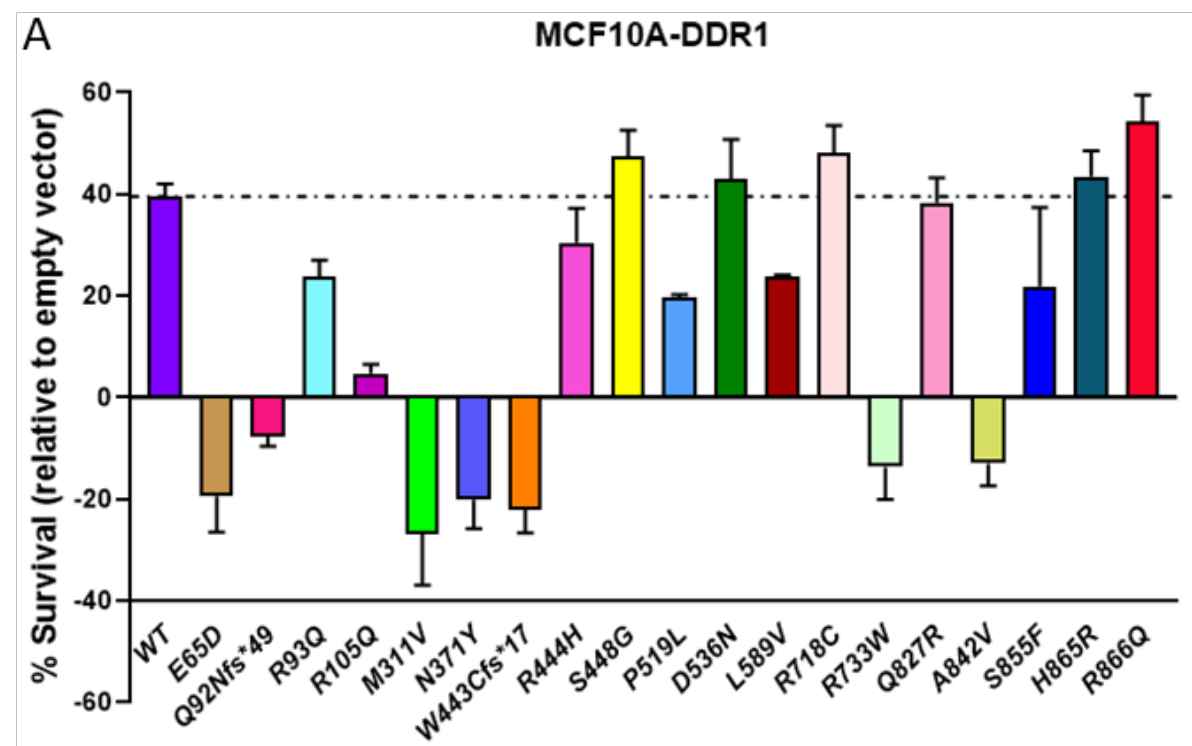

B
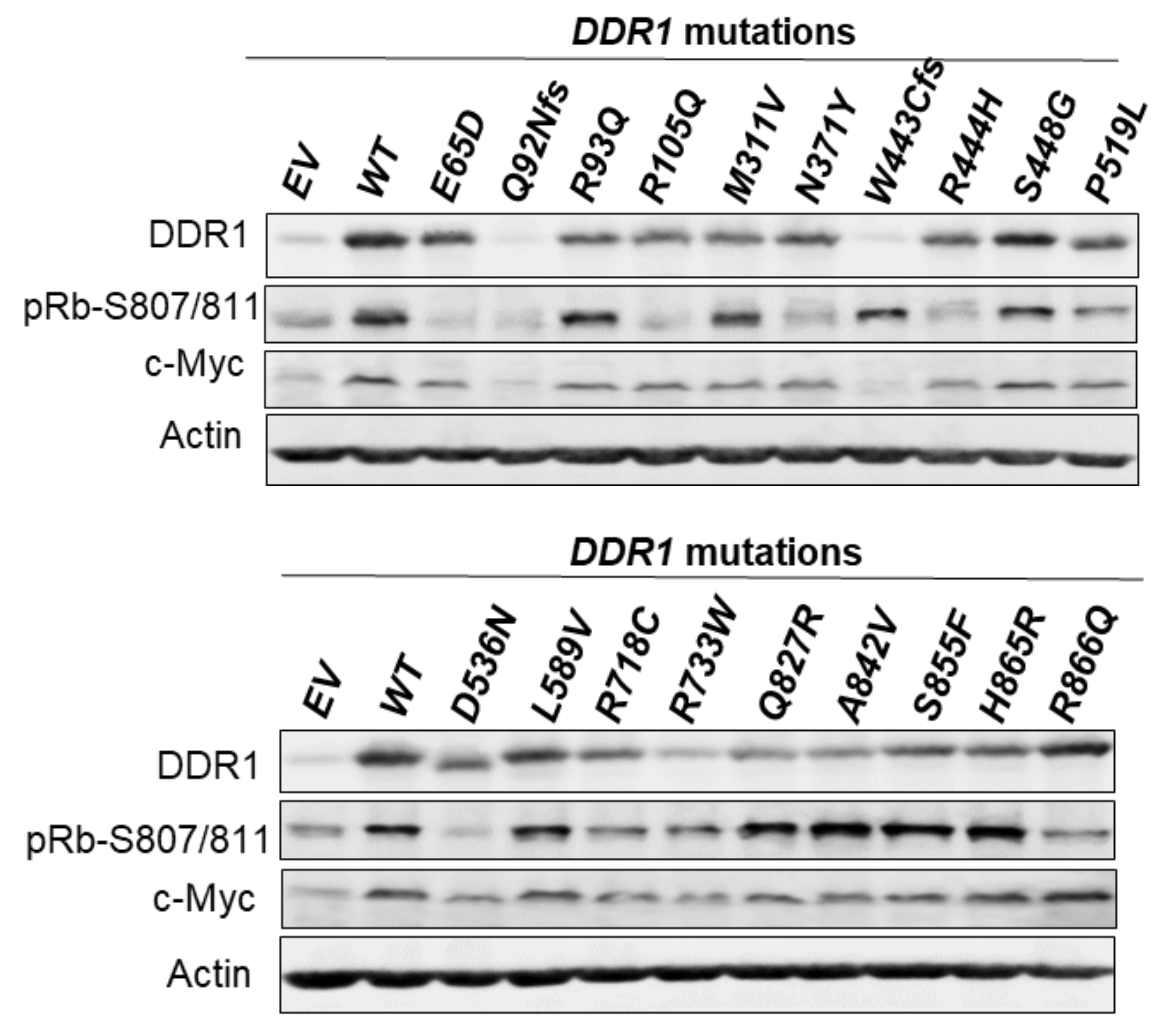
Figure 25. Functional impact of reported DDR1 mutations in MCF10A benign breast epithelial cells. A) MCF10A cells were transduced with lentivirus constructs expressing WT (wild-type) and various DDR1 mutations. Following puromycin selection, the survival was analyzed with SRB assay after 72 hours. B) Western blot validation of DDR1, RB phosphorylation (serine 807/811) and c-Myc in MCF10A cells expressing various $D D R 1$ mutations.

\subsubsection{Sensitivity to DDR1 inhibitor 7rh in a panel of breast cancer cell lines with different ER statuses and PI3K pathway mutations}

To assess the sensitivity of other breast cancer cell lines to 7rh, the IC50 in a panel of ER-positive and ER-negative cell lines with differential PI3K pathway mutations was determined (Figure 26). The results showed that ER-positive cell lines are more sensitive to 7rh than are ER-negative cell lines, perhaps due to higher expression of DDR1 as revealed in earlier TCGA and METABRIC dataset analyses (Figures 15A and B). 


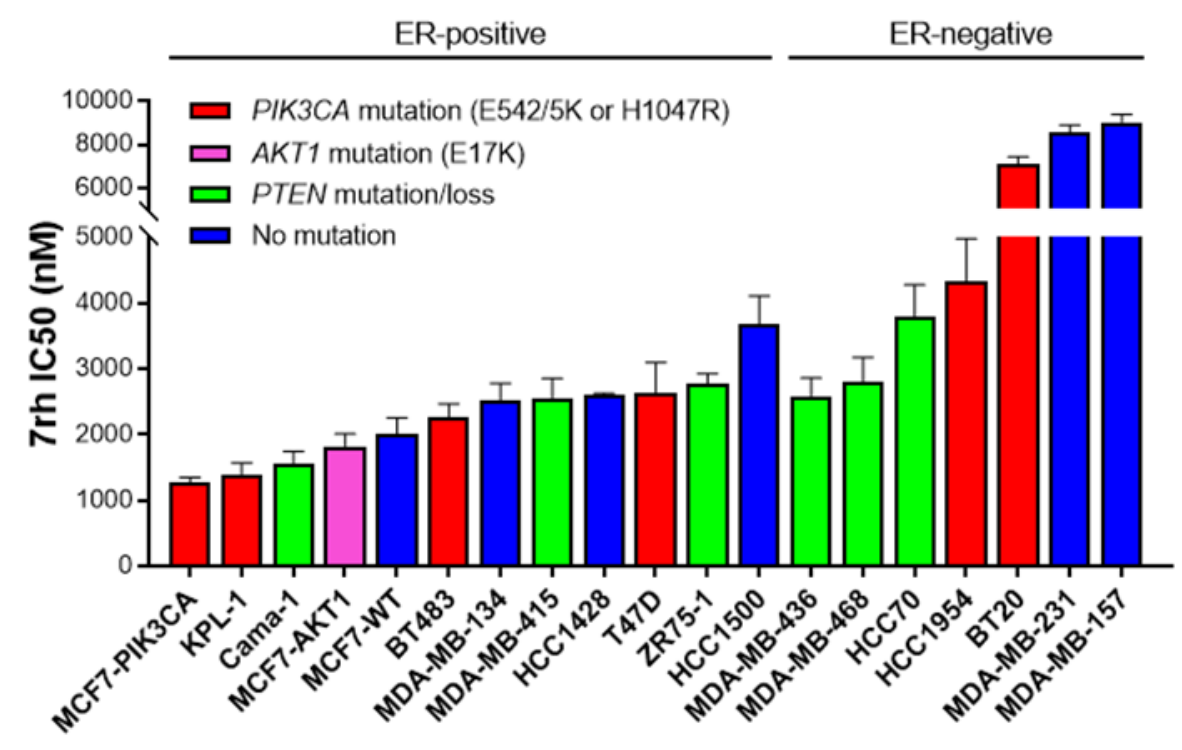

Figure 26. Sensitivity to DDR1 inhibitor 7rh in a panel of breast cancer cell lines with different ER statuses and PI3K pathway mutations. Bar chart showing 7rh sensitivity in a panel of ER-positive and ER-negative breast cancer cell lines with differential activation of PI3K pathway. All data mean \pm SD are indicated using triplicate samples of each representative experiment. P-value calculated based on ANOVA: $\left.0.1234(\mathrm{~ns}), 0.0332\left(^{(}\right), 0.0021{ }^{(* *}\right), 0.0002\left(^{(\star *}\right)$ and $<0.0001\left(^{\star * * *}\right)$. 


\subsection{SUMMARY}

Our data showed that the ectopic overexpression of DDR1 in MCF10A benign breast epithelial cells significantly increased cell growth and proliferation and induced anchorage-independent colony formation. Further, we demonstrated that MCF10A cells exhibited an increase in RB phosphorylation and C-Myc levels in response to DDR1 overexpression, suggesting a potential role for DDR1 in growth enhancement through induction of cell cycle. Interestingly, MCF10A cells overexpressing DDR1 were markedly more sensitivity to $7 \mathrm{rh}$ than were parental cells but were less responsive to palbociclib, indicating the involvement of DDR1 in attenuating palbociclib efficacy. The addition of 7rh inhibitor augmented palbociclib sensitivity in DDR1-overexpressing MCF10A cells, whereas no change was observed in parental cells. Taken together, these results support DDR1's prosurvival function and its role in alleviating palbociclib sensitivity.

MCF10A cells stably expressing DDR1 mutations detected in clinical samples exhibited distinct proliferation rates and various levels of phosphorylated RB and cMyc, compared with parental MCF10A (with empty vector). As anticipated, DDR1 protein level increased in all MCF10A cell lines harboring wild-type DDR1 as well as mutated DDR1, except the cells expressing Q92Nfs or W443Cfs frame-shift mutations. The c-Myc oncogene has been shown to collaborate with E2F in inducing the expression of target genes involved in cell cycle progression [201]. Notably, elevated DDR1 level was consistent with increased c-Myc expression across the MCF10A cell lines expressing DDR1 mutations, while RB phosphorylation level showed differential alterations regardless of DDR1 expression (Figure 25B). It is 
possible that DDR1 acts as an upstream mitogenic force to enhance c-Myc activation and promote cell cycle. In mammalian cells, c-Myc transcriptional activity can positively regulate cyclin $\mathrm{E}$, inducing G1-S transition in parallel with the $\mathrm{pRB} / \mathrm{E} 2 \mathrm{~F}$ pathway during cell cycle progression [202]. Nevertheless, elevated DDR1 and cMyc levels do not necessarily confer growth advantage. The expression of R93Q and M311V mutations induced and suppressed the proliferation of MCF10A cell lines respectively (Figure 25A), while both equally increased DDR1 and c-Myc protein levels (Figure 25B), compared with parental cells. The incomparable functional impacts of these mutations might be attributed to their distinct locations in the DDR1 domain. The R93Q mutation maps to ligand-binding domain (DS domain) responsible for receptor activation, whereas M311V is located on the less critical DS-like region of DDR1. After all, more investigations are required to determine the functional consequences of $D D R 1$ mutations in breast cancer progression.

The TCGA and METABRIC dataset analyses described above revealed that the ER-positive breast cancer subtype has a higher DDR1 expression (Figures 15A and B). Similarly, ER-positive cell lines displayed more sensitivity to 7rh-induced DDR1 inhibition (Figure 26). ER-positive cell lines without PI3K pathway alterations were more responsive to $7 \mathrm{rh}$ than were ER-negative cell lines having PIK3CA mutations. Meanwhile, PI3K pathway mutations provided more efficacy toward 7rhinduced DDR1 inhibition. These observations suggest a potential correlation between ER, DDR1, and PI3K pathway mutations that could enhance the targeted therapy of DDR1 tyrosine kinase in breast cancer. 


\section{CHAPTER 8: DDR1 INHIBITOR 7RH INHIBITES THE GROWTH OF PIK3CA XENOGRAFT TUMORS AND SYNERGIZES WITH PALBOCICLIB IN VIVO}

\subsection{INTRODUCTION}

As described earlier, endocrine therapy in combination with the selective CDK4/6 inhibitor palbociclib is the standard-of-care treatment for ER-positive, HER2negative metastatic breast cancer. Despite the initial efficacy of this therapy, patients eventually acquire resistance to palbociclib, thus attenuating its effectiveness. Considering the potential role of DDR1 inhibition in preventing palbociclib resistance induced by the frequently occurring PIK3CA mutations, it is crucial to explore the DDR1 molecular mechanism underlying cell cycle progression. Understanding the molecular and functional consequences of DDR1 activation will help in designing DDR1 targeted therapy to improve the efficacy of palbociclib in ER-positive, PIK3CA mutated advanced metastatic breast cancer.

To examine the synergistic effect of combined DDR1 and CDK4/6 inhibitions on improving palbociclib efficacy limited by PIK3CA mutation, we established MCF7PIK3CA xenograft tumors in NOD scid gamma (NSG) mice. This model has the advantage of mimicking tumor microenvironment and physiological conditions. RPPA analysis is a proteomic approach for molecular characterization of cultured cell lines and tissues. The use of RPPA can reveal variations in protein expression between samples in a short time. Exploring the expression of a large number of 
proteins in xenograft tumors treated with single-agent 7rh or palbociclib and their combination can help identify novel proteins critical for specific treatment response. The differential expressions of relevant as well as novel proteins in different treatment groups will help explain the molecular signaling responsible for the synergistic effect in the dual treatment of $7 \mathrm{rh}$ and palbociclib.

\subsection{RESULTS}

\subsubsection{DDR1 inhibitor 7rh inhibits the growth of PIK3CA xenograft tumors and synergizes with palbociclib in vivo}

To validate the synthetic lethality induced by 7rh and palbociclib in vivo, MCF7-PIK3CA xenograft tumors were established in NSG mice supplemented with $17 \beta$-estradiol pellets. Treatment with single-agent 7 rh or palbociclib inhibited tumor growth by $31 \%$ and $38 \%$, respectively (7rh vs. vehicle, $P=0.013$; palbociclib vs. vehicle, $P=0.0001$ ). Combination treatment significantly inhibited tumor growth by 49\% compared with vehicle $(P<0.0001)$ and each drug alone (combination vs. 7rh, $P=0.0001$; combination vs. palbociclib, $P<0.0004$ ) (Figure 27A). These results indicate that the combination of palbociclib and 7rh improved antitumor efficacy in vivo. The mice that received $7 \mathrm{rh}$ plus palbociclib exhibited no significant change in body weight, suggesting that this combination is well-tolerated.

Immunohistochemical staining revealed a lower level of proliferation marker Ki67 in palbociclib-treated tumors compared with controls $(P=0.0001)$, and the addition of

7rh further decreased the proliferation rate $(P<0.0001)$ (Figures 27B and $C)$. Tumors treated with either 7rh alone or palbociclib plus 7rh expressed a higher level of P21 
than did controls or tumors treated with palbociclib alone (Figure 27D). 7rh significantly increased P21 expression H-scores in tumors as a single treatment $(P=0.048)$ and in combination with palbociclib $(P=0.026)$ (Figure 27E).
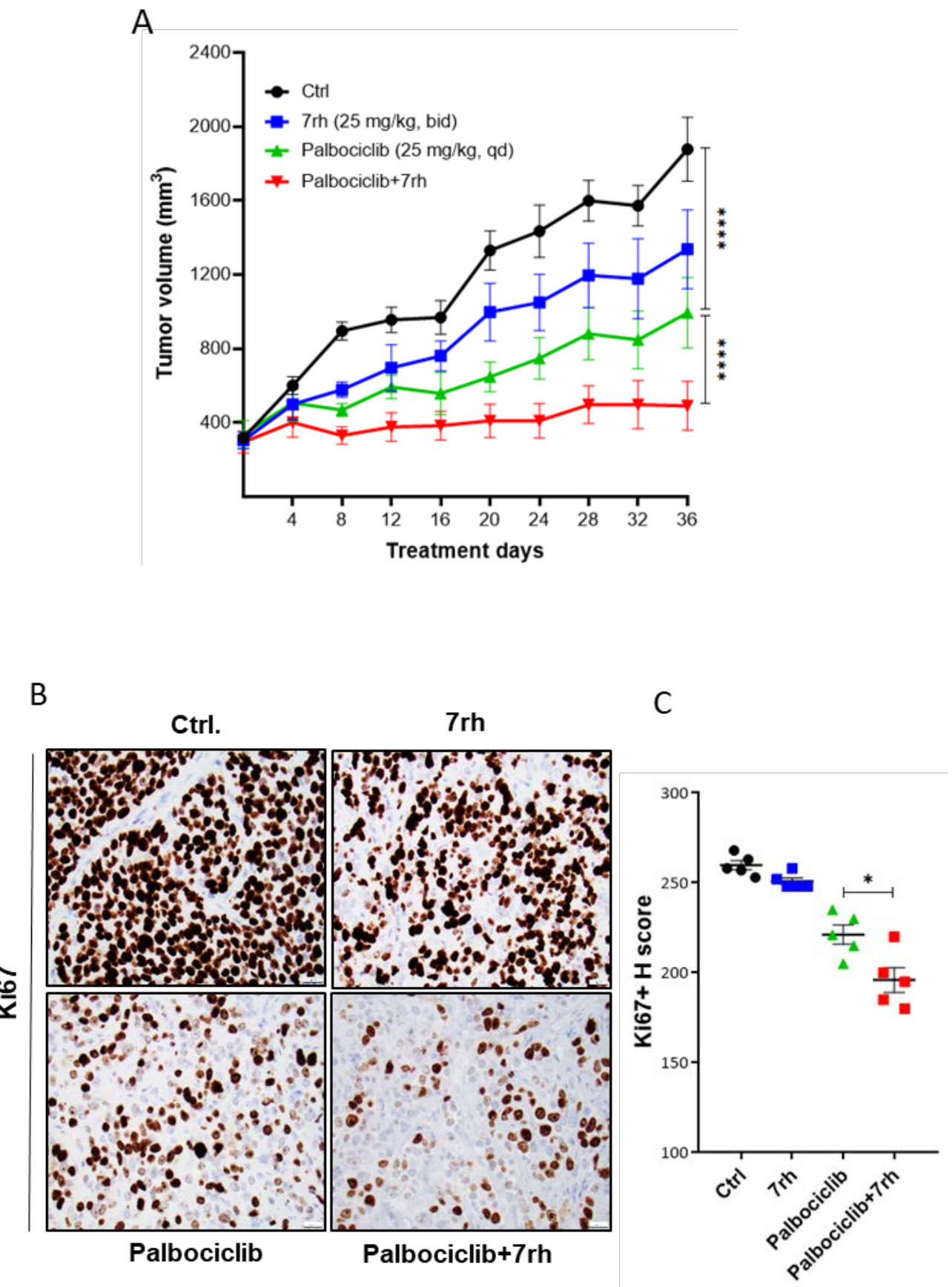
D

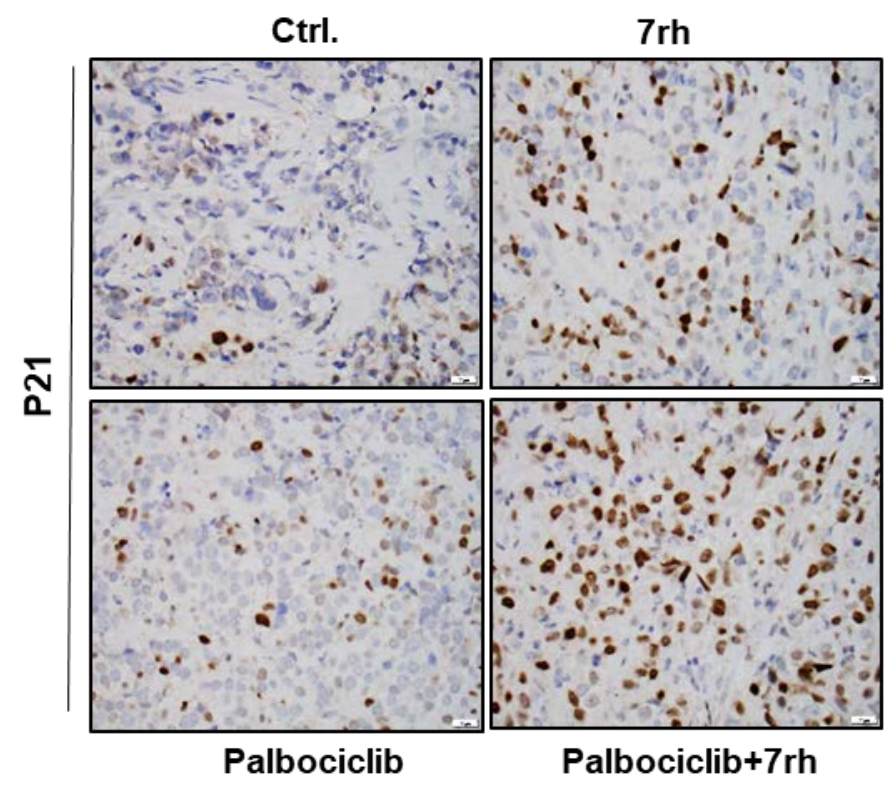

$E$

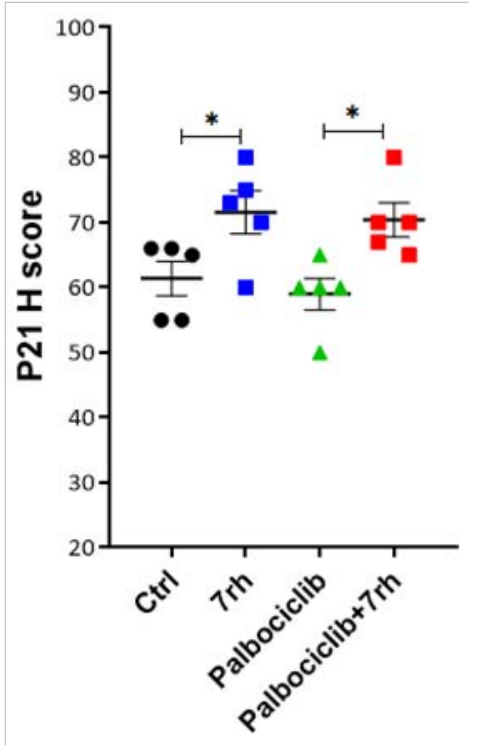

Figure 27. Dual inhibition of DDR1 and CDK4/6 decreases the tumor growth of PIK3CA-mutated cells in vivo. A) NSG mice supplemented with $17 \beta$-estradiol pellets were injected with MCF7-PIK3CA cells $\left(10 \times 10^{6}\right)$ suspended in matrigel and divided into the indicated treatment groups $(n=5)$. The tumor sizes were measured every 4 days for 36 days (the end of treatment). B-E) Representative images and $\mathrm{H}$ scores of IHC staining for Ki67 and P21 expression in MCF7-PIK3CA xenograft tumors. Scale bars are $100 \mu \mathrm{m}$.

\subsubsection{DDR1 inhibition induces cell cycle arrest and synergizes with} palbociclib to suppress tumor growth in vivo

To profile cell signaling changes in response to the treatment combination of palbociclib and 7rh, we performed RPPA analysis of PIK3CA tumor xenografts. Several altered key signaling molecules in cancer were analyzed by using Student's 
$t$-test (Figure 28A). Consistent with the in vitro results, several cell signaling-related proteins (PLK1, pRB-S807/811, cyclin B1, and FoxM1) were significantly downregulated in response to the combination of palbociclib plus 7rh (Figure 28B). Together, these results indicate that DDR1 inhibition induces cell cycle arrest and synergizes with a low dose of palbociclib to suppress tumor growth in vivo.
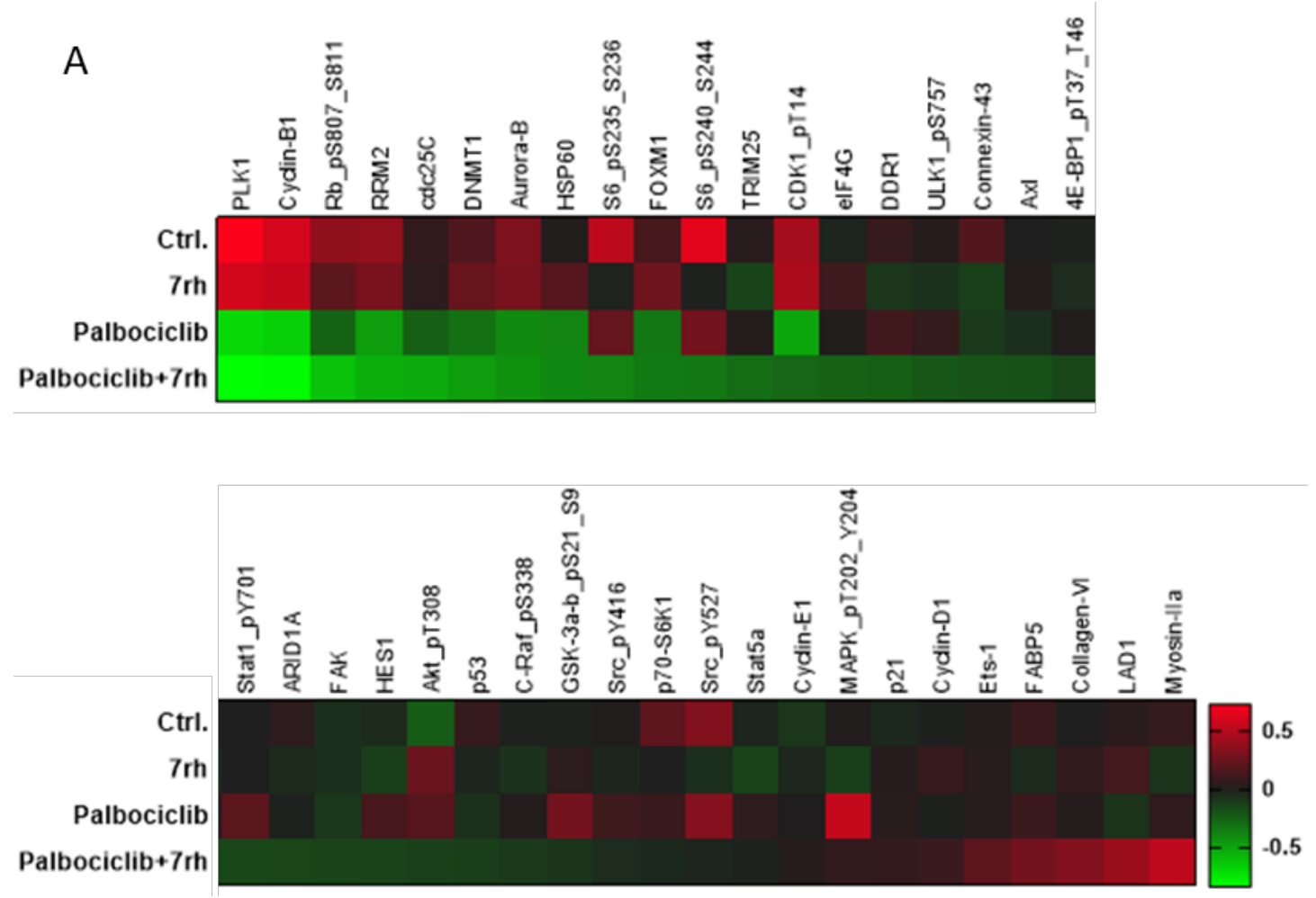


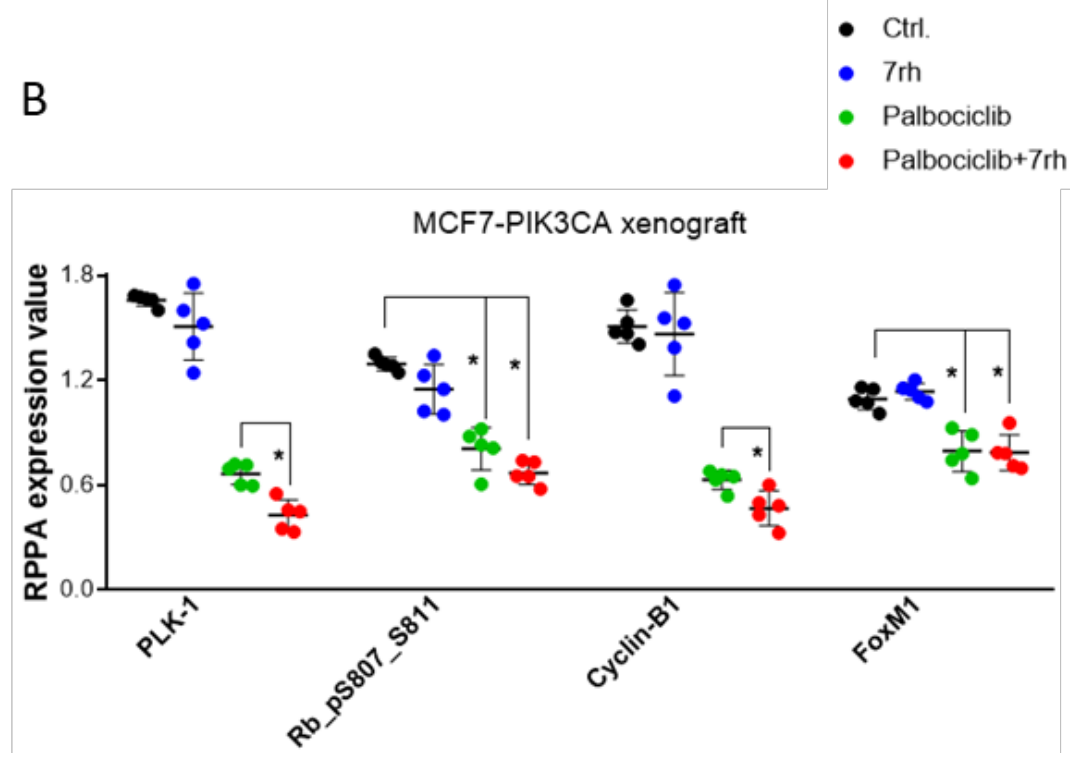

Figure 28. DDR1 inhibition induces cell cycle arrest and synergizes with palbociclib to suppress tumor growth in vivo. A) The heat-map of selected signaling proteins revealed by the RPPA analysis of MCF7-PIK3CA tumor tissues in different treatment groups. B) RPPA statistical analysis of the cell-cycle associated proteins. All data mean \pm SD are indicated using triplicate samples of each representative experiment. P-value calculated based on ANOVA: 0.1234 (ns), $\left.0.0332\left(^{*}\right), 0.0021{ }^{(* *}\right), 0.0002\left(^{(* *}\right)$ and $<0.0001\left(^{* \star \star *}\right)$.

\subsection{SUMMARY}

These results indicate that the combination of palbociclib and 7rh significantly enhances the treatment's antitumor efficacy in vivo, compared with either of these agents used as a single treatment. RPPA analysis of PIK3CA tumor xenografts showed that PLK1, pRB-S807/811, cyclin B1, and FoxM1 proteins were considerably downregulated in response to the combination of palbociclib and 7rh 
(Figure 28B). Consistent with the in vitro results, levels of PLK1 protein (master regulator of the G2-M phase) and cyclin B1 decreased significantly in response to combined treatment, compared with single-agent treatment and controls (palbociclib plus 7rh vs palbociclib alone: $P=0.01)$. A recent next-generation transcriptomic study in ER-positive palbociclib-sensitive and palbociclib-resistant MCF7 cells identified PLK1 deregulation as a novel potential mechanism of resistance to palbociclib [203]. Importantly, upregulation of PLK1 has been reported in tamoxifen-resistant ERpositive breast cancer cells, suggesting that PLK1 inhibition suppresses cell proliferation and in vivo tumor growth [204]. Accordingly, PLK1 may play a major role in driving palbociclib resistance in ER-positive, PIK3CA-mutated breast cancer.

Together, these results show that DDR1 inhibition induces cell cycle arrest and synergizes with a low dose of palbociclib, potentially through targeting PLK1 and cyclin B1 to suppress tumor growth in vivo. Our data suggest that DDR1 targeted therapy combined with CDK4/6 inhibition can augment palbociclib efficacy in ERpositive breast cancer with PI3K pathway activation. 


\section{CHAPTER 9: DISCUSSION AND FUTURE DIRECTIONS}

\subsection{DISCUSSION}

Clinical advances in the last decades have significantly improved outcomes for patients with HR-positive breast cancer. FDA-approved CDK4/6 inhibitors have doubled the time to disease progression when they are added to endocrine treatment for ER-positive, HER2-negative advanced breast cancer. Even so, intrinsic or acquired resistance to these therapeutic agents appears to be inevitable, and understanding the mechanisms underlying this condition has proven challenging. Initial studies implicated PI3K/AKT signaling, the most common activated oncogenic pathway in ER-positive breast cancer, in the blocking of apoptosis. However, more recent evidence has also associated this pathway with the induction of cell cycle progression. The pathway has been shown to be upregulated in response to chronic exposure to CDK4/6 inhibitors, which in turn upregulates cyclin D1 to drive cell cycle progression in the absence of CDK4 and 6 [116]. Further, AKT, as the primary mediator of PI3K signaling, regulates the stability of cyclin-dependent kinase inhibitors p21Cip1 and p27Waf1, leading to DNA synthesis and increased cellular proliferation $[205,206]$.

In this series of studies, we performed a human kinome shRNA library screening with ER-positive HER2-negative isogenic MCF7 breast cell lines to identify the genes that, when targeted, sensitize PIK3CA and AKT1 mutant cells to CDK4/6 inhibitors. The use of isogenic cell lines is a complementary approach to study the influence of genotype on drug sensitivity, as it eliminates the effect of other 
alterations that could impact sensitivity. To date, none of the identified human breast cancer cell lines have an AKT1 mutation. Consequently, the use of the AKT1 knockin model helps us better assess the effects of this mutation.

This screening revealed 25-35 palbociclib synthetic lethality hits. Although most of the candidate genes were involved in a variety of cellular growth and signal transduction pathways, the screening revealed $D D R 1$ as the only novel target that confirmed synthetic lethal interaction with palbociclib in PIK3CA and AKT1 mutant cell lines. To date, the role of DDR1 in cancer biology has been relatively unknown; however, a previous report suggested that DDR1 promotes invasion in breast carcinoma and enhances the chemoresistance [207]. A recent study revealed that in non-small cell lung cancer, co-inhibition of DDR1 and cell-surface transmembrane $4 \mathrm{~L}$ six family member 1 (TM4SF1) causes synergistic reduction of cell proliferation and tumor growth [208]. Moreover, dual pharmacological inhibition of DDR1 (with 7rh) and Notch pathways hampers the growth of KRAS-mutant lung adenocarcinoma [165].

Although we found that ER-positive breast cancers with PIK3CA and AKT1 mutations are less sensitive to palbociclib monotherapy in vitro in an isogenic panel, this association has not been reported clinically. AKT1 mutations are rare, found in only $4 \%$ of breast cancers, and to date the specific efficacy of CDK4/6 inhibitors has not been reported in this genotype. PIK3CA mutations are relatively common in HRpositive breast cancer, yet there is no clear association between PIK3CA mutational status and resistance to CDK4/6 inhibitors in clinical trials examining the combination of endocrine therapy with CDK4/6 inhibitors. In the Phase III 
MONALEESA-2 trial (NCT 01958021), the combination of the CDK4/6 inhibitor ribociclib plus letrozole was compared with placebo plus letrozole for first-line treatment in postmenopausal women with HR-positive, HER2-negative advanced breast cancer [104]. The benefit from ribociclib was maintained irrespective of PIK3CA mutation status. Similarly, in the Phase III PALOMA-3 clinical trial, premenopausal and postmenopausal women with HR-positive, HER2-negative metastatic breast cancer were randomized to fulvestrant in combination with either palbociclib or placebo; PIK3CA mutations were detected in the plasma DNA of the 129 (33\%) of 395 patients for whom these data were available; PIK3CA status did not significantly affect treatment response [97]. Tumor PIK3CA mutation rates were lower among long-term responders in both arms [209]. Circulating tumor DNA sequencing performed at baseline and end-of-treatment for 195 patients in the PALOMA-3 trial showed that new driver mutations emerged in PIK3CA $(p=0.00069)$ and ESR1 after treatment in both arms [210]. Taken together, these data suggest that further research is needed to determine the impact of PIK3CA/AKT1 mutations on the efficacy of CDK4/6 inhibitors as monotherapy. Emerging data already indicates that PIK3CA mutations may underlie acquired resistance to fulvestrant plus palbociclib, possibly by driving fulvestrant resistance. Accordingly, downregulation of ER to mirror dual endocrine and CDK4/6 inhibitor therapies might be able to better model this approach.

We found that $D D R 1$ genomic suppression and 7rh inhibition alone markedly reduce cell proliferation, regardless of PI3K pathway activation, and caused tumor regression in vivo. We also showed that co-inhibition of CDK4/6 and DDR1 in 
PIK3CA and AKT1 mutant cells caused a synergistic decrease in the cell population of $\mathrm{S}$ and $\mathrm{G} 2 / \mathrm{M}$ phases, accompanied by a reduction in RB phosphorylation and cyclin E2. Although these results indicate that the DDR1 inhibitor 7rh induces cell cycle arrest, the mechanism of this synthetic lethal interaction is unknown. A study in a cohort of TCGA breast cancer patients showed that luminal A and B tumors express significantly higher levels of DDR1 mRNA, compared with basal-like breast tumors [171]. This was consistent with our western blot data, in which we detected a higher DDR1 expression level in ER-positive breast cancer cell lines. It suggests a potential correlation between DDR1 expression and the hormone receptor status of the tumor.

Apart from co-expression with ER, DDR1 is involved in regulating and interacting with various growth factor pathways. It has been predicted that the close proximity of RTKs and activated DDR1 acts as a scaffold molecule for PI3K signaling[211]. Similarly, DDR1 association with an AKT1-protein-complex assembly has been identified as inducing pro-survival AKT signaling [212]. Recently, a novel functional cross-talk between DDR1 and insulin-like growth factor $1 \mathrm{R}$ through the PI3K/AKT/miR-199 circuit has been revealed in breast cancer [143]. The PI3K pathway mediates cell cycle progression through cap-dependent mRNA translation of cyclin-dependent kinases that phosphorylate RB, resulting in its dissociation from E2F [213]. Increased transcriptional activity mediated by the free E2F induced the transcription of cell cycle key regulators, including FoxM1 [214]. As a result, it is possible that DDR1 inhibition via 7rh attenuates the PI3K/AKT-mediated induction of cell cycle. Therefore, we can speculate that cooperative regulation of cell cycle 
pathway induced by DDR1 and PI3K/AKT signaling in the context of ER-positivity might be responsible for the therapeutic interaction of palbociclib and 7rh. In summary, we have evaluated a novel combination of CDK4/6 and DDR1 inhibition in PIK3CA/AKT1 mutant ER-positive breast cancer that provides synergistic inhibition of cell cycle and suppression of tumor growth.

\subsection{FUTURE DIRECTIONS}

CDK4/6 inhibitors in combination with endocrine therapy has become the standard of care for ER-positive, HER2-negative advanced metastatic breast cancer. The FDA-approved CDK4/6 inhibitor palbociclib doubles PFS when added to endocrine treatment for ER-positive, HER2-negative advanced breast cancer. In the studies discussed here, we found that ER-positive breast cancer cell lines with PIK3CA or AKT1 mutations are less sensitive to palbociclib monotherapy.

Additionally, this association needs to be examined in response to estrogen depletion or ER degradation to imitate CDK4/6 inhibitor and endocrine combination therapy used in the clinic. Recently, analysis of tumor DNA from patients enrolled in the PALOMA-3 trial identified PIK3CA-activating mutations as a driver of resistance to palbociclib [215]. Determining the sensitivity of PIK3CA or AKT1 mutated cell lines to endocrine treatments (e.g., tamoxifen, fulvestrant) alone and in combination with palbociclib will mirror a more clinically relevant approach.

Our data showed that targeted therapy combining DDR1 with CDK4/6 inhibition augments palbociclib efficacy in PIK3CA-mutated, ER-positive breast cancer in vitro and in vivo. However, clonal evolutions and the absence of stromal interaction in cultured cells may affect the therapeutic evaluation of targeted 
inhibitors. In addition, intra-tumor heterogeneity and the potential alterations accumulating during tumor development represent limitations that impede the identification of potent oncogenic targets. Therefore, future studies using patientderived xenograft tumor models are required to further assess the therapeutic efficacy of DDR1 and CDK4/6 co-inhibition. We used MCF10A cell lines as ectopic overexpression models to evaluate the functional consequences of various $D D R 1$ mutations. These models lack ER expression and the required PI3K pathwayactivated genetic background to study the pro-survival interaction between DDR1 and PI3K signaling, but may be practical for assessing the transformational ability of $D D R 1$ as an oncogene. Nonetheless, to better understand the molecular mechanisms induced by DDR1 wild-type or mutations, an inducible ectopic expression system that can substantially recapitulate DDR1 endogenous activity is essential.

Higher DDR1 mRNA and protein expressions were observed in ER-positive cell lines with mutated PIK3CA or AKT1 than in wild-type cells (Figures 18A and C). Meanwhile, the analysis of TCGA and METABRIC data from patients with breast cancer revealed a higher DDR1 expression in patients with HR-positive breast cancer than in patients with triple-negative breast cancer (Figures $16 \mathrm{~A}$ and $\mathrm{B}$ ). The tendency toward co-occurrence of high DDR1 expression and PIK3CA or AKT mutations in ER-positive patients was not consistent across different datasets (Figures 16C-E), which may suggest the association of DDR1 with other potential mutations to enhance tumor survival. Intriguingly, a recent study revealed that lung adenocarcinoma tumors and cell lines with EGFR mutations expressed higher DDR1 
level [165]. Therefore, investigating the co-occurrence of DDR1 with alternative driver oncogenes can identify a broader group of ER-positive breast cancer patients who can benefit from DDR1 and CDK4/6 co-targeting. 


\section{BIBLIOGRAPHY}

1. Siegel, R.L., K.D. Miller, and A. Jemal, Cancer Statistics, 2016. Ca-a Cancer Journal for Clinicians, 2016. 66(1): p. 7-30.

2. DeSantis, C.E., R.L. Siegel, A.G. Sauer, K.D. Miller, S.A. Fedewa, K.I. Alcaraz, and A. Jemal, Cancer statistics for African Americans, 2016: Progress and opportunities in reducing racial disparities. Ca-a Cancer Journal for Clinicians, 2016. 66(4): p. 290-308.

3. Ellsworth, R.E., H.L. Blackburn, C.D. Shriver, P. Soon-Shiong, and D.L. Ellsworth, Molecular heterogeneity in breast cancer: State of the science and implications for patient care. Seminars in Cell \& Developmental Biology, 2017. 64: p. 65-72.

4. Vuong, D., P.T. Simpson, B. Green, M.C. Cummings, and S.R. Lakhani, Molecular classification of breast cancer. Virchows Archiv, 2014. 465(1): p. 114.

5. Weigelt, B., H.M. Horlings, B. Kreike, M.M. Hayes, M. Hauptmann, L.F.A. Wessels, D. de Jong, M.J.V. de Vijver, L.J. Van't Veer, and J.L. Peterse, Refinement of breast cancer classification by molecular characterization of histological special types. Journal of Pathology, 2008. 216(2): p. 141-150.

6. Taherian-Fard, A., S. Srihari, and M.A. Ragan, Breast cancer classification: linking molecular mechanisms to disease prognosis. Briefings in Bioinformatics, 2015. 16(3): p. 461-474. 
7. Bleyer, A. and H.G. Welch, Effect of Three Decades of Screening Mammography on Breast-Cancer Incidence. New England Journal of Medicine, 2012. 367(21): p. 1998-2005.

8. Bloom, H.J.G. and W.W. Richardson, Histological Grading and Prognosis in Breast Cancer - a Study of 1409 Cases of Which 359 Have Been Followed for 15 Years. British Journal of Cancer, 1957. 11(3): p. 359-\&.

9. Rakha, E.A. and I.O. Ellis, Modern Classification of Breast Cancer: Should we Stick With Morphology or Convert to Molecular Profile Characteristics. Advances in Anatomic Pathology, 2011. 18(4): p. 255-267.

10. Rakha, E.A., J.S. Reis, and I.O. Ellis, Combinatorial biomarker expression in breast cancer. Breast Cancer Research and Treatment, 2010. 120(2): p. 293308.

11. Tsang, J.Y.S. and G.M. Tse, Molecular Classification of Breast Cancer. Advances in Anatomic Pathology, 2020. 27(1): p. 27-35.

12. Nielsen, T.O., M.C.U. Cheang, S.K. Chia, D. Voduc, D.X. Gao, S. Leung, P.S. Bernard, C.M. Perou, and M.J. Ellis, Re: Ki67 Index, HER2 Status, and Prognosis of Patients With Luminal B Breast Cancer. Journal of the National Cancer Institute, 2009. 101(24): p. 1730-1730.

13. Bardou, V.J., G. Arpino, R.M. Elledge, C.K. Osborne, and G.M. Clark, Progesterone receptor status significantly improves outcome prediction over estrogen receptor status alone for adjuvant endocrine therapy in two large breast cancer databases. Journal of Clinical Oncology, 2003. 21(10): p. 19731979. 
14. Harvey, J.M., G.M. Clark, C.K. Osborne, and D.C. Allred, Estrogen receptor status by immunohistochemistry is superior to the ligand-binding assay for predicting response to adjuvant endocrine therapy in breast cancer. Journal of Clinical Oncology, 1999. 17(5): p. 1474-1481.

15. Bauer, K.R., M. Brown, R.D. Cress, C.A. Parise, and V. Caggiano, Descriptive analysis of estrogen receptor (ER)negative, progesterone receptor (PR)-negative, and HER2-negative invasive breast cancer, the socalled triple-negative phenotype - A population-based study from the California Cancer Registry. Cancer, 2007. 109(9): p. 1721-1728.

16. Cadoo, K.A., M.N. Fornier, and P.G. Morris, Biological subtypes of breast cancer: current concepts and implications for recurrence patterns. Quarterly Journal of Nuclear Medicine and Molecular Imaging, 2013. 57(4): p. 312-321.

17. Dent, R., M. Trudeau, K.I. Pritchard, W.M. Hanna, H.K. Kahn, C.A. Sawka, L.A. Lickley, E. Rawlinson, P. Sun, and S.A. Narod, Triple-negative breast cancer: Clinical features and patterns of recurrence. Clinical Cancer Research, 2007. 13(15): p. 4429-4434.

18. Pommier, R.M., A. Sanlaville, L. Tonon, J. Kielbassa, E. Thomas, A. Ferrari, A.S. Sertier, F. Hollande, P. Martinez, A. Tissier, A.P. Morel, M. Ouzounova, and A. Puisieux, Comprehensive characterization of claudin-low breast tumors reflects the impact of the cell-of-origin on cancer evolution. Nature Communications, 2020. 11(1).

19. Prat, A., J.S. Parker, O. Karginova, C. Fan, C. Livasy, J.I. Herschkowitz, X.P. He, and C.M. Perou, Phenotypic and molecular characterization of the 
claudin-low intrinsic subtype of breast cancer. Breast Cancer Research, 2010. 12(5).

20. Smid, M., Y.X. Wang, Y. Zhang, A.M. Sieuwerts, J. Yu, J.G.M. Klijn, J.A. Foekens, and J.W.M. Martens, Subtypes of breast cancer show preferential site of relapse. Cancer Research, 2008. 68(9): p. 3108-3114.

21. Perou, C.M., T. Sorlie, M.B. Eisen, M. van de Rijn, S.S. Jeffrey, C.A. Rees, J.R. Pollack, D.T. Ross, H. Johnsen, L.A. Akslen, O. Fluge, A. Pergamenschikov, C. Williams, S.X. Zhu, P.E. Lonning, A.L. Borresen-Dale, P.O. Brown, and D. Botstein, Molecular portraits of human breast tumours. Nature, 2000. 406(6797): p. 747-752.

22. Nik-Zainal, S., H. Davies, J. Staaf, M. Ramakrishna, Dominik, X.Q. Zou, I. Martincorena, L.B. Alexandrov, S. Martin, D.C. Wedge, P. Van Loo, Y.S. Ju, M. Smid, A.B. Brinkman, S. Morganella, M.R. Aure, O.C. Lingjaerde, A. Langerod, M. Ringner, S.M. Ahn, S. Boyault, J.E. Brock, A. Broeks, A. Butler, C. Desmedt, L. Dirix, S. Dronov, A. Fatima, J.A. Foekens, M. Gerstung, G.K.J. Hooijer, S.J. Jang, D.R. Jones, H.Y. Kim, T.A. King, S. Krishnamurthy, H.J. Lee, J.Y. Lee, Y.L. Li, S. McLaren, A. Menzies, V. Mustonen, S. O'Meara, I. Pauporte, X. Pivot, C.A. Purdie, K. Raine, K. Ramakrishnan, F.G. Rodriguez-Gonzalez, G. Romieu, A.M. Sieuwerts, P.T. Simpson, R. Shepherd, L. Stebbings, O.A. Stefansson, J. Teague, S. Tommasi, I. Treilleux, G.G. Van den Eynden, P. Vermeulen, A. Vincent-Salomon, L. Yates, C. Caldas, L. van't Veer, A. Tutt, S. Knappskog, B.K.T. Tan, J. Jonkers, A. Borg, N.T. Ueno, C. Sotiriou, A. Viari, P.A. Futreal, P.J. Campbell, 
P.N. Span, S. Van Laere, S.R. Lakhani, J.E. Eyfjord, A.M. Thompson, E. Birney, H.G. Stunnenberg, M.J. Van De Vijver, J.W.M. Martens, A.L. Borresen-Dale, A.L. Richardson, G. Kong, G. Thomas, and M.R. Stratton, Landscape of somatic mutations in 560 breast cancer whole-genome sequences. Nature, 2016. 534(7605): p. 47-+.

23. Pereira, B., S.F. Chin, O.M. Rueda, H.K.M. Vollan, E. Provenzano, H.A. Bardwell, M. Pugh, L. Jones, R. Russell, S.J. Sammut, D.W.Y. Tsui, B. Liu, S.J. Dawson, J. Abraham, H. Northen, J.F. Peden, A. Mukherjee, G. Turashvili, A.R. Green, S. McKinney, A. Oloumi, S. Shah, N. Rosenfeld, L. Murphy, D.R. Bentley, I.O. Ellis, A. Purushotham, S.E. Pinder, A.L. BorresenDale, H.M. Earl, P.D. Pharoah, M.T. Ross, S. Aparicio, and C. Caldas, The somatic mutation profiles of 2,433 breast cancers refines their genomic and transcriptomic landscapes. Nature Communications, 2016. 7.

24. Koboldt, D.C., R.S. Fulton, M.D. McLellan, H. Schmidt, J. Kalicki-Veizer, J.F. McMichael, L.L. Fulton, D.J. Dooling, L. Ding, E.R. Mardis, R.K. Wilson, A. Ally, M. Balasundaram, Y.S.N. Butterfield, R. Carlsen, C. Carter, A. Chu, E. Chuah, H.-J.E. Chun, R.J.N. Coope, N. Dhalla, R. Guin, C. Hirst, M. Hirst, R.A. Holt, D. Lee, H.I. Li, M. Mayo, R.A. Moore, A.J. Mungall, E. Pleasance, A. Gordon Robertson, J.E. Schein, A. Shafiei, P. Sipahimalani, J.R. Slobodan, D. Stoll, A. Tam, N. Thiessen, R.J. Varhol, N. Wye, T. Zeng, Y. Zhao, I. Birol, S.J.M. Jones, M.A. Marra, A.D. Cherniack, G. Saksena, R.C. Onofrio, N.H. Pho, S.L. Carter, S.E. Schumacher, B. Tabak, B. Hernandez, J. Gentry, H. Nguyen, A. Crenshaw, K. Ardlie, R. Beroukhim, W. Winckler, G. 
Getz, S.B. Gabriel, M. Meyerson, L. Chin, P.J. Park, R. Kucherlapati, K.A. Hoadley, J. Todd Auman, C. Fan, Y.J. Turman, Y. Shi, L. Li, M.D. Topal, X. He, H.-H. Chao, A. Prat, G.O. Silva, M.D. Iglesia, W. Zhao, J. Usary, J.S. Berg, M. Adams, J. Booker, J. Wu, A. Gulabani, T. Bodenheimer, A.P. Hoyle, J.V. Simons, M.G. Soloway, L.E. Mose, S.R. Jefferys, S. Balu, J.S. Parker, D. Neil Hayes, C.M. Perou, S. Malik, S. Mahurkar, H. Shen, D.J. Weisenberger, T. Triche Jr, P.H. Lai, M.S. Bootwalla, D.T. Maglinte, B.P. Berman, D.J. Van Den Berg, S.B. Baylin, P.W. Laird, C.J. Creighton, L.A. Donehower, G. Getz, M. Noble, D. Voet, G. Saksena, N. Gehlenborg, D. DiCara, J. Zhang, H. Zhang, C.-J. Wu, S. Yingchun Liu, M.S. Lawrence, L. Zou, A. Sivachenko, P. Lin, P. Stojanov, R. Jing, J. Cho, R. Sinha, R.W. Park, M.-D. Nazaire, J. Robinson, H. Thorvaldsdottir, J. Mesirov, P.J. Park, L. Chin, S. Reynolds, R.B. Kreisberg, B. Bernard, R. Bressler, T. Erkkila, J. Lin, V. Thorsson, W. Zhang, I. Shmulevich, G. Ciriello, N. Weinhold, N. Schultz, J. Gao, E. Cerami, B. Gross, A. Jacobsen, R. Sinha, B. Arman Aksoy, Y. Antipin, B. Reva, R. Shen, B.S. Taylor, M. Ladanyi, C. Sander, P. Anur, P.T. Spellman, Y. Lu, W. Liu, R.R.G. Verhaak, G.B. Mills, R. Akbani, N. Zhang, B.M. Broom, T.D. Casasent, C. Wakefield, A.K. Unruh, K. Baggerly, K. Coombes, J.N. Weinstein, D. Haussler, C.C. Benz, J.M. Stuart, S.C. Benz, J. Zhu, C.C. Szeto, G.K. Scott, C. Yau, E.O. Paull, D. Carlin, C. Wong, A. Sokolov, J. Thusberg, S. Mooney, S. Ng, T.C. Goldstein, K. Ellrott, M. Grifford, C. Wilks, S. Ma, B. Craft, C. Yan, Y. Hu, D. Meerzaman, J.M. Gastier-Foster, J. Bowen, N.C. Ramirez, A.D. Black, R.E. Pyatt, P. White, E.J. Zmuda, J. Frick, T.M. 
Lichtenberg, R. Brookens, M.M. George, M.A. Gerken, H.A. Harper, K.M. Leraas, L.J. Wise, T.R. Tabler, C. McAllister, T. Barr, M. Hart-Kothari, K. Tarvin, C. Saller, G. Sandusky, C. Mitchell, M.V. lacocca, J. Brown, B. Rabeno, C. Czerwinski, N. Petrelli, O. Dolzhansky, M. Abramov, O. Voronina, O. Potapova, J.R. Marks, W.M. Suchorska, D. Murawa, W. Kycler, M. Ibbs, K. Korski, A. Spychała, P. Murawa, J.J. Brzeziński, H. Perz, R. Łaźniak, M. Teresiak, H. Tatka, E. Leporowska, M. Bogusz-Czerniewicz, J. Malicki, A. Mackiewicz, M. Wiznerowicz, X. Van Le, B. Kohl, N. Viet Tien, R. Thorp, N. Van Bang, H. Sussman, B. Duc Phu, R. Hajek, N. Phi Hung, T. Viet The Phuong, H. Quyet Thang, K. Zaki Khan, R. Penny, D. Mallery, E. Curley, C. Shelton, P. Yena, J.N. Ingle, F.J. Couch, W.L. Lingle, T.A. King, A. Maria Gonzalez-Angulo, G.B. Mills, M.D. Dyer, S. Liu, X. Meng, M. Patangan, N. The Cancer Genome Atlas, L. Genome sequencing centres: Washington University in St, B.C.C.A. Genome characterization centres, I. Broad, Brigham, H. Women's, S. Harvard Medical, C.H. University of North Carolina, H. University of Southern California/Johns, M. Genome data analysis: Baylor College of, B. Institute for Systems, C. Memorial Sloan-Kettering Cancer, H. Oregon, U. Science, M.D.A.C.C. The University of Texas, S.C.B.I. University of California, Nci, R. Biospecimen core resource: Nationwide Children's Hospital Biospecimen Core, A.-I. Tissue source sites, Christiana, Cureline, C. Duke University Medical, C. The Greater Poland Cancer, Ilsbio, C. International Genomics, C. Mayo, Mskcc and M.D.A.C. Center, 
Comprehensive molecular portraits of human breast tumours. Nature, 2012. 490(7418): p. 61-70.

25. Dietel, E., A. Brobeil, C. Tag, S. Gattenloehner, and M. Wimmer, Effectiveness of EGFR/HER2-targeted drugs is influenced by the dounstream interaction shifts of PTPIP51 in HER2-amplified breast cancer cells. Oncogenesis, 2018. 7.

26. Hayashi, S., T. Sakamoto, A. Inoue, N. Yoshida, Y. Omoto, and Y. Yamaguchi, Estrogen and growth factor signaling pathway: basic approaches for clinical application. Journal of Steroid Biochemistry and Molecular Biology, 2003. 86(3-5): p. 433-442.

27. Kuiper, G.G.J.M., E. Enmark, M. PeltoHuikko, S. Nilsson, and J.A. Gustafsson, Cloning of a novel estrogen receptor expressed in rat prostate and ovary. Proceedings of the National Academy of Sciences of the United States of America, 1996. 93(12): p. 5925-5930.

28. Palmieri, C., G.J. Cheng, S. Saji, M. Zelada-Hedman, A. Warri, Z. Weihua, S. Van Noorden, T. Wahlstrom, R.C. Coombes, M. Warner, and J.A. Gustafsson, Estrogen receptor beta in breast cancer. Endocrine-Related Cancer, 2002. 9(1): p. 1-13.

29. Devin-Leclerc, J., X. Meng, F. Delahaye, P. Leclerc, E.E. Baulieu, and M.G. Catelli, Interaction and dissociation by ligands of estrogen receptor and Hsp90: The antiestrogen RU 58668 induces a protein synthesis-dependent clustering of the receptor in the cytoplasm. Molecular Endocrinology, 1998. 12(6): p. 842-854. 
30. Klinge, C.M., Estrogen receptor interaction with co-activators and corepressors. Steroids, 2000. 65(5): p. 227-251.

31. Carroll, J.S., X.S. Liu, A.S. Brodsky, W. Li, C.A. Meyer, A.J. Szary, J. Eeckhoute, W.L. Shao, E.V. Hestermann, T.R. Geistlinger, E.A. Fox, P.A. Silver, and M. Brown, Chromosome-wide mapping of estrogen receptor binding reveals long-range regulation requiring the forkhead protein FoxA1. Cell, 2005. 122(1): p. 33-43.

32. Wang, L.X., J.S. Brugge, and K.A. Janes, Intersection of FOXO- and RUNX1mediated gene expression programs in single breast epithelial cells during morphogenesis and tumor progression. Proceedings of the National Academy of Sciences of the United States of America, 2011. 108(40): p. E803-E812.

33. ; Available from: $\underline{\text { https://seer.cancer.gov/statfacts/html/breast.html. }}$

34. Howlader, N., S.F. Altekruse, C.I. Li, V.W. Chen, C.A. Clarke, L.A.G. Ries, and K.A. Cronin, US Incidence of Breast Cancer Subtypes Defined by Joint Hormone Receptor and HER2 Status. Jnci-Journal of the National Cancer Institute, 2014. 106(5).

35. Patel, H.K. and T. Bihani, Selective estrogen receptor modulators (SERMs) and selective estrogen receptor degraders (SERDs) in cancer treatment. Pharmacology \& Therapeutics, 2018. 186: p. 1-24.

36. Berrodin, T.J., K.C.N. Chang, B.S. Komm, L.P. Freedman, and S. Nagpal, Differential Biochemical and Cellular Actions of Premarin Estrogens: Distinct Pharmacology of Bazedoxifene-Conjugated Estrogens Combination. Molecular Endocrinology, 2009. 23(1): p. 74-85. 
37. Kharode, Y., P.V.N. Bodine, C.P. Miller, C.R. Lyttle, and B.S. Komm, The Pairing of a Selective Estrogen Receptor Modulator, Bazedoxifene, with Conjugated Estrogens as a New Paradigm for the Treatment of Menopausal Symptoms and Osteoporosis Prevention. Endocrinology, 2008. 149(12): p. 6084-6091.

38. Komm, B.S., A New Approach to Menopausal Therapy: The Tissue Selective Estrogen Complex. Reproductive Sciences, 2008. 15(10): p. 984-992.

39. Mustonen, M.V.J., S. Pyrhonen, and P.L. Kellokumpu-Lehtinen, Toremifene in the treatment of breast cancer. World Journal of Clinical Oncology, 2014. 5(3): p. 393-405.

40. Hu, R., L. Hilakivi-Clarke, and R. Clarke, Molecular mechanisms of tamoxifenassociated endometrial cancer. Oncology Letters, 2015. 9(4): p. 1495-1501.

41. Cuzick, J., T. Powles, U. Veronesi, J. Forbes, R. Edwards, S. Ashley, and P. Boyle, Overview of the main outcomes in breast-cancer prevention trials. Lancet, 2003. 361(9354): p. 296-300.

42. den Hollander, P., M.L. Savage, and P.H. Brown, Targeted therapy for breast cancer prevention. Frontiers in Oncology, 2013. 3.

43. Nilsson, S. and J.A. Gustafsson, Estrogen Receptors: Therapies Targeted to Receptor Subtypes. Clinical Pharmacology \& Therapeutics, 2011. 89(1): p. 44-55.

44. Seoud, M.A.F., J. Johnson, and J.C. Weed, Gynecologic Tumors in Tamoxifen-Treated Women with Breast-Cancer. Obstetrics and Gynecology, 1993. 82(2): p. 165-169. 
45. Benz, C.C., G.K. Scott, J.C. Sarup, R.M. Johnson, D. Tripathy, E. Coronado, H.M. Shepard, and C.K. Osborne, Estrogen-Dependent, Tamoxifen-Resistant Tumorigenic Growth of Mcf-7 Cells Transfected with Her2/Neu. Breast Cancer Research and Treatment, 1992. 24(2): p. 85-95.

46. Fuqua, S.A.W., Estrogen-Receptor Mutagenesis and Hormone Resistance. Cancer, 1994. 74(3): p. 1026-1029.

47. Jordan, V.C., Selective estrogen receptor modulation: Concept and consequences in cancer. Cancer Cell, 2004. 5(3): p. 207-213.

48. McDonnell, D.P., S.E. Wardell, and J.D. Norris, Oral Selective Estrogen Receptor Downregulators (SERDs), a Breakthrough Endocrine Therapy for Breast Cancer. Journal of Medicinal Chemistry, 2015. 58(12): p. 4883-4887.

49. Ellis, M.J., A. Llombart-Cussac, D. Feltl, J.A. Dewar, M. Jasiowka, N. Hewson, Y. Rukazenkov, and J.F.R. Robertson, Fulvestrant 500 mg Versus Anastrozole $1 \mathrm{mg}$ for the First-Line Treatment of Advanced Breast Cancer: Overall Survival Analysis From the Phase II FIRST Study. Journal of Clinical Oncology, 2015. 33(32): p. 3781-+.

50. Robertson, J.F.R., J.P.O. Lindemann, A. Llombart-Cussac, J. Rolski, D. Feltl, J. Dewar, L. Emerson, A. Dean, and M.J. Ellis, Fulvestrant 500 mg versus anastrozole $1 \mathrm{mg}$ for the first-line treatment of advanced breast cancer: follow-up analysis from the randomized 'FIRST' study. Breast Cancer Research and Treatment, 2012. 136(2): p. 503-511. 
51. Hu, X.F., M. Veroni, M. De Luise, A. Wakeling, R. Sutherland, C.K. Watts, and J.R. Zalcberg, Circumvention of tamoxifen resistance by the pure antiestrogen ICI 182,780. Int J Cancer, 1993. 55(5): p. 873-6.

52. Osborne, C.K., M. Jarman, R. Mccague, E.B. Coronado, S.G. Hilsenbeck, and A.E. Wakeling, The Importance of Tamoxifen Metabolism in TamoxifenStimulated Breast-Tumor Growth. Cancer Chemotherapy and Pharmacology, 1994. 34(2): p. 89-95.

53. Simpson, E.R. and S.R. Davis, Minireview. Aromatase and the regulation of estrogen biosynthesis - Some new perspectives. Endocrinology, 2001. 142(11): p. 4589-4594.

54. Miller, W.R., Aromatase-Activity in Breast-Tissue. Journal of Steroid Biochemistry and Molecular Biology, 1991. 39(5b): p. 783-790.

55. Akli, S., T. Bui, H. Wingate, A. Biernacka, S. Moulder, S.L. Tucker, K.K. Hunt, and K. Keyomarsi, Low-molecular-weight cyclin E can bypass letrozoleinduced G1 arrest in human breast cancer cells and tumors. Clin Cancer Res, 2010. 16(4): p. 1179-90.

56. Generali, D., F.M. Buffa, A. Berruti, M.P. Brizzi, L. Campo, S. Bonardi, A. Bersiga, G. Allevi, M. Milani, S. Aguggini, M. Papotti, L. Dogliotti, A. Bottini, A.L. Harris, and S.B. Fox, Phosphorylated ER alpha, HIF-1 alpha, and MAPK Signaling As Predictors of Primary Endocrine Treatment Response and Resistance in Patients With Breast Cancer. Journal of Clinical Oncology, 2009. 27(2): p. 227-234. 
57. Wang, L., K.A. Ellsworth, I. Moon, L.L. Pelleymounter, B.W. Eckloff, Y.N. Martin, B.L. Fridley, G.D. Jenkins, A. Batzler, V.J. Suman, S. Ravi, J.M. Dixon, W.R. Miller, E.D. Wieben, A. Buzdar, R.M. Weinshilboum, and J.N. Ingle, Functional genetic polymorphisms in the aromatase gene CYP19 vary the response of breast cancer patients to neoadjuvant therapy with aromatase inhibitors. Cancer Res, 2010. 70(1): p. 319-28.

58. Polyak, K., Heterogeneity in breast cancer. The Journal of Clinical Investigation, 2011. 121(10): p. 3786-3788.

59. Vanhaesebroeck, B., J. Guillermet-Guibert, M. Graupera, and B. Bilanges, The emerging mechanisms of isoform-specific PI3K signalling. Nat Rev Mol Cell Biol, 2010. 11(5): p. 329-41.

60. Gray, C.W. and A.C.F. Coster, Crosstalk in transition: the translocation of Akt. J Math Biol, 2018.

61. O'Neill, A.K., M.J. Niederst, and A.C. Newton, Suppression of survival signalling pathways by the phosphatase PHLPP. FEBS J, 2013. 280(2): p. $572-83$.

62. Gewinner, C., Z.G.C. Wang, A. Richardson, J. Teruya-Feldstein, D. Etemadmoghadam, D. Bowtell, J. Barretina, W.M. Lin, L. Rameh, L. Salmena, P.P. Pandolfi, and L.C. Cantley, Evidence that Inositol Polyphosphate 4Phosphatase Type II Is a Tumor Suppressor that Inhibits PI3K Signaling. Cancer Cell, 2009. 16(2): p. 115-125. 
63. Altomare, D.A. and A.R. Khaled, Homeostasis and the Importance for a Balance Between AKT/mTOR Activity and Intracellular Signaling. Current Medicinal Chemistry, 2012. 19(22): p. 3748-3762.

64. Jacinto, E., V. Facchinetti, D. Liu, N. Soto, S.N. Wei, S.Y. Jung, Q.J. Huang, J. Qin, and B. Su, SIN1/MIP1 maintains rictor-mTOR complex integrity and regulates Akt phosphorylation and substrate specificity. Cell, 2006. 127(1): p. 125-137.

65. Li, X.J., S.H. Leem, M.H. Park, and S.M. Kim, Regulation of YAP through an Akt-dependent process by 3, 3 '-diindolylmethane in human colon cancer cells. International Journal of Oncology, 2013. 43(6): p. 1992-1998.

66. Xu, Q., L.Z. Liu, X. Qian, Q. Chen, Y. Jiang, D. Li, L.H. Lai, and B.H. Jiang, MiR-145 directly targets p70S6K1 in cancer cells to inhibit tumor growth and angiogenesis. Nucleic Acids Research, 2012. 40(2): p. 761-774.

67. Datta, S.R., H. Dudek, X. Tao, S. Masters, H.A. Fu, Y. Gotoh, and M.E. Greenberg, Akt phosphorylation of BAD couples survival signals to the cellintrinsic death machinery. Cell, 1997. 91(2): p. 231-241.

68. Qi, X.J., G.M. Wildey, and P.H. Howe, Evidence that $\operatorname{Ser}(87)$ of $\operatorname{Bim}(E L)$ is phosphorylated by Akt and regulates BimEL apoptotic function. Journal of Biological Chemistry, 2006. 281(2): p. 813-823.

69. Sangawa, A., M. Shintani, N. Yamao, and S. Kamoshida, Phosphorylation status of Akt and caspase-9 in gastric and colorectal carcinomas. International Journal of Clinical and Experimental Pathology, 2014. 7(6): p. 3312-3317. 
70. Zhang, X.B., N.M. Tang, T.J. Hadden, and A.K. Rishi, Akt, FoxO and regulation of apoptosis. Biochimica Et Biophysica Acta-Molecular Cell Research, 2011. 1813(11): p. 1978-1986.

71. Ogawara, Y., S. Kishishita, T. Obata, Y. Isazawa, T. Suzuki, K. Tanaka, N. Masuyama, and Y. Gotoh, Akt enhances Mdm2-mediated ubiquitination and degradation of p53. Journal of Biological Chemistry, 2002. 277(24): p. 2184321850.

72. Park, S., D. Kim, H.C. Dan, H.H. Chen, J.R. Testa, and J.Q. Cheng, Identification of Akt Interaction Protein PHF20/TZP That Transcriptionally Regulates p53. Journal of Biological Chemistry, 2012. 287(14): p. 1115111163.

73. Park, S., D. Kim, H.C. Dan, H.H. Chen, J.R. Testa, and J.Q. Cheng, Identification of Akt interaction protein PHF20/TZP that transcriptionally regulates p53 (vol 287, pg 11151, 2012). Journal of Biological Chemistry, 2016. 291(43): p. 22852-22852.

74. Laplante, M. and D.M. Sabatini, mTOR signaling in growth control and disease. Cell, 2012. 149(2): p. 274-93.

75. Yuan, T.L. and L.C. Cantley, PI3K pathway alterations in cancer: variations on a theme. Oncogene, 2008. 27(41): p. 5497-510.

76. Jiang, B.H. and L.Z. Liu, PI3K/PTEN signaling in angiogenesis and tumorigenesis. Adv Cancer Res, 2009. 102: p. 19-65.

77. Dey, N., De, Pradip, Leyland-Jones, PI3K-mTOR in Cancer and Cancer Therapy. 2016. 
78. Bellacosa, A., D. de Feo, A.K. Godwin, D.W. Bell, J.Q. Cheng, D.A. Altomare, M. Wan, L. Dubeau, G. Scambia, V. Masciullo, G. Ferrandina, P. Benedetti Panici, S. Mancuso, G. Neri, and J.R. Testa, Molecular alterations of the AKT2 oncogene in ovarian and breast carcinomas. Int J Cancer, 1995. 64(4): p. 280-5.

79. Dahl, E., A. Sadr-Nabavi, E. Klopocki, B. Betz, S. Grube, R. Kreutzfeld, M. Himmelfarb, H.X. An, S. Gelling, I. Klaman, B. Hinzmann, G. Kristiansen, R. Grutzmann, R. Kuner, B. Petschke, K. Rhiem, K. Wiechen, C. Sers, O. Wiestler, A. Schneider, H. Hofler, J. Nahrig, M. Dietel, R. Schafer, A. Rosenthal, R. Schmutzler, M. Durst, A. Meindl, and D. Niederacher, Systematic identification and molecular characterization of genes differentially expressed in breast and ovarian cancer. Journal of Pathology, 2005. 205(1): p. 21-28.

80. Andre, F., E.M. Ciruelos, G. Rubovszky, M. Campone, S. Loibl, H.S. Rugo, H. Iwata, P. Conte, I.A. Mayer, B. Kaufman, T. Yamashita, Y.S. Lu, K. Inoue, M. Takahashi, Z. Papai, A.S. Longin, D. Mills, C. Wilke, S. Hirawat, and D. Juric, Alpelisib (ALP) 1 fulvestrant (FUL) for advanced breast cancer (ABC): Results of the phase III SOLAR-1 trial. Annals of Oncology, 2018. 29: p. 709-709.

81. Bhutani, J., A. Sheikh, and A.K. Niazi, Akt inhibitors: mechanism of action and implications for anticancer therapeutics. Infectious Agents and Cancer, 2013.

\section{8.}

82. Fortier, A.M., E. Asselin, and M. Cadrin, Functional specificity of Akt isoforms in cancer progression. Biomol Concepts, 2011. 2(1-2): p. 1-11. 
83. Hyman, D.M., L.M. Smyth, M.T.A. Donoghue, S.N. Westin, P.L. Bedard, E.J. Dean, H. Bando, A.B. El-Khoueiry, J.A. Perez-Fidalgo, A. Mita, J.H.M. Schellens, M.T. Chang, J.B. Reichel, N. Bouvier, S.D. Selcuklu, T.E. Soumerai, J. Torrisi, J.P. Erinjeri, H. Ambrose, J.C. Barrett, B. Dougherty, A. Foxley, J.P.O. Lindemann, R. McEwen, M. Pass, G. Schiavon, M.F. Berger, S. Chandarlapaty, D.B. Solit, U. Banerji, J. Baselga, and B.S. Taylor, AKT Inhibition in Solid Tumors With AKT1 Mutations. J Clin Oncol, 2017. 35(20): p. 2251-2259.

84. Hanahan, D. and R.A. Weinberg, Hallmarks of Cancer: The Next Generation. Cell, 2011. 144(5): p. 646-674.

85. Malumbres, M., Cyclin-dependent kinases. Genome Biology, 2014. 15(6).

86. Malumbres, M. and M. Barbacid, Cell cycle, CDKs and cancer: a changing paradigm. Nature Reviews Cancer, 2009. 9(3): p. 153-166.

87. Jackson, S.P. and J. Bartek, The DNA-damage response in human biology and disease. Nature, 2009. 461(7267): p. 1071-1078.

88. Yu, Q.Y., Y. Geng, and P. Sicinski, Specific protection against breast cancers by cyclin D1 ablation. Nature, 2001. 411(6841): p. 1017-1021.

89. Yu, Q.Y., E. Sicinska, Y. Geng, M. Ahnstrom, A. Zagozdzon, Y.X. Kong, H. Gardner, H. Kiyokawa, L.N. Harris, O. Stal, and P. Sicinski, Requirement for CDK4 kinase function in breast cancer. Cancer Cell, 2006. 9(1): p. 23-32.

90. Koboldt, D.C., R.S. Fulton, M.D. McLellan, H. Schmidt, J. Kalicki-Veizer, J.F. McMichael, L.L. Fulton, D.J. Dooling, L. Ding, E.R. Mardis, R.K. Wilson, A. Ally, M. Balasundaram, Y.S.N. Butterfield, R. Carlsen, C. Carter, A. Chu, E. 
Chuah, H.J.E. Chun, R.J.N. Coope, N. Dhalla, R. Guin, C. Hirst, M. Hirst, R.A. Holt, D. Lee, H.Y.I. Li, M. Mayo, R.A. Moore, A.J. Mungall, E. Pleasance, A.G. Robertson, J.E. Schein, A. Shafiei, P. Sipahimalani, J.R. Slobodan, D. Stoll, A. Tam, N. Thiessen, R.J. Varhol, N. Wye, T. Zeng, Y.J. Zhao, I. Birol, S.J.M. Jones, M.A. Marra, A.D. Cherniack, G. Saksena, R.C. Onofrio, N.H. Pho, S.L. Carter, S.E. Schumacher, B. Tabak, B. Hernandez, J. Gentry, H. Nguyen, A. Crenshaw, K. Ardlie, R. Beroukhim, W. Winckler, G. Getz, S.B. Gabriel, M. Meyerson, L. Chin, P.J. Park, R. Kucherlapati, K.A. Hoadley, J.T. Auman, C. Fan, Y.J. Turman, Y. Shi, L. Li, M.D. Topal, X.P. He, H.H. Chao, A. Prat, G.O. Silva, M.D. Iglesia, W. Zhao, J. Usary, J.S. Berg, M. Adams, J. Booker, J.Y. Wu, A. Gulabani, T. Bodenheimer, A.P. Hoyle, J.V. Simons, M.G. Soloway, L.E. Mose, S.R. Jefferys, S. Balu, J.S. Parker, D.N. Hayes, C.M. Perou, S. Malik, S. Mahurkar, H. Shen, D.J. Weisenberger, T. Triche, P.H. Lai, M.S. Bootwalla, D.T. Maglinte, B.P. Berman, D.J. Van den Berg, S.B. Baylin, P.W. Laird, C.J. Creighton, L.A. Donehower, G. Getz, M. Noble, D. Voet, G. Saksena, N. Gehlenborg, D. DiCara, J.H. Zhang, H.L. Zhang, C.J. Wu, S.Y. Liu, M.S. Lawrence, L.H. Zou, A. Sivachenko, P. Lin, P. Stojanov, R. Jing, J. Cho, R. Sinha, R.W. Park, M.D. Nazaire, J. Robinson, H. Thorvaldsdottir, J. Mesirov, P.J. Park, L. Chin, S. Reynolds, R.B. Kreisberg, B. Bernard, R. Bressler, T. Erkkila, J. Lin, V. Thorsson, W. Zhang, I. Shmulevich, G. Ciriello, N. Weinhold, N. Schultz, J.J. Gao, E. Cerami, B. Gross, A. Jacobsen, R. Sinha, B.A. Aksoy, Y. Antipin, B. Reva, R.L. Shen, B.S. Taylor, M. Ladanyi, C. Sander, P. Anur, P.T. Spellman, Y.L. Lu, W.B. Liu, R.R.G. Verhaak, G.B. 
Mills, R. Akbani, N.X. Zhang, B.M. Broom, T.D. Casasent, C. Wakefield, A.K. Unruh, K. Baggerly, K. Coombes, J.N. Weinstein, D. Haussler, C.C. Benz, J.M. Stuart, S.C. Benz, J.C. Zhu, C.C. Szeto, G.K. Scott, C. Yau, E.O. Paul, D. Carlin, C. Wong, A. Sokolov, J. Thusberg, S. Mooney, S. Ng, T.C. Goldstein, K. Ellrott, M. Grifford, C. Wilks, S. Ma, B. Craft, C.H. Yan, Y. Hu, D. Meerzaman, J.M. Gastier-Foster, J. Bowen, N.C. Ramirez, A.D. Black, R.E. Pyatt, P. White, E.J. Zmuda, J. Frick, T. Lichtenberg, R. Brookens, M.M. George, M.A. Gerken, H.A. Harper, K.M. Leraas, L.J. Wise, T.R. Tabler, C. McAllister, T. Barr, M. Hart-Kothari, K. Tarvin, C. Saller, G. Sandusky, C. Mitchell, M.V. lacocca, J. Brown, B. Rabeno, C. Czerwinski, N. Petrelli, O. Dolzhansky, M. Abramov, O. Voronina, O. Potapova, J.R. Marks, W.M. Suchorska, D. Murawa, W. Kycler, M. Ibbs, K. Korski, A. Spychala, P. Murawa, J.J. Brzezinski, H. Perz, R. Lazniak, M. Teresiak, H. Tatka, E. Leporowska, M. Bogusz-Czerniewicz, J. Malicki, A. Mackiewicz, M. Wiznerowicz, X.V. Le, B. Kohl, N.V. Tien, R. Thorp, N.V. Bang, H. Sussman, B.D. Phu, R. Hajek, N.P. Hung, V.T.P. Tran, H.Q. Thang, K.Z. Khan, R. Penny, D. Mallery, E. Curley, C. Shelton, P. Yena, J.N. Ingle, F.J. Couch, W.L. Lingle, T.A. King, A.M. Gonzalez-Angulo, G.B. Mills, M.D. Dyer, S.Y. Liu, X.L. Meng, M. Patangan, F. Waldman, H. Stoppler, W.K. Rathmell, L. Thorne, M. Huang, L. Boice, A. Hill, C. Morrison, C. Gaudioso, W. Bshara, K. Daily, S.C. Egea, M.D. Pegram, C. Gomez-Fernandez, R. Dhir, R. Bhargava, A. Brufsky, C.D. Shriver, J.A. Hooke, J.L. Campbell, R.J. Mural, H. Hu, S. Somiari, C. Larson, B. Deyarmin, L. Kvecher, A.J. Kovatich, M.J. Ellis, T.A. 
King, H. Hu, F.J. Couch, R.J. Mural, T. Stricker, K. White, O. Olopade, J.N. Ingle, C.Q. Luo, Y.Q. Chen, J.R. Marks, F. Waldman, M. Wiznerowicz, R. Bose, L.W. Chang, A.H. Beck, A.M. Gonzalez-Angulo, T. Pihl, M. Jensen, R. Sfeir, A. Kahn, A. Chu, P. Kothiyal, Z.N. Wang, E. Snyder, J. Pontius, B. Ayala, M. Backus, J. Walton, J. Baboud, D. Berton, M. Nicholls, D. Srinivasan, R. Raman, S. Girshik, P. Kigonya, S. Alonso, R. Sanbhadti, S. Barletta, D. Pot, M. Sheth, J.A. Demchok, K.R.M. Shaw, L.M. Yang, G. Eley, M.L. Ferguson, R.W. Tarnuzzer, J.S. Zhang, L.A.L. Dillon, K. Buetow, P. Fielding, B.A. Ozenberger, M.S. Guyer, H.J. Sofia, J.D. Palchik and C.G.A. Network, Comprehensive molecular portraits of human breast tumours. Nature, 2012. 490(7418): p. 61-70.

91. Platet, N., A.M. Cathiard, M. Gleizes, and M. Garcia, Estrogens and their receptors in breast cancer progression: a dual role in cancer proliferation and invasion. Critical Reviews in Oncology Hematology, 2004. 51(1): p. 55-67.

92. Sherr, C.J., D. Beach, and G.I. Shapiro, Targeting CDK4 and CDK6: From Discovery to Therapy. Cancer Discovery, 2016. 6(4): p. 353-367.

93. Narasimha, A.M., M. Kaulich, G.S. Shapiro, Y.J. Choi, P. Sicinski, and S.F. Dowdy, Cyclin D activates the Rb tumor suppressor by monophosphorylation. Elife, 2014. 3.

94. O'Leary, B., R.S. Finn, and N.C. Turner, Treating cancer with selective CDK4/6 inhibitors. Nature Reviews Clinical Oncology, 2016. 13(7): p. 417430. 
95. Finn, R.S., J. Dering, D. Conklin, O. Kalous, D.J. Cohen, A.J. Desai, C. Ginther, M. Atefi, I. Chen, C. Fowst, G. Los, and D.J. Slamon, PD 0332991, a selective cyclin D kinase 4/6 inhibitor, preferentially inhibits proliferation of luminal estrogen receptor-positive human breast cancer cell lines in vitro. Breast Cancer Research, 2009. 11(5).

96. Malorni, L., G. Curigliano, A.M. Minisini, S. Cinieri, C. Tondini, G. Arpino, L. Pavesi, A. Martignetti, C. Criscitiello, F. Puglisi, M. Pestrin, G. Sanna, E. Moretti, E. Risi, C. Biagioni, L. Boni, D. Baldari, M.E. Buyse, L. Biganzoli, and A. Di Leo, $A$ phase II trial of the CDK4/6 inhibitor palbociclib $(P)$ as single agent or in combination with the same endocrine therapy $(E T)$ received prior to disease progression, in patients (pts) with hormone receptor positive $(\mathrm{HR}+)$ HER2 negative (HER2-) metastatic breast cancer (mBC) (TREnd trial). Journal of Clinical Oncology, 2017. 35.

97. Cristofanilli, M., N.C. Turner, and I. Bondarenko, Fulvestrant plus palbociclib versus fulvestrant plus placebo for treatment of hormone-receptor-positive, HER2-negative metastatic breast cancer that progressed on previous endocrine therapy (PALOMA-3): final analysis of the multicentre, doubleblind, phase 3 randomised controlled trial (vol 17, pg 431, 2016). Lancet Oncology, 2016. 17(7): p. E270-E270.

98. Morikawa, A. and N.L. Henry, Palbociclib for the Treatment of Estrogen Receptor-Positive, HER2-Negative Metastatic Breast Cancer. Clinical Cancer Research, 2015. 21(16): p. 3591-3596. 
99. Messina, C., C. Cattrini, G. Buzzatti, L. Cerbone, E. Zanardi, M. Messina, and F. Boccardo, CDK4/6 inhibitors in advanced hormone receptorpositive/HER2-negative breast cancer: a systematic review and meta-analysis of randomized trials. Breast Cancer Research and Treatment, 2018. 172(1): p. 9-21.

100. Beaver, J.A., L. Amiri-Kordestani, R. Charlab, W. Chen, T. Palmby, A. Tilley, J.F. Zirkelbach, J.Y. Yu, Q. Liu, L. Zhao, J. Crich, X.H. Chen, M. Hughes, E. Bloomquist, S.H. Tang, R. Sridhara, P.G. Kluetz, G. Kim, A. Ibrahim, R. Pazdur, and P. Cortazar, FDA Approval: Palbociclib for the Treatment of Postmenopausal Patients with Estrogen Receptor-Positive, HER2-Negative Metastatic Breast Cancer. Clinical Cancer Research, 2015. 21(21): p. 47604766.

101. Loibl, S., N.C. Turner, J. Ro, M. Cristofanilli, H. Iwata, S.A. Im, N. Masuda, S. Loi, F. Andre, N. Harbeck, S. Verma, E. Folkerd, K.P. Theall, J. Hoffman, K. Zhang, C.H. Bartlett, and M. Dowsett, Palbociclib Combined with Fulvestrant in Premenopausal Women with Advanced Breast Cancer and Prior Progression on Endocrine Therapy: PALOMA-3 Results. Oncologist, 2017. 22(9): p. 1028-1038.

102. Walker, A.J., S. Wedam, L. Amiri-Kordestani, E. Bloomquist, S.G. Tang, R. Sridhara, W. Chen, T.R. Palmby, J.F. Zirkelbach, W.T. Fu, Q. Liu, A. Tilley, G. Kim, P.G. Kluetz, A.E. McKee, and R. Pazdur, FDA Approval of Palbociclib in Combination with Fulvestrant for the Treatment of Hormone Receptor- 
Positive, HER2-Negative Metastatic Breast Cancer. Clinical Cancer Research, 2016. 22(20): p. 4968-4972.

103. Kim, S., A. Loo, R. Chopra, G. Caponigro, A. Huang, S. Vora, S.

Parasuraman, S. Howard, N. Keen, W. Sellers, and C. Brain, Abstract PR02:

LEE011: An orally bioavailable, selective small molecule inhibitor of CDK4/6Reactivating Rb in cancer. Molecular Cancer Therapeutics, 2013. 12(11 Supplement): p. PR02-PR02.

104. Hortobagyi, G.N., S.M. Stemmer, H.A. Burris, Y.S. Yap, G.S. Sonke, S. Paluch-Shimon, M. Campone, K.L. Blackwell, F. Andre, E.P. Winer, W. Janni, S. Verma, P. Conte, C.L. Arteaga, D.A. Cameron, K. Petrakova, L.L. Hart, C. Villanueva, A. Chan, E. Jakobsen, A. Nusch, O. Burdaeva, E.M. Grischke, E. Alba, E. Wist, N. Marschner, A.M. Favret, D. Yardley, T. Bachelot, L.M. Tseng, S. Blau, F. Xuan, F. Souami, M. Miller, C. Germa, S. Hirawat, and J. O'Shaughnessy, Ribociclib as First-Line Therapy for HR-Positive, Advanced Breast Cancer. New England Journal of Medicine, 2016. 375(18): p. 17381748.

105. Pernas, S., S.M. Tolaney, E.P. Winer, and S. Goel, CDK4/6 inhibition in breast cancer: current practice and future directions. Therapeutic Advances in Medical Oncology, 2018. 10.

106. Ribociclib Approved for Advanced Breast Cancer. Cancer Discov, 2017. 7(5): p. Of3.

107. Slamon, D.J., P. Neven, S. Chia, P.A. Fasching, M. De Laurentiis, S.A. Im, K. Petrakova, G.V. Bianchi, F.J. Esteva, M. Martin, A. Nusch, G.S. Sonke, L. De 
la Cruz-Merino, J.T. Beck, X. Pivot, G. Vidam, Y.B. Wang, K.R. Lorenc, M. Miller, T. Taran, and G. Jerusalem, Phase III Randomized Study of Ribociclib and Fulvestrant in Hormone Receptor-Positive, Human Epidermal Growth Factor Receptor 2-Negative Advanced Breast Cancer: MONALEESA-3. Journal of Clinical Oncology, 2018. 36(24): p. 2465-+.

108. Chen, P., N.V. Lee, W.Y. Hu, M.R. Xu, R.A. Ferre, H. Lam, S. Bergqvist, J. Solowiej, W. Diehl, Y.A. He, X. Yu, A. Nagata, T. VanArsdale, and B.W. Murray, Spectrum and Degree of CDK Drug Interactions Predicts Clinical Performance. Molecular Cancer Therapeutics, 2016. 15(10): p. 2273-2281.

109. Gelbert, L.M., S.F. Cai, X. Lin, C. Sanchez-Martinez, M. del Prado, M.J. Lallena, R. Torres, R.T. Ajamie, G.N. Wishart, R.S. Flack, B.L. Neubauer, J. Young, E.M. Chan, P. Iversen, D. Cronier, E. Kreklau, and A. de Dios, Preclinical characterization of the CDK4/6 inhibitor LY2835219: in-vivo cell cycle-dependent/independent anti-tumor activities alone/in combination with gemcitabine. Investigational New Drugs, 2014. 32(5): p. 825-837.

110. Sledge, G.W., M. Toi, P. Neven, J. Sohn, K. Inoue, X. Pivot, O. Burdaeva, M. Okera, N. Masuda, P.A. Kaufman, H. Koh, E.M. Grischke, M. Frenzel, Y. Lin, S. Barriga, I.C. Smith, N. Bourayou, and A. Llombart-Cussac, MONARCH 2: Abemaciclib in Combination With Fulvestrant in Women With HR+/HER2Advanced Breast Cancer Who Had Progressed While Receiving Endocrine Therapy. Journal of Clinical Oncology, 2017. 35(25): p. 2875-+.

111. Johnston, S., M. Martin, A. Di Leo, S.A. Im, A. Awada, T. Forrester, M. Frenzel, M.C. Hardebeck, J. Cox, S. Barriga, M. Toi, H. Iwata, and M.P. 
Goetz, MONARCH 3 final PFS: a randomized study of abemaciclib as initial therapy for advanced breast cancer. Npj Breast Cancer, 2019. 5.

112. Corona, S.P. and D. Generali, Abemaciclib: a CDK4/6 inhibitor for the treatment of HR+/HER2-advanced breast cancer. Drug Design Development and Therapy, 2018. 12: p. 321-330.

113. Toogood, P.L., P.J. Harvey, J.T. Repine, D.J. Sheehan, S.N. VanderWel, H.R. Zhou, P.R. Keller, D.J. McNamara, D. Sherry, T. Zhu, J. Brodfuehrer, C. Choi, M.R. Barvian, and D.W. Fry, Discovery of a potent and selective inhibitor of cyclin-dependent kinase 4/6. Journal of Medicinal Chemistry, 2005. 48(7): p. 2388-2406.

114. Wander, S.A., O. Cohen, X.Q. Gong, G.N. Johnson, J. Buendia-Buendia, M. Lloyd, D. Kim, F. Luo, P.P. Mao, K. Helvie, K. Kowalski, U. Nayar, S. Parsons, R. Martinez, L. Litchfield, X. Ye, C.P. Yu, V. Jansen, L.A. Garraway, E.P. Winer, S.M. Tolaney, N.U. Lin, S. Buchanan, and N. Wagle, The genomic landscape of intrinsic and acquired resistance to cyclin-dependent kinase 4/6 inhibitors (CDK4/6i) in patients with hormone receptor-positive $(H R+) / H E R 2-m e t a s t a t i c ~ b r e a s t ~ c a n c e r(M B C)$. Cancer Research, 2020. 80(4).

115. Miller, T.W., B.N. Rexer, J.T. Garrett, and C.L. Arteaga, Mutations in the phosphatidylinositol 3-kinase pathway: role in tumor progression and therapeutic implications in breast cancer. Breast Cancer Res, 2011. 13(6): p. 224.

116. Herrera-Abreu, M.T., M. Palafox, U. Asghar, M.A. Rivas, R.J. Cutts, I. GarciaMurillas, A. Pearson, M. Guzman, O. Rodriguez, J. Grueso, M. Bellet, J. 
Cortes, R. Elliott, S. Pancholi, C.J. Lord, J. Baselga, M. Dowsett, L.A. Martin, N.C. Turner, and V. Serra, Early Adaptation and Acquired Resistance to CDK4/6 Inhibition in Estrogen Receptor-Positive Breast Cancer (vol 76, pg 2301, 2016). Cancer Research, 2016. 76(19): p. 5907-5907.

117. Jansen, V.M., N.E. Bhola, J.A. Bauer, L. Formisano, K.M. Lee, K.E. Hutchinson, A.K. Witkiewicz, P.D. Moore, M.V. Estrada, V. Sanchez, P.G. Ericsson, M.E. Sanders, P.R. Pohlmann, M.J. Pishvaian, D.A. Riddle, T.C. Dugger, W. Wei, E.S. Knudsen, and C.L. Arteaga, Kinome-Wide RNA Interference Screen Reveals a Role for PDK1 in Acquired Resistance to CDK4/6 Inhibition in ER-Positive Breast Cancer. Cancer Res, 2017. 77(9): p. 2488-2499.

118. Herrera-Abreu, M.T., M. Palafox, U. Asghar, M.A. Rivas, R.J. Cutts, I. GarciaMurillas, A. Pearson, M. Guzman, O. Rodriguez, J. Grueso, M. Bellet, J. Cortes, R. Elliott, S. Pancholi, J. Baselga, M. Dowsett, L.A. Martin, N.C. Turner, and V. Serra, Early Adaptation and Acquired Resistance to CDK4/6 Inhibition in Estrogen Receptor-Positive Breast Cancer. Cancer Research, 2016. 76(8): p. 2301-2313.

119. Cortes, J., S.A. Im, E. Holgado, J.M. Perez-Garcia, P. Schmid, and M. Chavez-MacGregor, The next era of treatment for hormone receptor-positive, HER2-negative advanced breast cancer: Triplet combination-based endocrine therapies. Cancer Treat Rev, 2017. 61: p. 53-60.

120. Juric, D., R. Ismail-Khan, M. Campone, L. Estevez Garcia, C. Becerra, R. Boer, E. Hamilton, I. Mayer, R. Hui, K.I. Lathrop, O. Pagani, S. Asano, S.G. 
Bhansali, V. Zhang, B. Hewes, and P. Munster, Abstract P3-14-01: Phase Ib/II study of ribociclib and alpelisib and letrozole in ER+, HER2-breast cancer: Safety, preliminary efficacy and molecular analysis. Cancer Research, 2016. 76: p. P3-14.

121. Vora, S.R., D. Juric, N. Kim, M. Mino-Kenudson, T. Huynh, C. Costa, E.L. Lockerman, S.F. Pollack, M. Liu, X. Li, J. Lehar, M. Wiesmann, M. Wartmann, Y. Chen, Z.A. Cao, M. Pinzon-Ortiz, S. Kim, R. Schlegel, A. Huang, and J.A. Engelman, CDK 4/6 inhibitors sensitize PIK3CA mutant breast cancer to PI3K inhibitors. Cancer Cell, 2014. 26(1): p. 136-49.

122. Brachmann, S., C. Fritsch, S.M. Maira, and C. Garcia-Echeverria, PI3K and mTOR inhibitors: a new generation of targeted anticancer agents. Curr Opin Cell Biol, 2009. 21(2): p. 194-8.

123. O'Brien, C., J.J. Wallin, D. Sampath, D. GuhaThakurta, H. Savage, E.A. Punnoose, J. Guan, L. Berry, W.W. Prior, L.C. Amler, M. Belvin, L.S. Friedman, and M.R. Lackner, Predictive biomarkers of sensitivity to the phosphatidylinositol 3' kinase inhibitor GDC-0941 in breast cancer preclinical models. Clin Cancer Res, 2010. 16(14): p. 3670-83.

124. Krop, I.E., I.A. Mayer, V. Ganju, M. Dickler, S. Johnston, S. Morales, D.A. Yardley, B. Melichar, A. Forero-Torres, S.C. Lee, R. de Boer, K. Petrakova, S. Vallentin, E.A. Perez, M. Piccart, M. Ellis, E. Winer, S. Gendreau, M. Derynck, M. Lackner, G. Levy, J. Qiu, J. He, and P. Schmid, Pictilisib for oestrogen receptor-positive, aromatase inhibitor-resistant, advanced or metastatic 
breast cancer (FERGI): a randomised, double-blind, placebo-controlled, phase 2 trial. The Lancet Oncology. 17(6): p. 811-821.

125. Piccart, M., G.N. Hortobagyi, M. Campone, K.I. Pritchard, F. Lebrun, Y. Ito, S. Noguchi, A. Perez, H.S. Rugo, I. Deleu, H.A. Burris, 3rd, L. Provencher, P. Neven, M. Gnant, M. Shtivelband, C. Wu, J. Fan, W. Feng, T. Taran, and J. Baselga, Everolimus plus exemestane for hormone-receptor-positive, human epidermal growth factor receptor-2-negative advanced breast cancer: overall survival results from BOLERO-2dagger. Ann Oncol, 2014. 25(12): p. 2357-62.

126. Beaver, J.A., J.P. Gustin, K.H. Yi, A. Rajpurohit, M. Thomas, S.F. Gilbert, D.M. Rosen, B.H. Park, and J. Lauring, PIK3CA and AKT1 Mutations Have Distinct Effects on Sensitivity to Targeted Pathway Inhibitors in an Isogenic Luminal Breast Cancer Model System. Clinical Cancer Research, 2013. 19(19): p. 5413-5422.

127. Dogruluk, T., Y.H. Tsang, M. Espitia, F. Chen, T. Chen, Z. Chong, V. Appadurai, A. Dogruluk, A.K. Eterovic, P.E. Bonnen, C.J. Creighton, K. Chen, G.B. Mills, and K.L. Scott, Identification of Variant-Specific Functions of PIK3CA by Rapid Phenotyping of Rare Mutations. Cancer Res, 2015. 75(24): p. 5341-54.

128. Spandidos, A., X.W. Wang, H.J. Wang, S. Dragnev, T. Thurber, and B. Seed, A comprehensive collection of experimentally validated primers for Polymerase Chain Reaction quantitation of murine transcript abundance. Bmc Genomics, 2008. 9. 
129. Hu, J., X. He, K.A. Baggerly, K.R. Coombes, B.T. Hennessy, and G.B. Mills, Non-parametric quantification of protein lysate arrays. Bioinformatics, 2007. 23(15): p. 1986-94.

130. Ju, Z., W. Liu, P.L. Roebuck, D.R. Siwak, N. Zhang, Y. Lu, M.A. Davies, R. Akbani, J.N. Weinstein, G.B. Mills, and K.R. Coombes, Development of a robust classifier for quality control of reverse-phase protein arrays. Bioinformatics, 2015. 31(6): p. 912-8.

131. Baselga, J., Targeting the phosphoinositide-3 (PI3) kinase pathway in breast cancer. Oncologist, 2011. 16 Suppl 1: p. 12-9.

132. Ciruelos Gil, E.M., Targeting the PIJK/AKT/mTOR pathway in estrogen receptor-positive breast cancer. Cancer Treat Rev, 2014. 40(7): p. 862-71.

133. Michaloglou, C., C. Crafter, R. Siersbaek, O. Delpuech, J.O. Curwen, L.S. Carnevalli, A.D. Staniszewska, U.M. Polanska, A. Cheraghchi-Bashi, M. Lawson, I. Chernukhin, R. McEwen, J.S. Carroll, and S.C. Cosulich, Combined Inhibition of mTOR and CDK4/6 Is Required for Optimal Blockade of E2F Function and Long-term Growth Inhibition in Estrogen Receptorpositive Breast Cancer. Molecular Cancer Therapeutics, 2018. 17(5): p. 908920.

134. Day, P.J., A. Cleasby, I.J. Tickle, M. O'Reilly, J.E. Coyle, F.P. Holding, R.L. McMenamin, J. Yon, R. Chopra, C. Lengauer, and H. Jhoti, Crystal structure of human CDK4 in complex with a D-type cyclin. Proc Natl Acad Sci U S A, 2009. 106(11): p. 4166-70. 
135. Mendoza, M.C., E.E. Er, and J. Blenis, The Ras-ERK and PI3K-mTOR pathways: cross-talk and compensation. Trends in Biochemical Sciences, 2011. 36(6): p. 320-328.

136. Campbell, I.G., S.E. Russell, D.Y. Choong, K.G. Montgomery, M.L. Ciavarella, C.S. Hooi, B.E. Cristiano, R.B. Pearson, and W.A. Phillips, Mutation of the PIK3CA gene in ovarian and breast cancer. Cancer Res, 2004. 64(21): p. 7678-81.

137. De Marco, C., D. Malanga, N. Rinaldo, F. De Vita, M. Scrima, S. Lovisa, L. Fabris, M.V. Carriero, R. Franco, A. Rizzuto, G. Baldassarre, and G. Viglietto, Mutant AKT1-E17K is oncogenic in lung epithelial cells. Oncotarget, 2015. 6(37): p. 39634-39650.

138. Finn, R.S., M. Martin, H.S. Rugo, S.E. Jones, S.A. Im, K.A. Gelmon, N. Harbeck, O.N. Lipatov, J.M. Walshe, S.L. Moulder, E.R. Gauthier, D.R. Lu, S. Randolph, V. Dieras, and D.J. Slamon, PALOMA-2: Primary results from a phase III trial of palbociclib $(P)$ with letrozole $(L)$ compared with letrozole alone in postmenopausal women with ER/HER2 advanced breast cancer (ABC). Journal of Clinical Oncology, 2016. 34(15).

139. Diehl, J.A., M.G. Cheng, M.F. Roussel, and C.J. Sherr, Glycogen synthase kinase 3 beta regulates cyclin D1 proteolysis and subcellular localization. Genes \& Development, 1998. 12(22): p. 3499-3511.

140. Goel, S., Q. Wang, A.C. Watt, S.M. Tolaney, D.A. Dillon, W. Li, S. Ramm, A.C. Palmer, H. Yuzugullu, V. Varadan, D. Tuck, L.N. Harris, K.K. Wong, X.S. Liu, P. Sicinski, E.P. Winer, I.E. Krop, and J.J. Zhao, Overcoming Therapeutic 
Resistance in HER2-Positive Breast Cancers with CDK4/6 Inhibitors. Cancer Cell, 2016. 29(3): p. 255-269.

141. Guarducci, C., M. Bonechi, G. Boccalini, M. Benelli, E. Risi, A. Di Leo, L. Malorni, and I. Migliaccio, Mechanisms of Resistance to CDK4/6 Inhibitors in Breast Cancer and Potential Biomarkers of Response. Breast Care, 2017. 12(5): p. 304-308.

142. Gadiya, M. and G. Chakraborty, Signaling by discoidin domain receptor 1 in cancer metastasis. Cell Adh Migr, 2018. 12(4): p. 315-323.

143. Belfiore, A., R. Malaguarnera, M.L. Nicolosi, R. Lappano, M. Ragusa, A. Morrione, and V. Vella, A novel functional crosstalk between DDR1 and the IGF axis and its relevance for breast cancer. Cell Adhesion \& Migration, 2018. 12(4): p. 305-314.

144. Playford, M.P., R.J. Butler, X.C. Wang, R.M. Katso, I.E. Cooke, and T.S. Ganesan, The genomic structure of discoidin receptor tyrosine kinase. Genome Research, 1996. 6(7): p. 620-627.

145. Shrivastava, A., C. Radziejewski, E. Campbell, L. Kovac, M. McGlynn, T.E. Ryan, S. Davis, M.P. Goldfarb, D.J. Glass, G. Lemke, and G.D. Yancopoulos, An orphan receptor tyrosine kinase family whose members serve as nonintegrin collagen receptors. Molecular Cell, 1997. 1(1): p. 25-34.

146. Valiathan, R.R., M. Marco, B. Leitinger, C.G. Kleer, and R. Fridman, Discoidin domain receptor tyrosine kinases: new players in cancer progression. Cancer and Metastasis Reviews, 2012. 31(1-2): p. 295-321. 
147. Vogel, W.F., R. Abdulhussein, and C.E. Ford, Sensing extracellular matrix: An update on discoidin domain receptor function. Cellular Signalling, 2006. 18(8): p. $1108-1116$.

148. Alves, F., S. Saupe, M. Ledwon, F. Schaub, W. Hiddemann, and W.F. Vogel, Identification of two novel, kinase-deficient variants of discoidin domain receptor 1: differential expression in human colon cancer cell lines. Faseb Journal, 2001. 15(7): p. 1321-1323.

149. Carafoli, F. and E. Hohenester, Collagen recognition and transmembrane signalling by discoidin domain receptors. Biochimica Et Biophysica ActaProteins and Proteomics, 2013. 1834(10): p. 2187-2194.

150. Leitinger, B., Transmembrane Collagen Receptors. Annual Review of Cell and Developmental Biology, Vol 27, 2011. 27: p. 265-290.

151. Nakada, M., D. Kita, L. Teng, I.V. Pyko, T. Watanabe, Y. Hayashi, and J. Hamada, Receptor Tyrosine Kinases: Principles and Functions in Glioma Invasion. Glioma Signaling, 2013. 986: p. 143-170.

152. Ongusaha, P.P., J.I. Kim, L. Fang, T.W. Wong, G.D. Yancopoulos, S.A. Aaronson, and S.W. Lee, p53 induction and activation of DDR1 kinase counteract p53-mediated apoptosis and influence p53 regulation through a positive feedback loop. Embo Journal, 2003. 22(6): p. 1289-1301.

153. Taube, J.H., J.I. Herschkowitz, K. Komurov, A.Y. Zhou, S. Gupta, J. Yang, K. Hartwell, T.T. Onder, P.B. Gupta, K.W. Evans, B.G. Hollier, P.T. Ram, E.S. Lander, J.M. Rosen, R.A. Weinberg, and S.A. Mani, Core epithelial-tomesenchymal transition interactome gene-expression signature is associated 
with claudin-low and metaplastic breast cancer subtypes (vol 107, pg 15449, 2010). Proceedings of the National Academy of Sciences of the United States of America, 2010. 107(44): p. 19132-19132.

154. Vogel, W.F., A. Aszodi, F. Alves, and T. Pawson, Discoidin domain receptor 1 tyrosine kinase has an essential role in mammary gland development. Molecular and Cellular Biology, 2001. 21(8): p. 2906-2917.

155. Vogel, W., G.D. Gish, F. Alves, and T. Pawson, The discoidin domain receptor tyrosine kinases are activated by collagen. Mol Cell, 1997. 1(1): p. 13-23.

156. Juskaite, V., D. Corcoran, and B. Leitinger, Collagen induces activation of DDR1 through lateral dimer association and phosphorylation between dimers. International Journal of Experimental Pathology, 2017. 98(6): p. A3-A3.

157. Wang, C.Z., H.W. Su, Y.C. Hsu, M.R. Shen, and M.J. Tang, A discoidin domain receptor 1/SHP-2 signaling complex inhibits alpha2beta1-integrinmediated signal transducers and activators of transcription 1/3 activation and cell migration. Mol Biol Cell, 2006. 17(6): p. 2839-52.

158. Shintani, Y., Y. Fukumoto, N. Chaika, R. Svoboda, M.J. Wheelock, and K.R. Johnson, Collagen I-mediated up-regulation of $\mathrm{N}$-cadherin requires cooperative signals from integrins and discoidin domain receptor 1. J Cell Biol, 2008. 180(6): p. 1277-89.

159. Yang, G., Q. Li, S. Ren, X. Lu, L. Fang, W. Zhou, F. Zhang, F. Xu, Z. Zhang, R. Zeng, F. Lottspeich, and Z. Chen, Proteomic, functional and motif-based 
analysis of C-terminal Src kinase-interacting proteins. Proteomics, 2009. 9(21): p. 4944-61.

160. Dejmek, J., K. Dib, M. Jonsson, and T. Andersson, Wnt-5a and G-protein signaling are required for collagen-induced DDR1 receptor activation and normal mammary cell adhesion. Int J Cancer, 2003. 103(3): p. 344-51.

161. L'Hote C, G., P.H. Thomas, and T.S. Ganesan, Functional analysis of discoidin domain receptor 1: effect of adhesion on DDR1 phosphorylation. FASEB J, 2002. 16(2): p. 234-6.

162. Kim, H.G., S.Y. Hwang, S.A. Aaronson, A. Mandinova, and S.W. Lee, DDR1 Receptor Tyrosine Kinase Promotes Prosurvival Pathway through Notch1 Activation (Retracted article. See vol. 294, pg. 18950, 2019). Journal of Biological Chemistry, 2011. 286(20): p. 17672-17681.

163. Eswaramoorthy, R., C.K. Wang, W.C. Chen, M.J. Tang, M.L. Ho, C.C. Hwang, H.M. Wang, and C.Z. Wang, DDR1 regulates the stabilization of cell surface E-cadherin and E-cadherin-mediated cell aggregation. J Cell Physiol, 2010. 224(2): p. 387-97.

164. Suh, H.N. and H.J. Han, Collagen I Regulates the Self-Renewal of Mouse Embryonic Stem Cells Through alpha 2 beta 1 Integrin- and DDR1Dependent Bmi-1. Journal of Cellular Physiology, 2011. 226(12): p. 34223432.

165. Ambrogio, C., G. Gomez-Lopez, M. Falcone, A. Vidal, E. Nadal, N. Crosetto, R.B. Blasco, P.J. Fernandez-Marcos, M. Sanchez-Cespedes, X.M. Ren, Z. Wang, K. Ding, M. Hidalgo, M. Serrano, A. Villanueva, D. Santamaria, and M. 
Barbacid, Combined inhibition of DDR1 and Notch signaling is a therapeutic strategy for KRAS-driven lung adenocarcinoma. Nature Medicine, 2016. 22(3): p. 270-277.

166. Heinzelmann-Schwarz, V.A., M. Gardiner-Garden, S.M. Henshall, J. Scurry, R.A. Scolyer, M.J. Davies, M. Heinzelmann, L.H. Kalish, A. Bali, J.G. Kench, L.S. Edwards, P.M. Vanden Bergh, N.F. Hacker, R.L. Sutherland, and P.M. O'Brien, Overexpression of the cell adhesion molecules DDR1, claudin 3, and Ep-CAM in metaplastic ovarian epithelium and ovarian cancer. Clinical Cancer Research, 2004. 10(13): p. 4427-4436.

167. Jin, H., I.H. Ham, H.J. Oh, C.A. Bae, D. Lee, Y.B. Kim, S.Y. Son, Y.J. Chwae, S.U. Han, R.A. Brekken, and H. Hur, Inhibition of Discoidin Domain Receptor 1 Prevents Stroma-Induced Peritoneal Metastasis in Gastric Carcinoma. Molecular Cancer Research, 2018. 16(10): p. 1590-1600.

168. Weiner, H.L., H.Y. Huang, D. Zagzag, H. Boyce, R. Lichtenbaum, and E.B. Ziff, Consistent and selective expression of the discoidin domain receptor-1 tyrosine kinase in human brain tumors. Neurosurgery, 2000. 47(6): p. 14001409.

169. Moon, A., M. Koh, Y. Woo, D. Lee, R. Valiathan, H.Y. Jung, S.Y. Park, Y.N. Kim, H.R.C. Kim, and R. Fridman, Discoidin Domain Receptor (DDR) 1 is a Novel Transcriptional Target of ZEB1 in Breast Epithelial Cells Undergoing $\mathrm{H}$ Ras-Induced Epithelial to Mesenchymal Transition. Faseb Journal, 2015. 29. 
170. Savagner, P., B. Boyer, A.M. Valles, J. Jouanneau, and J.P. Thiery, Modulations of the epithelial phenotype during embryogenesis and cancer progression. Cancer Treat Res, 1994. 71: p. 229-49.

171. Saby, C., G. Collin, M. Sinane, E. Buache, L. Van Gulick, F. Saltel, E. Maquoi, and H. Morjani, DDR1 and MT1-MMP Expression Levels Are Determinant for Triggering BIK-Mediated Apoptosis by 3D Type I Collagen Matrix in Invasive Basal-Like Breast Carcinoma Cells. Frontiers in Pharmacology, 2019. 10.

172. Assent, D., I. Bourgot, B. Hennuy, P. Geurts, A. Noel, J.M. Foidart, and E. Maquoi, A Membrane-Type-1 Matrix Metalloproteinase (MT1-MMP) Discoidin Domain Receptor 1 Axis Regulates Collagen-Induced Apoptosis in Breast Cancer Cells. Plos One, 2015. 10(3).

173. Mata, R., C. Palladino, M.L. Nicolosi, A.R. Lo Presti, R. Malaguarnera, M. Ragusa, D. Sciortino, A. Morrione, M. Maggiolini, V. Vella, and A. Belfiore, IGF-I induces upregulation of DDR1 collagen receptor in breast cancer cells by suppressing MIR-199a-5p through the PI3K/AKT pathway. Oncotarget, 2016. 7(7): p. 7683-7700.

174. Choi, Y.J. and L. Anders, Signaling through cyclin D-dependent kinases. Oncogene, 2014. 33(15): p. 1890-1903.

175. Gordon, G.M. and W. Du, Conserved RB functions in development and tumor suppression. Protein \& Cell, 2011. 2(11): p. 864-878.

176. Saab, R., J.L. Bills, A.P. Miceli, C.M. Anderson, J.D. Khoury, D.W. Fry, F. Navid, P.J. Houghton, and S.X. Skapek, Pharmacologic inhibition of cyclin- 
dependent kinase 4/6 activity arrests proliferation in myoblasts and rhabdomyosarcoma-derived cells. Molecular Cancer Therapeutics, 2006. 5(5): p. 1299-1308.

177. Kethiwale, S., C.M. Borza, E.W. Lowe, A. Pozzi, and J. Meiler, Discoidin domain receptor 1 (DDR1) kinase as target for structure-based drug discovery. Drug Discovery Today, 2015. 20(2): p. 255-261.

178. Roskoski, R., Properties of FDA-approved small molecule protein kinase inhibitors. Pharmacological Research, 2019. 144: p. 19-50.

179. Day, E., B. Waters, K. Spiegel, T. Alnadaf, P.W. Manley, E. Buchdunger, C. Walker, and G. Jarai, Inhibition of collagen-induced discoidin domain receptor 1 and 2 activation by imatinib, nilotinib and dasatinib. European Journal of Pharmacology, 2008. 599(1-3): p. 44-53.

180. Canning, P., L. Tan, K. Chu, S.W. Lee, N.S. Gray, and A.N. Bullock, Structural Mechanisms Determining Inhibition of the Collagen Receptor DDR1 by Selective and Multi-Targeted Type II Kinase Inhibitors. Journal of Molecular Biology, 2014. 426(13): p. 2457-2470.

181. Gao, M.S., L. Duan, J.F. Luo, L.W. Zhang, X.Y. Lu, Y. Zhang, Z. Zhang, Z.C. Tu, Y. Xu, X.M. Ren, and K. Ding, Discovery and Optimization of 3-(2(Pyrazolo[1,5-a]pyrimidin-6-yl)-ethynyl)benzamides as Novel Selective and Orally Bioavailable Discoidin Domain Receptor 1 (DDR1) Inhibitors. Journal of Medicinal Chemistry, 2013. 56(8): p. 3281-3295.

182. Kim, H.G., L. Tan, E.L. Weisberg, F.Y. Liu, P. Canning, H.G. Choi, S. Ezell, Z. Zhao, H. Wu, J.H. Wang, A. Mandinova, A.N. Bullock, Q.S. Liu, S.W. Lee, 
and N.S. Gray, Discovery of a Potent and Selective DDR1 Receptor Tyrosine Kinase Inhibitor (vol 8, pg 2145, 2013). Acs Chemical Biology, 2014. 9(3): p. 840-840.

183. Richters, A., H.D. Nguyen, T. Phan, J.R. Simard, C. Grutter, J. Engel, and D. Rauh, Identification of Type II and III DDR2 Inhibitors. Journal of Medicinal Chemistry, 2014. 57(10): p. 4252-4262.

184. Gao, M., L. Duan, J. Luo, L. Zhang, X. Lu, Y. Zhang, Z. Zhang, Z. Tu, Y. Xu, X. Ren, and K. Ding, Discovery and optimization of 3-(2-(Pyrazolo[1,5a]pyrimidin-6-yl)ethynyl)benzamides as novel selective and orally bioavailable discoidin domain receptor 1 (DDR1) inhibitors. J Med Chem, 2013. 56(8): p. 3281-95.

185. Anders, L., N. Ke, P. Hydbring, Y.J. Choi, H.R. Widlund, J.M. Chick, H. Zhai, M. Vidal, S.P. Gygi, P. Braun, and P. Sicinski, A systematic screen for CDK4/6 substrates links FOXM1 phosphorylation to senescence suppression in cancer cells. Cancer Cell, 2011. 20(5): p. 620-34.

186. Dean, J.L., C. Thangavel, A.K. McClendon, C.A. Reed, and E.S. Knudsen, Therapeutic CDK4/6 inhibition in breast cancer: key mechanisms of response and failure. Oncogene, 2010. 29(28): p. 4018-32.

187. Franco, J., A.K. Witkiewicz, and E.S. Knudsen, CDK4/6 inhibitors have potent activity in combination with pathway selective therapeutic agents in models of pancreatic cancer. Oncotarget, 2014. 5(15): p. 6512-6525.

188. Bhat-Nakshatri, P., C.P. Goswami, S. Badve, L. Magnani, M. Lupien, and H. Nakshatri, Molecular Insights of Pathways Resulting from Two Common 
PIK3CA Mutations in Breast Cancer. Cancer Research, 2016. 76(13): p. 3989-4001.

189. Barbareschi, M., F. Buttitta, L. Felicioni, S. Cotrupi, F. Barassi, M. Del Grammastro, A. Ferro, P.D. Palma, E. Galligioni, and A. Marchetti, Different prognostic roles of mutations in the helical and kinase domains of the PIK3CA gene in breast carcinomas. Clinical Cancer Research, 2007. 13(20): p. 60646069.

190. Montaudon, E., J. Nikitorowicz-Buniak, L. Sourd, L. Morisset, R. El Botty, L. Huguet, A. Dahmani, P. Painsec, F. Nemati, S. Vacher, W. Chemlali, J. Masliah-Planchon, S. Chateau-Joubert, C. Rega, M.F. Leal, N. Simigdala, S. Pancholi, R. Ribas, A. Nicolas, D. Meseure, A. Vincent-Salomon, C. Reyes, A. Rapinat, D. Gentien, T. Larcher, M. Bohec, S. Baulande, V. Bernard, D. Decaudin, F. Coussy, M. Le Romancer, G. Dutertre, Z. Tariq, P. Cottu, K. Driouch, I. Bieche, L.A. Martin, and E. Marangoni, PLK1 inhibition exhibits strong anti-tumoral activity in CCND1-driven breast cancer metastases with acquired palbociclib resistance. Nature Communications, 2020. 11(1).

191. Lu, P.F., K. Takai, V.M. Weaver, and Z. Werb, Extracellular Matrix Degradation and Remodeling in Development and Disease. Cold Spring Harbor Perspectives in Biology, 2011. 3(12).

192. Barker, K.T., J.E. Martindale, P.J. Mitchell, T. Kamalati, M.J. Page, D.J. Phippard, T.C. Dale, B.A. Gusterson, and M.R. Crompton, Expression Patterns of the Novel Receptor-Like Tyrosine Kinase, Ddr, in Human BreastTumors. Oncogene, 1995. 10(3): p. 569-575. 
193. Neuhaus, B., S. Buhren, B. Bock, F. Alves, W.F. Vogel, and F. Kiefer, Migration inhibition of mammary epithelial cells by Syk is blocked in the presence of DDR1 receptors. Cellular and Molecular Life Sciences, 2011. 68(22): p. 3757-3770.

194. Turashvili, G., J. Bouchal, K. Baumforth, W. Wei, M. Dziechciarkova, J. Ehrmann, J. Klein, E. Fridman, J. Skarda, J. Srovnal, M. Hajduch, P. Murray, and Z. Kolar, Novel markers for differentiation of lobular and ductal invasive breast carcinomas by laser microdissection and microarray analysis. Bmc Cancer, 2007. 7.

195. Ding, L., G. Getz, D.A. Wheeler, E.R. Mardis, M.D. McLellan, K. Cibulskis, C. Sougnez, H. Greulich, D.M. Muzny, M.B. Morgan, L. Fulton, R.S. Fulton, Q.Y. Zhang, M.C. Wendl, M.S. Lawrence, D.E. Larson, K. Chen, D.J. Dooling, A. Sabo, A.C. Hawes, H. Shen, S.N. Jhangiani, L.R. Lewis, O. Hall, Y.M. Zhu, T. Mathew, Y.R. Ren, J.Q. Yao, S.E. Scherer, K. Clerc, G.A. Metcalf, B. Ng, A. Milosavljevic, M.L. Gonzalez-Garay, J.R. Osborne, R. Meyer, X.Q. Shi, Y.Z. Tang, D.C. Koboldt, L. Lin, R. Abbott, T.L. Miner, C. Pohl, G. Fewell, C. Haipek, H. Schmidt, B.H. Dunford-Shore, A. Kraja, S.D. Crosby, C.S. Sawyer, T. Vickery, S. Sander, J. Robinson, W. Winckler, J. Baldwin, L.R. Chirieac, A. Dutt, T. Fennell, M. Hanna, B.E. Johnson, R.C. Onofrio, R.K. Thomas, G. Tonon, B.A. Weir, X.J. Zhao, L. Ziaugra, M.C. Zody, T. Giordano, M.B. Orringer, J.A. Roth, M.R. Spitz, I.I. Wistuba, B. Ozenberger, P.J. Good, A.C. Chang, D.G. Beer, M.A. Watson, M. Ladanyi, S. Broderick, A. Yoshizawa, W.D. Travis, W.Pao, M.A. Province, G.M. Weinstock, H.E. Varmus, S.B. 
Gabriel, E.S. Lander, R.A. Gibbs, M. Meyerson, and R.K. Wilson, Somatic mutations affect key pathways in lung adenocarcinoma. Nature, 2008. 455(7216): p. 1069-1075.

196. Tomasson, M.H., Z. Xiang, R. Walgren, Y. Zhao, Y. Kasai, T. Miner, R.E. Ries, O. Lubman, D.H. Fremont, M.D. McLellan, J.E. Payton, P. Westervelt, J.F. DiPersio, D.C. Link, M.J. Walter, T.A. Graubert, M. Watson, J. Baty, S. Heath, W.D. Shannon, R. Nagarajan, C.D. Bloomfield, E.R. Mardis, R.K. Wilson, and T.J. Ley, Somatic mutations and germline sequence variants in the expressed tyrosine kinase genes of patients with de novo acute myeloid leukemia. Blood, 2008. 111(9): p. 4797-4808.

197. Fu, H.L., R.R. Valiathan, L. Payne, M. Kumarasiri, K.V. Mahasenan, S. Mobashery, P. Huang, and R. Fridman, Glycosylation at Asn211 Regulates the Activation State of the Discoidin Domain Receptor 1 (DDR1). Journal of Biological Chemistry, 2014. 289(13): p. 9275-9287.

198. Griffith, O.L., N.C. Spies, M. Anurag, M. Griffith, J. Luo, D. Tu, B. Yeo, J. Kunisaki, C.A. Miller, K. Krysiak, J. Hundal, B.J. Ainscough, Z.L. Skidmore, K. Campbell, R. Kumar, C. Fronick, L. Cook, J.E. Snider, S. Davies, S.M. Kavuri, E.C. Chang, V. Magrini, D.E. Larson, R.S. Fulton, S. Liu, S. Leung, D. Voduc, R. Bose, M. Dowsett, R.K. Wilson, T.O. Nielsen, E.R. Mardis, and M.J. Ellis, Author Correction: The prognostic effects of somatic mutations in ER-positive breast cancer. Nat Commun, 2018. 9(1): p. 4850.

199. Cerami, E., J.J. Gao, U. Dogrusoz, B.E. Gross, S.O. Sumer, B.A. Aksoy, A. Jacobsen, C.J. Byrne, M.L. Heuer, E. Larsson, Y. Antipin, B. Reva, A.P. 
Goldberg, C. Sander, and N. Schultz, The cBio Cancer Genomics Portal: An Open Platform for Exploring Multidimensional Cancer Genomics Data. Cancer Discovery, 2012. 2(5): p. 401-404.

200. Gao, J.J., B.A. Aksoy, U. Dogrusoz, G. Dresdner, B. Gross, S.O. Sumer, Y.C. Sun, A. Jacobsen, R. Sinha, E. Larsson, E. Cerami, C. Sander, and N. Schultz, Integrative Analysis of Complex Cancer Genomics and Clinical Profiles Using the cBioPortal. Science Signaling, 2013. 6(269).

201. Zeller, K.I., X.D. Zhao, C.W.H. Lee, K.P. Chiu, F. Yao, J.T. Yustein, H.S. Ooi, Y.L. Orlov, A. Shahab, H.C. Yong, Y.T. Fu, Z.P. Weng, V.A. Kuznetsov, W.K. Sung, Y.J. Ruan, C.V. Dang, and C.L. Wei, Global mapping of C-Myc binding sites and target gene networks in human B cells. Proceedings of the National Academy of Sciences of the United States of America, 2006. 103(47): p. 17834-17839.

202. Santoni-Rugiu, E., J. Falck, N. Mailand, J. Bartek, and J. Lukas, Involvement of Myc activity in a G(1)/S-promoting mechanism parallel to the $p R b / E 2 F$ pathway. Mol Cell Biol, 2000. 20(10): p. 3497-509.

203. Lanceta, L., C. O'Neill, N. Lypova, X.H. Li, E. Rouchka, S. Waigel, J.G. Gomez-Gutierrez, J. Chesney, and Y. Imbert-Fernandez, Transcriptomic Profiling Identifies Differentially Expressed Genes in Palbociclib-Resistant ER+MCF7 Breast Cancer Cells. Genes, 2020. 11(4).

204. Jeong, S.B., J.H. Im, J.H. Yoon, Q.T. Bui, S.C. Lim, J.M. Song, Y. Shim, J. Yun, J. Hong, and K.W. Kang, Essential Role of Polo-like Kinase 1 (Plk1) 
Oncogene in Tumor Growth and Metastasis of Tamoxifen-Resistant Breast Cancer. Molecular Cancer Therapeutics, 2018. 17(4): p. 825-837.

205. Liang, J., J. Zubovitz, T. Petrocelli, R. Kotchetkov, M.K. Connor, K. Han, J.H. Lee, S. Ciarallo, C. Catzavelos, R. Beniston, E. Franssen, and J.M. Slingerland, PKB/Akt phosphorylates p27, impairs nuclear import of p27 and opposes p27-mediated G1 arrest. Nat Med, 2002. 8(10): p. 1153-60.

206. Rossig, L., A.S. Jadidi, C. Urbich, C. Badorff, A.M. Zeiher, and S. Dimmeler, Akt-dependent phosphorylation of p21(Cip1) regulates PCNA binding and proliferation of endothelial cells. Mol Cell Biol, 2001. 21(16): p. 5644-57.

207. Das, S., P.P. Ongusaha, Y.S. Yang, J.M. Park, S.A. Aaronson, and S.W. Lee, Discoidin domain receptor 1 receptor tyrosine kinase induces cyclooxygenase-2 and promotes chemoresistance through nuclear factorkappa B pathway activation. Cancer Research, 2006. 66(16): p. 8123-8130.

208. Villalba, M., E. Redin, F. Exposito, M.J. Pajares, C. Sainz, D. Hervas, E. Guruceaga, A. Diaz-Lagares, C. Cirauqui, M. Redrado, K. Valencia, C. de Andrea, E. Jantus-Lewintre, C. Camps, R. Lopez-Lopez, A. Lahoz, L. Montuenga, R. Pio, J. Sandoval, and A. Calvo, Identification of a novel synthetic lethal vulnerability in non-small cell lung cancer by co-targeting TMPRSS4 and DDR1. Sci Rep, 2019. 9(1): p. 15400.

209. Cristofanilli, M., A. DeMichele, C. Giorgetti, N.C. Turner, D.J. Slamon, S.A. Im, N. Masuda, S. Verma, S. Loi, M. Colleoni, K. Puyana Theall, X. Huang, Y. Liu, and C. Huang Bartlett, Predictors of prolonged benefit from palbociclib plus fulvestrant in women with endocrine-resistant hormone receptor- 
positive/human epidermal growth factor receptor 2-negative metastatic breast cancer in PALOMA-3. European Journal of Cancer, 2018. 104: p. 21-31.

210. O'Leary, B., R.J. Cutts, Y. Liu, S. Hrebien, X. Huang, K. Fenwick, F. Andre, S. Loibl, S. Loi, I. Garcia-Murillas, M. Cristofanilli, C.H. Bartlett, and N.C. Turner, The Genetic Landscape and Clonal Evolution of Breast Cancer Resistance to Palbociclib plus Fulvestrant in the PALOMA-3 Trial. Cancer Discovery, 2018. 8(11): p. 1390-1403.

211. Gordus, A., J.A. Krall, E.M. Beyer, A. Kaushansky, A. Wolf-Yadlin, M. Sevecka, B.H. Chang, J. Rush, and G. MacBeath, Linear combinations of docking affinities explain quantitative differences in RTK signaling. Mol Syst Biol, 2009. 5: p. 235.

212. Vehlow, A., E. Klapproth, S. Jin, R. Hannen, M. Hauswald, J.W. Bartsch, C. Nimsky, A. Temme, B. Leitinger, and N. Cordes, Interaction of Discoidin Domain Receptor 1 with a 14-3-3-Beclin-1-Akt1 Complex Modulates Glioblastoma Therapy Sensitivity. Cell Rep, 2019. 26(13): p. 3672-3683 e7.

213. Hinds, P.W. and R.A. Weinberg, Tumor-Suppressor Genes. Current Opinion in Genetics \& Development, 1994. 4(1): p. 135-141.

214. Brennan, P., J.W. Babbage, B.M.T. Burgering, B. Groner, K. Reif, and D.A. Cantrell, Phosphatidylinositol 3-kinase couples the interleukin-2 receptor to the cell cycle regulator E2F. Immunity, 1997. 7(5): p. 679-689.

215. O'Leary, B., R.J. Cutts, Y. Liu, S. Hrebien, X. Huang, K. Fenwick, F. Andre, S. Loibl, S. Loi, I. Garcia-Murillas, M. Cristofanilli, C. Huang Bartlett, and N.C. Turner, The Genetic Landscape and Clonal Evolution of Breast Cancer 
Resistance to Palbociclib plus Fulvestrant in the PALOMA-3 Trial. Cancer Discov, 2018. 8(11): p. 1390-1403. 


\section{VITA}

Maryam Shariati the daughter of Nasrin and Mohammad Hassan Shariati, was born in Qom, Iran. She entered the University of California, Irvine where she received the degree of Bachelor of Science with a major in cell and developmental biology. She then received the degree of Master of Science from the University of Texas MD Anderson Cancer Center UTHealth, Graduate School of Biomedical Sciences and proceeded to PhD program.

Permanent address:

9009 Memorial Dr.

Houston, Texas 77024 